Programa de Doctorado en Ingeniería Matemática, Estadística e Investigación Operativa por la

Universidad Complutense de Madrid y la

Universidad Politécnica de Madrid
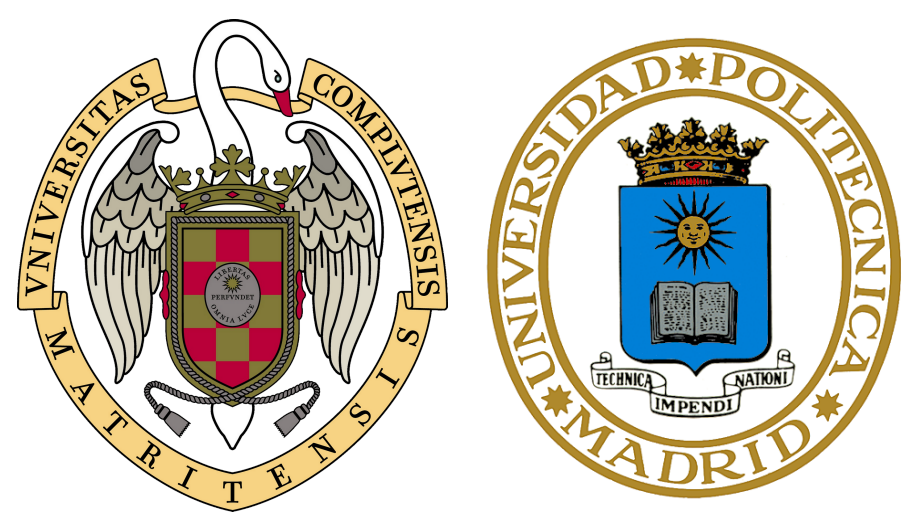

\title{
Integrable Lagrangian Systems and Symmetries
}

\author{
Tesis Doctoral \\ Agustín Caparrós Quintero \\ Director \\ Rafael Hernández Heredero
}

Año

2018 

PhD Thesis

INTEGRABLE LAGRANGIAN SYSTEMS AND SYMMETRIES AGUSTÍN CAPARRÓS QUINTERO

Departamento de Matemática Aplicada a las Tecnologías de la Información y las Comunicaciones

E.T.S.I.S. Telecomunicación

Universidad Politécnica de Madrid

September 2018 

Tribunal nombrado por el Mgfco. y Excemo. Sr. Rector de la Universidad Politécnica de Madrid, el día de de

$\begin{array}{ll}\text { Presidente } & \text { D. } \\ \text { Vocal } & \text { D. } \\ \text { Vocal } & \text { D. } \\ \text { Vocal } & \text { D. }\end{array}$

Vocal Secretario D.

Realizado el acto de defensa y lectura de la Tesis el día de de , en

Calificación: 

This thesis has been written by Agustín Caparrós Quintero to acquire the PhD degree Doctor por la Universidad Complutense de Madrid y la Universidad Politécnica de Madrid, after completion of all academic activities under the IMEIO doctoral program (http://www. mat. ucm. es/ imeio/). It is based on the personal research of the student under guidance of supervisor Rafael Hernández Heredero on the subject of Lagrangian systems integrability, a topic in Mathematical Physics.

After introducing the symmetry approach to integrability, an extended formal symmetry approach is used to obtain new results in second order integrable Lagrangian systems. As an application, a classification of such systems is done and described in full detail. All the computational techniques used are detailed as well as the exact functional expressions of the integrable classes representatives.

This thesis focuses on the extension to higher orders of the symmetry approach to the integrability of Partial Differential Equation (PDE)s. The extended symmetry approach is applied to non-evolutionary equations arising as Euler-Lagrange equations of second order Lagrangian systems, whose integrability conditions are explicitly computed and applied to the classification of their integrable cases. The theory underlying the symmetry approach and its extension, as described in part I, is that arising from Lie groups, differential invariance and symmetries applied to systems of differential equations. Although a more specific theoretical framework exists for the determination of symmetries of variational problems, for the purposes of this Thesis it is enough to use the general theory for differential equations, disregarding the variational structure.

In the second part of this thesis we detail the computational techniques employed to derive all cases and expressions in the integrable Lagrangian systems classification. In chapter 5 , the classification results are presented and some of them are checked to comply with the theoretical formulation of the extended symmetry approach for equations of generic order $(n, m)$. In order to achieve this, some explicit, non-formal recursion operators are computed and tables of explicit canonical densities are provided. For instance, special cases of second order Lagrangian systems associated with the well-known Boussinesq equation or Non Linear Schrodinger (NLS) equations are studied in detail. The classification also includes a number of seemingly new cases of integrable Lagrangians. 

Esta tesis ha sido escrita por Agustín Caparrós Quintero para optar al grado de Doctor por la Universidad Complutense de Madrid y la Universidad Politécnica de Madrid, tras la finalización de todas las actividades académicas comprendidas el programa doctoral IMEIO (http://www. mat . ucm.es/imeio/). Está basada en la investigación, bajo la supervisión del Dr. Rafael Hernández Heredero, en integrabilidad de sistemas Lagrangianos, un tópico de la Física Matemática.

Tras presentar el método de las simetrías formales para la clasificación de ecuaciones integrables, utilizamos su extensión a ecuaciones no evolutivas para obtener nuevos resultados en el campo de los sistemas lagrangianos integrables. Como aplicación, se realiza una clasificación de dichos sistemas de segundo orden y se describe todo el proceso seguido hasta la obtención de las expresiones funcionales exactas para los representantes de las clases encontradas.

En esta tesis nos centramos en la extensión a ordenes superiores del método de las simetrías formales para la integrabilidad de ecuaciones diferenciales en derivadas parciales. El método extendido se aplica a ecuaciones no evolutivas que aparecen como ecuaciones de Euler-Lagrange de sistemas lagrangianos de segundo orden, cuyas condiciones de integrabilidad son calculadas explícitamente y aplicadas a la clasificación de sus casos integrables. Los fundamentos teóricos del método de simetrías formales y de sus extensiones, tal y como se describen en la primera parte, provienen de la teoría de grupos de Lie, invariantes diferenciales y simetrías aplicados a sistemas de ecuaciones diferenciales. Aunque existe un marco teórico más especifico para la determinación de simetrías de problemas variacionales, para los objetivos de esta tesis es suficiente el uso de la teoría general para ecuaciones diferenciales, sin tener en cuenta la estructura variacional.

En la segunda parte detallamos las técnicas de cálculo empleadas para la clasificación de sistemas lagrangianos integrables de segundo orden. En el capítulo 5, se presentan los resultados de la clasificación y para algunos de ellos se comprueban contra los fundamentos teóricos del método extendido de simetrías formales para ecuaciones de orden genérico $(n, m)$. Para ello se calculan algunos operadores de recursión en forma explícita para sistemas de tipos bien conocidos como Boussinesq o NLS. La clasificación contiene también algunos casos de sistemas lagrangianos integrables que podrían ser nuevos. 



\section{PUBLICATIONS}

Some concepts, propositions and theorems have appeared previously in the following publications:

[39] A. Caparrós Quintero, R. Hernández Heredero. “Formal recursion operators of integrable nonevolutionary equations and Lagrangian systems". J. Phys. A: Math. Theor. 51 (2018) 385201 (22pp)

https://doi.org/10.1088/1751-8121/aad63e 

We have seen that computer programming is an art, because it applies accumulated knowledge to the world, because it requires skill and ingenuity, and especially because it produces objects of beauty.

- Donald E. Knuth -

\section{ACKNOWLEDGMENTS}

Many thanks to all who in a form or another helped in making this personal project successful.

Especially to my PhD advisor and supervisor Rafael Hernández Heredero for his continuous example in showing me how Mathematics are done at the research level. I am also grateful to Juan José Morales-Ruiz and everyone in the research group of integrable systems at Madrid Technical University (ETSI Caminos Canales y Puertos and ETS Edificación) for the same reasons and for their kindness when welcoming me in their seminaries.

The academic research activities for this work were partially supported by Ministerio de Economía y Competitividad to the project "Quantum groups, Poisson-Lie symmetries and integrable systems" at University of Burgos, Spain, under grant number MTM2013-43820-P. 


\title{
CONTENTS
}

\author{
Introduction $\quad$ ix
}

I SYMMETRIES AND INTEGRABILITY

1 FROM LIE TO FORMAL SYMMETRIES 3

1.1 Classical symmetries and symmetry condition 4

1.1.1 Infinitesimal invariance. 4

1.1.2 Lie symmetries of a system of differential equations. 6

1.1.3 Characteristics and Evolutionary form of symmetries 9

1.2 Generalized symmetries and recursion operators 10

1.3 Evolutionary vector fields and symmetries II

1.4 Symmetries of evolution equations 13

1.5 Integrability types and definitions 14

1.6 Formal symmetries and integrability of evolution equations 16

1.7 Formal symmetries and formal recursion operators 18

1.8 Integrability conditions 21

1.9 Conservation laws and conserved densities 22

1.10 Canonical conservation laws as integrability conditions 23

1.11 Classification method 25

1.12 Second order evolution equations 26

1.12.1 Equations of the form $u_{t}=u_{x x}+K\left[x, u, u_{x}\right] \quad 26$

1.12.2 Sketch of the formal symmetry method applied to the classification of second order integrable equations. $\quad 28$

1.12.3 Classification theorem 30

1.13 Higher order evolution equations 30

1.14 Jet Calculus and Symmetries 31

2 EXTENSIONS TO THE FORMAL SYMMETRY APPROACH 37

2.1 State of the art of the symmetry approach to integrability 37

2.2 Extensions to non-evolutionary equations 38

2.3 The structure of the solution space 41

2.4 Extended symmetry approach and Lagrangian systems classification. 51

3 Allowed differential substitutions 55

3.1 Point transformations 55

3.2 Contact transformations and contact condition 56

3.3 Method for the construction of contact transformations 57

3.4 Contact transformations for evolution and non evolutionary equations $\quad 58$

3.5 Change of coordinates in the Lagrangian systems classification 60

4 COMputer algebra algorithms 61

4.1 Symbolic vector calculus functions 61

4.2 CCL integration technique 62

4.3 Obstructions analysis 66

4.4 Proofs of integrability 68 
II RESULTS AND OPEN PROBLEMS

5 SECOND ORDER INTEGRABLE LAGRANGIAN SYSTEMS CLASSIFICATION 71

5.1 Initial conditions 71

5.2 Integrability conditions from canonical conservation laws $\quad 72$

5.3 Case $\mathrm{A}: K_{020}=0, L_{021}(u) \neq 0 . \quad 76$

5.3.1 Case A-1: $L_{200 A}(u)=0 . \quad 77$

5.3.2 Case A-2: $L_{200 A}(u) \neq 0 . \quad 81$

5.4 Case B: $K_{020} \neq 0, L_{021}(u)=0 . \quad 83$

5.4.1 Case B-1: $K_{202}:=0 . \quad 84$

5.4.2 Case B-2: $K_{202} \neq 0 . \quad 87$

5.5 Equivalent Lagrangians under point transformations 90

5.5.1 Equivalent form of cases representatives 90

5.5.2 Equivalence between cases representatives 95

5.6 Listing of non-equivalent integrable cases 95

5.7 Additional considerations 98

6 RECURSION OPERATORS 103

6.1 Determining equations for formal recursion operators of non-evolutionary equations. $\quad 103$

6.2 Recursion operators of integrable Lagrangian systems 107

6.2.1 Linear Lagrangian $\mathcal{L} 1 \quad 107$

6.2.2 Lagrangian $\mathcal{L} 2 \quad 107$

6.2.3 Lagrangian $\mathcal{L} 3 \quad 108$

6.2.4 Lagrangian $\mathcal{L} 3$ (matrix form) $\quad 109$

6.2.5 Lagrangian $\mathcal{L} 4 \quad 109$

6.2.6 Lagrangian $\mathcal{L} 5 \quad 111$

6.2.7 Lagrangian $\mathcal{L} 6 \quad 111$

6.2.8 Lagrangian $\mathcal{L} 7 \quad 111$

6.2.9 Lagrangian $\mathcal{L} 8 \quad 112$

7 CONCLUSIONS AND OPEN PROBLEMS 115

7.1 Conclusions 115

7.2 Open problems 116

III APPENDIX

CANONICAL DENSITIES 119

BIBLIOGRAPHY 119 


\section{ACRONYMS}

ODE Ordinary Differential Equation

PDE Partial Differential Equation

FRO Formal Recursion Operator

KdV Korteweg-de-Vries equation

mKdV modified Korteweg-de-Vries equation

CCL Canonical Conservation Law

IEEC Integrable Evolution Equation Classification

ILSC Integrable Lagrangian Systems Classification

NLS Non Linear Schrodinger

\section{LIST OF FIGURES}

Figure 4.2.1 Computations flow for Integrable Evolution Equation Classification (IEEC) 63

Figure 4.2.2 Computations flow for Integrable Lagrangian Systems Classification (ILSC) $\quad 65$

\section{LISTINGS}

Listing 4.1 Total derivatives functions and verification of conservation law for modified Korteweg-de-Vries equation (mKdV). 62

Listing 4.2 Sample of CCL Integration template code 66

Listing 4.3 Sample screenshot 67

Listing 4.4 Sample of code template for proof of integrability 68 
LIST OF TABLES

Table 2.1

Table 5.1

Table 5.2

Table $5 \cdot 3$

Table 5.4

Table $5 \cdot 5$

Table 5.6

Table $5 \cdot 7$

Table 5.8
Table of conserved densities of equations of the form $u_{t t}=H\left(u_{x x x x}\right.$, $\left.u_{x x x}, u_{x t}, u_{x x}, u_{t}, u_{x}, u\right)$

Lagrangian densities after point transformations, 1 of $4 \quad 91$

Lagrangian densities after point transformations, 2 of $4 \quad 92$ Lagrangian densities after point transformations, 3 of 493 Lagrangian densities after point transformations, 4 of $4 \quad 94$ Equivalence between case representatives $\quad 96$ Final integrable cases $\quad 97$ Second order integrable Lagrangian systems class representatives (up to $\left.\rho_{6}\right) \quad 97$

Second order integrable Lagrangian systems class representatives (up to $\left.\rho_{10}\right) \quad 101$ 
This thesis deals with certain integrable partial differential equations. Integrability is a word that refers to different notions in Mathematics. As a general concept, it can be associated to the solvability of a mathematical problem or equation. With this interpretation, integrability resides at the very core of Mathematics since its inceptions. The problem of solving quadratic or cubic equations, or rather, of which quadratic or cubic equations could be solved by the methods of the time, appeared already in the Babylonian civilization [5]. Greek mathematics were profoundly influenced by the problem of the solvability of equations, up to the point of discovering the irrational numbers and setting the "quadrature of the circle" problem, which is certainly a problem in integrability: is a square of area equal to that of a given circle constructible with ruler and compass?. The problems of solvability of low order polynomial equations in terms of their coefficients continued to have a central stage in Medieval mathematics, as we can see in the classical treaty "Kitab al-Jabr" of al-Khwarizmi. Tartaglia, Cardano, Ferrari, Vieta are further examples of such search for solvability of polynomial equations of order up to four. After the solution of Ferrari/Vieta to the general fourth order equation was published (1545-1615), a long period passed by with many powerful mathematicians trying to solve, unsuccessfully, the quintic equation. In 1771 Lagrange [22] made a deep study of all the previous results on polynomial equations and conjectured that solving the quintic equation (by radicals on its coefficients) could not be possible by the methods known at the time. Gauss was perhaps the first one to understand that it would be better to try proving that the problem was not solvable, having the first glimpse of actual non-integrability. AbelRuffini's theorem in 1824 finally proved the unsolvability of the problem. It was of course Galois who discovered the relation of the theory of equations with group theory, providing deepest understanding on the problem of solvability of polynomial equations.

What about differential equations? It seems that the word integral was in fact used for the first time by Johann Bernouilli in a letter to Leibniz [10, p. 107], and that the Bernouillis used "to integrate" meaning solving a differential equation finding the quadrature of a given integral. Thus, the word integrable can be used to qualify a differential equation that can be solved in quadratures. But of course this idea must be elaborated specifying what class of functions can be used to describe the quadratures or the solutions themselves. It was probably Liouville, who also published the works of Galois, the first mathematician to advance in this direction, providing his the- 
orem on the "integrability" of functions in finite terms (loosely algebraic functions, exponential, logarithms). This was the beginning of a fruitful theory of integrability of ordinary differential equations understood as solvability in specific classes of functions in terms of the coefficients of the equation, namely differential Galois theory. It includes the Picard-Vessiot theory for linear ODEs, Kolchin's results, Kovacic's theorem, the Morales-Ramis theorem, and it is presently being actively developed, struggling and starting to be extended into the PDE's realm.

In another direction we have the concept of Liouville integrability of a Hamiltonian system of ODEs [24]. It states that finding explicitly a number of compatible first integrals of such a system equal to the number of its degrees of freedom (half the number of dependent variables), one can deem the system as "integrable". This statement does not directly refer anymore to solvability, unless the nature of the first integrals is specified.

In the field of PDEs, several concepts of integrability have appeared, all much weaker than that of Galoisian integrability. The word integrability in the field of PDEs already appears in Frobenius theorem (1877) on the "integrability" of involutive systems of first order PDEs [13]. Here integrability means the existence of maximal integral surfaces. In this line, great contributions have been made by mathematicians like E.Cartan, Kuranishi, Spencer, etc. Another concept of integrability is formal integrability (Goldschmidt) which is basically an existence theory generalizing the classical Cartan-Kähler theorem on the local existence of solutions to overdetermined systems of PDEs. Of course, Sophus Lie's developed his symmetry analysis to achieve in differential equations what Galois had done with polynomial equations, a solvability theory. We will speak about this below.

Our approach to integrability is that of the so-called symmetry approach to integrability. It was developed by A.B. Shabat [45] and collaborators, starting in the 1970s. It is applicable to ODEs and directly applicable to PDEs, and it admits an algorithmic, constructive setting. It could be said that it lies at an intermediate state between existence-compatibility theories mentioned above, and a hypothetical Galoisian solvability theory of PDEs, being in the line, as we will see, of a "Liouville integrability" theory of infinite dimensional dynamical (Hamiltonian or not) systems.

The triggering event that provoked the modern development of the integrability theory of PDEs was the discovery, in 1967 by Gardner et al. [14], of the inverse scattering transform method to solve the Korteweg-de-Vries equation (KdV). The KdV equation [21] is an evolution partial differential equation that models a $1+1$ hydrodynamic system: non-linear waves in a shallow and narrow long channel. We write is as

$$
u_{t}=u_{x x x}+6 u u_{x}
$$


where $u=u(x, t)$ and subscripts mean partial differentiation. The conventionally accepted first person to observe and study a nonlinear wave was J. Scott Russell [41], who reported observing (and subsequently analyzing in the laboratory) a "solitary wave" in a Scottish channel. This wave was proved by Korteweg and de Vries to be a special solution of KdV. The next chapter in the KdV story is perhaps the numerical experiment that Fermi, Pasta, Ulam and Tsingou [11] performed in 1955 to clarify why energy is distributed evenly between all normal modes in a real oscillating system. With the first electronic computers available, they could simulate a discrete oscillating linear chain with a first corrective non-linear term, and observed with surprise how, instead of the energy being "thermalized", some seemingly periodic recurrence arose in the system. Zabusky and Kruskal performed a continuous limit of this non-linear chain that lead to the KdV equation in [50], and coined the term "soliton", being Russell's solitary wave the simplest one. Solitons are non-linear localized waves that asymptotically do not interact when colliding, explaining thus the non-thermalization paradox. Attempts to understand this rigid behaviour lead to the "discovery" of many conservation laws of $\mathrm{KdV}$, eventually infinite [30] and, being these objects the PDE's counterpart of ODE's first integrals, a first glimpse of an integrability theory for KdV appeared. Immediately, P. Lax [23] was able to write $\mathrm{KdV}$ as the compatibility condition of two linear equations, being one a spectral problem, and showing that the $\mathrm{KdV}$ evolution in $t$ was an isospectral evolution. This pair of equations is the celebrated Lax pair. This formulation would have been only a happy coincidence if it were not for the discovery, in a very short time, of Zakharov and Shabat [51] stating that another well known peculiar equation, the non-linear Schrödinger equation, was also amenable to be written in Lax pair form, and thus in priciple "integrable" via the inverse scattering theory. Ablowitz, Kaup, Newel and Segur generalized the Zakharov-Shabat method and found that other remarkable differential equations known from the nineteenth century, like the modified $\mathrm{KdV}$ equation or the sine-Gordon equation, were amenable to be written as Lax pairs. These equations were known to possess impressive (but perhaps a bit forgotten by then) geometrical and symmetry properties, like the so-called Bäcklund transformations (Bäcklund [3] and Bianchi [6]) and the rediscovered Miura transformation [28]. All these discoveries called the attention of mathematical physicists and lead to the present corpus of results that is "soliton theory" and the theory of integrable solitonic equations. These theories are far to be complete, and a constant flow of new results, including those in this thesis, keeps going steadily.

But, what has symmetry to do about integrable equations? Many objects mentioned above are related to symmetries of different types. The Lax isospectral evolution possesses a series of invariants (related 
to the invariant eigenvalues) that can be regarded as invariants of certain sophisticated symmetry transformation. Integrable equations are invariant under so-called auto-Bäcklund transformations. The classical symmetry analysis pervades the scattering inverse theory [29]. As we said before, Lie himself was inspired by Galois theory to develop his symmetry theory on differential equations, trying to find ways to solve them. In [2] a discussion of how many classical solution procedures for ODEs, such as separation of variables, homogeneity, etc., can be explained by the admittance of Lie symmetries by the equation. As explained in Olver [35, p. xviii], after Lie, other researchers and the important contributions of E. Noether, the huge advances of E. Cartan during the 1930's in group theory made the interest on Lie symmetry analysis of differential equations to fade during a long period. Nevertheless, practical fields like hydrodynamics, elasticity, etc. saw many applications of the related dimensional analysis (Barenblatt [4] and Taylor [49]). Always according to Olver, the first one to recover interest in the west, during the 1950's, was G. Birkhoff [7] and in the Soviet Union L. V. Ovsiannikov (cf. Ovsiannikov [38]) who created an important school in Novosibirsk, being one of its members A. B. Shabat, the creator of the symmetry approach to integrability. Lie theory maintained, though, its importance in Physics, especially in Nuclear and Field Theory Physics.

Classical Lie symmetries of an evolution equation with two independent variables $t, x$ and one dependent variable $u$

$$
u_{t}=f\left(t, x, u, u_{x}, \ldots, u_{n}\right), \quad u_{n}:=\partial^{n} u / \partial u^{n}
$$

have as infinitesimal generators vector fields

$$
\mathbf{v}=\tau(t, x, u) \partial_{t}+\xi(t, x, u) \partial_{x}+\phi(t, x, u) \partial_{u} .
$$

This type of symmetries encompasses all the dimensional analysis theory, hodograph transformation theory, etc. Already Noether realized that in her theorem about the relation between conservation laws and symmetries of Lagrangian systems, the symmetries could be of a more general form, the so called generalized symmetries. Generalized symmetries have generators

$$
\begin{aligned}
\mathbf{v}=\tau\left(t, x, u, u_{x}, \ldots, u_{m}\right) \partial_{t}+\xi\left(t, x, u, u_{x}, \ldots, u_{m}\right) \partial_{x} \\
\\
+\phi\left(t, x, u, u_{x}, \ldots, u_{m}\right) \partial_{u}
\end{aligned}
$$

whose coefficients depend not only on the configurational variables $t$, $x, u$ but on derivatives of $u$. Predecessors of generalized symmetries were contact Lie symmetries, already studied by Lie, with coefficients depending up to the first derivative $u_{x}$ and satisfying an additional "contact condition" that restricts their action to the first jet, i.e. the space of variables $t, x, u, u_{x}$. As we will see, the action of more general generalized symmetries, as a theorem by Bäcklund proves [3], 
is not restricted to a finite order jet, but inevitably prolongs to the infinite order jet (cf. eq. 1.1.6). Linear equations admit this type of symmetries, but very few nonlinear equations do. In fact, all solitonic integrable equations admit generalized symmetries, and more precisely, an infinite hierarchy of them of arbitrarily high order $m$. Integrable equations, as be will explain in next chapter, can be characterized by the property of admitting such an infinite hierarchy of generalized symmetries. Going a little bit further in this observation, Olver defined [36] a recursion operator, i.e. an operator that maps a generalized symmetry into another generalized symmetry of higher order. These operators are admitted by linear equations and by solitonic integrable equations. So a further definition of an integrable equation is as that equation that admits a recursion operator, and a seed symmetry. In this sense, integrability based on symmetries states that nonlinear equations are integrable if they have the same symmetry structure than linear equations.

Recursion operators can be represented by pseudodifferential series

$$
\mathscr{R}=r_{m} D^{m}+r_{m-1} D^{m-1}+\cdots+r_{0}+r_{-1} D^{-1}+r_{-2} D^{-2}+\cdots
$$

on the total derivative $D:=D_{x}$. When $\mathscr{R}$ cannot be written in finite form, it is called formal recursion operator. Shabat's theory proposes to study in detail the equation characterizing formal recursion operators 1.6.7:

$$
\mathscr{R}_{t} \doteq\left[\mathrm{D}_{f}, \mathscr{R}\right]
$$

where $\mathrm{D}_{f}$ is the so-called Fréchet derivative of the r.h.s. $f$ of the evolution equation o.o.1. As we will see, this, interestingly, requires that certain recursively computable expressions in terms of the r.h.s. $f$ of the equation must be conservation laws, called canonical conservation laws. Admitting these canonical conservation laws is the condition of integrability for a given equation. The canonical conservation laws are algorithmically computable. For example, for second order evolution equations

$$
u_{t}=f\left(x, u, u_{x}, u_{x x}\right)
$$

the first two canonical densities are the expressions

$$
\rho_{0}=\left(\frac{\partial f}{\partial u_{x x}}\right)^{-1 / 2}, \quad \rho_{1}=\rho_{0}^{2} \frac{\partial f}{\partial u_{x}}-\rho_{0} \sigma_{0}
$$

and the canonical conservation laws are $D_{t} \rho_{0}=D_{x} \sigma_{0}$ and $D_{t} \rho_{1}=$ $D_{x} \sigma_{1}$. These equations restrict in such a high degree the form of $f$ that a classification of integrable equations is attainable. Just the first five canonical conservation laws allow to reach the classification given by Svinolupov [47] (cf. Theorem 9). A word of caution is necessary here: to be integrable according to our definition, the equation has to admit an infinite number of canonical conservation laws. In practice, 
just requiring a few integrability conditions reduces the integrable cases to a short list of classes of equations equivalent under contact transformations. Proving with an alternative procedure the integrability of each of the classes is enough to finish the classification. In the case of Theorem 9, Svinolupov proved that all the given classes are related, through a so called Cole-Hopf transformation, to the linear equation $u_{t}=u_{x x}+q(x) u$. It can be proved that the Cole-Hopf transformation preserves our concept of integrability and, thus, all the given equations are integrable.

The original formal symmetry approach was applicable only to scalar evolution equations o.o.1. Later, in Mikhailov, Shabat, and Yamilov [25], the theory was extended to matrix evolution equations. But there was the restriction that the matrix of the coefficients of the highest derivatives in the r.h.s should be diagonalizable and with different eigenvalues. In [16], an extension to nondiagonalizable systems was presented. This was applied to research a family of nonevolution equations of the form $u_{t t}=f\left(u, u_{x}, u_{t}, u_{x x}, u_{x t}, u_{x x x}\right)$. In this thesis, we have made an study of equations of the form $u_{t t}=$ $f\left(u, u_{x}, u_{t}, u_{x x}, u_{x t}, u_{x x x}, u_{x x x x}\right)$ arising from Lagrangian systems. In order to achieve it, it has been necessary to perform a considerable generalization to the theoretical results in [16]. This generalization has clarified what would be the general structure of the space of formal recursion operators and, consequently, of the integrability conditions of systems of a general form $u_{t \cdots t}=f\left(x, u, u_{x}, u_{t}, \ldots, u_{N}\right)$. The obtained list of eight integrable Lagrangian systems contains three known, physically significant, cases such us the nonlinear Schrödinger system, the Boussinesq equation and a Landau-Lifshitz magnetic system. Besides, some new systems have being obtained and their integrability has been proved in almost all cases by providing a nonformal recursion operator and a seed symmetry. 


\section{Part I}

SYMMETRIES AND INTEGRABILITY 

The starting point of our research is Lie symmetry analysis. The applications of Lie groups of infinitesimal transformations to the study of differential equations generalizes to the calculus methods that we use in search for new results in the subject of integrable Lagrangian systems. The underlying theory of our work can be formulated using the formalism of differential geometry, manifolds and Lie groups and extends to jet (or prolongation) spaces calculus.

A symmetry of a given equation is defined as any transformation of the space of variables preserving the condition that the equation imposes to its solutions, this is, mapping solutions to solutions. Lie's methods generate solutions from solutions by means of the knowledge of the equation's symmetries. This approach is the basis for both:

- The theoretical foundations of almost all the well known methods for integration of Ordinary Differential Equation (ODE)s. These methods set the procedure for solving a differential equation by suggesting the appropriate change of variables for each equation type (separable, homogeneous, exact, first integrals, etc.), and this suggestion is based in the symmetries of the given equation. These two (changes of variables and equation types) are key elements underlying the theory of integration, related to equivalence transformations and canonical form of equations.

- The study of systems of PDEs and therefore for a wide range of science and engineering problems. These including the generation of conservation laws and constants of motion, with applications in hydrodynamics, elasticity, scattering theory, relativity, stability, etc. The action of Lie groups on differential manifolds provide a systematic way of finding qualitative properties of solutions and, specially for non-linear equations, an analytic method for determining exact solutions, from other exact solutions. Solving integrability conditions found trough symmetry analysis leads in turn to determine equivalence classes of integrable equations, something that we pursue to extend to Lagrangian systems.

Noether's theorems for variational systems, stating that every conservation law comes from a symmetry, provide a method for constructing conservation laws of systems of differential equations. Given a variational structure in the system under consideration, this method represents the first generalization of the concept of geometric or Lie 
Symmetry theory generalizes integrability

foundations from ordinary to systems of partial differential equations and generic integrable systems, and as we shall see, as a central point of our research, to Lagrangian systems. symmetry by allowing the symmetry objects to depend not only on function's coordinates but also on the dependent variable's derivatives, prolonging the base manifolds from geometric to jet spaces.

Lie's and Noether's theory evolved in the past century towards the framework of formal symmetries, with the Lie derivative as the invariant cornerstone concept providing a key to the theory of integration of differential equations from all the algebraic, geometric and differential points of view. The Lie derivative and Lie bracket, reviewed in the next section, are the central concept of the theory from the purely geometric initial conception of a symmetry, to the jet calculus formal framework involving pseudo-differential algebra which is necessary to formalize the approach to determine the integrability of higher order PDEs.

In this introductory chapter we will explain the concepts and methods in classical symmetry theory and how they generalize to different types of symmetries from classical or geometrical symmetries, the socalled generalized symmetries, or the more subtle and powerful concept of formal symmetry. We base our exposition in standard references like Mikhailov, Shabat, and Sokolov [26] and Olver [34, 35]. We introduce the concepts and terminology necessary for understanding the techniques and results used in this thesis, along with specific notation and computational methods. In the second part, fully detailed calculations of the results are given.

\section{I CLASSICAL SYMMETRIES AND SYMMETRY CONDITION}

\subsubsection{Infinitesimal invariance.}

Symmetries in mathematics formalize the concept of patterns and invariance. The algebraic definition of a group systematizes and makes the symmetry concept computable. When applied to equations we find that the symmetries of a given system can be interpreted as the group of self-equivalences, and equivalence deals with the determination of when two mathematical objects are the same (can be transformed into each other) under a change of variables. Thus our road to classification begins by finding the symmetry group admitted by a system of equations and isomorphism of these groups will lead to equivalence and classification. Lie's classification of symmetry groups acting on one and two-dimensional manifolds can be found in Olver [35] representing the first example of the equivalence problem applied to differential systems classification. The sets, quantities or functions which are unaffected by changes of variables are the invariants, which for transformations groups acting on the space of the variables of equations are determined by the invariance or symmetry conditions. We will obtain several statements of these symmetry 
conditions for different types of symmetries and use them as starting point for our calculations.

The symmetry condition for algebraic equations can be explicitly found by determining the changes of coordinates $\bar{x}$ that make an equation $F\left(x_{1}, x_{2}, \ldots, x_{n}\right)=0$ invariant, which is noted $F(\bar{x}) \doteq 0$ meaning "on solutions to" $F(x)=0$, this is, leaving the subvariety determined by the common zeros or set of solutions, invariant:

$$
F\left(\overline{x_{1}}, \overline{x_{2}}, \ldots, \overline{x_{n}}\right)=0 \text { when } F\left(x_{1}, x_{2}, \ldots, x_{p}\right)=0
$$

The first observation about the group structure of the set of symmetries of a geometric object is due to Galois (see Ibragimov and Kruglikov [19] for the relation between the algebraic and analytic cases). Lie generalized this idea from algebra to analysis with the introduction of continuous groups of transformations acting geometrically on the space of independent and dependent variables, and bearing his name as an extension of Galois's theory to the study of differential equations. In both the algebraic and differential cases, invariants for systems of equations can be found using Lie's infinitesimal criterion of invariance, which replaces the non linear conditions of invariance of a subset or function under the group of transformations implicit in equation 1.1.1 by an equivalent linear, simpler, condition of infinitesimal invariance under the corresponding infinitesimal generators of a group of transformations action. In order to illustrate these concepts, in this chapter we are following Olver [34].

The invariant functions over a manifold $M, \zeta: M \rightarrow \mathbb{R}$ for a connected group $G$ are the solutions to

$$
\mathbf{v}(\zeta)=0
$$

for every $x \in M$ and every infinitesimal generator $\mathbf{v}$. Infinitesimal generators generate the action of $G$ just as one-parameter groups of transformations are generated as the flow of a vector field. They are tangent vector fields that form a Lie algebra $\mathfrak{g}$, meaning that local commuting flows have their commutator $[\mathbf{v}, \mathbf{w}]$ vanished and coinciding with the local action of $G$. Thus, a local invariant $\zeta(x)$ of the group of symmetries $G$ with infinitesimal generator $\mathbf{v}=\sum_{i} \xi^{i}(x) \frac{\partial}{\partial x^{i}}$ is a solution of the linear homogeneous first order partial differential equation

$$
\mathbf{v}(\zeta)=\sum_{i=1}^{p} \xi^{i}(x) \frac{\partial \zeta}{\partial x^{i}}=0 .
$$

The general solution to this equation can be found by integration of its characteristic system

$$
\frac{d x^{1}}{\xi^{1}(x)}=\frac{d x^{2}}{\xi^{2}(x)}=\ldots=\frac{d x^{m}}{\xi^{m}(x)}
$$

whose general solution $\zeta^{i}(x)=c_{i}, i=1, \ldots, m-1$ provides the functionally independent local invariants. When the action of $G$ is semiregular on the m-dimensional manifold $M$ with $s$-dimensional orbits 
we have integrable systems, characterized by the Lie derivative. The Lie derivative of a geometric object with respect to a vector field represents the infinitesimal change in the object under the flow induced by the vector field or dynamical system. This concept when applied to a specially interesting form of vector fields to be described below, will provide the key to the symmetry condition of non-linear systems leading to their invariants. The concept of symmetries as sets of mutually commuting flows and their existence implies the existence of consequent conservation laws and is the fundamental key to integrability. They can be related to the involution of the set of vector fields (Frobenius' theorem) and the commutativity of differential operators, of which vector fields the simplest example. For our purposes, the compatibility conditions of certain dynamical systems derived from the symmetry conditions will be the key point for determining the integrability of a system of equations, which functionally will be reflected in a set of integrability conditions that we will describe in section 1.8 .

\subsubsection{Lie symmetries of a system of differential equations.}

The first kind of symmetries we will consider are geometric or point symmetries. The geometric approach to systems of differential equations begins with a smooth $m$-dimensional manifold $M$ where solutions are identified as $p$-dimensional (smooth) sub-manifolds $S \subset M$. Local coordinates on $M=X \times U$ consist of a choice of independent variables $x=\left(x_{1}, \ldots, x_{p}\right) \in X=\mathbf{R}^{p}$, and dependent variables $u=\left(u_{1}, \ldots, u_{q}\right) \in U=\mathbf{R}^{q}$, where $p+q=m$, and a sub-manifold $S$ is given as the graph of a function $u=f(x)$.

In contrast to the algebraic case of invariance sketched in the purely algebraic case 1.1.1 we need to manage how the derivatives of the dependent variables change with the continuous infinitesimal transformations used to implement the invariance conditions 1.1.2. For this we need to set the base spaces including derivatives in the system of differential equations. This is achieved by the prolongation of the space of variables, infinitesimal generators and corresponding group actions. The derivatives of the dependent variables are represented by $u_{J}^{\alpha}=\partial_{J} f^{\alpha}(x)$, where $\alpha=1, \ldots, q$ and $J$ is a multi-index of order $0 \leq \# J \leq n$. These form a system of local coordinates, collectively denoted by $\left(x ; u^{(n)}\right)$, on the $n$-th order (extended) jet bundle $J^{n} \rightarrow M$. We will work, following Olver [34], with a greatly simplified version of the theory of jet bundles occurring in the differential-geometric theory of PDEs, by using exclusively Euclidean spaces.

Following Olver [34], the jet bundle is defined as the set of equivalence classes of $p$-dimensional sub-manifolds $S \subset M$. Here $S \sim S^{\prime}$ defines the same equivalence class or jet $j_{n} S \in J^{n}$ at a point $z \in S \cap S^{\prime}$ 
when the two sub-manifolds have $n$-th order contact at $z$. A system of differential equations

$$
\Delta_{v}\left(x ; u^{(n)}\right)=0, \quad v=1, \ldots, s
$$

is regular if the Jacobian matrix of $\Delta_{v}$ has maximal rank $s$ at all $\left(x ; u^{(n)}\right)$ satisfying the system. A regular system can be viewed as a submanifold $S_{\Delta}=\left\{\Delta_{v}\left(x ; u^{(n)}\right)=0\right\} \subset J^{n}$. A classical (smooth) solution is a sub-manifold $S \subset M$ whose jet $j_{n} S \subset S_{\Delta}$. The system is locally solvable if there exists a smooth solution passing through each point $\left(x ; u^{(n)}\right) \in S_{\Delta}$.

Let $G$ be an $r$-dimensional Lie group acting smoothly on $M$. Since $G$ preserves contact between sub-manifolds, there is an induced action, denoted $G^{(n)}$, on $J^{n}$, called the $n$-th order prolonged action, which tells us how $G$ acts on the derivatives of functions. By definition, this action defines a symmetry group of a system of differential equations $S_{\Delta}$ if it maps solutions to solutions, i.e: is a symmetry. Assuming local solvability this occurs if and only if $S$ is a $G^{(n)}$-invariant subset of $J^{n}$. As mentioned before, a connected Lie group action is entirely determined by its infinitesimal generators or prolonged vector fields, which are vector fields on the manifold $M$ and can be identified with the Lie algebra $\mathfrak{g}$ of $G$. Each vector field

$$
\mathbf{v}=\sum_{i=1}^{p} \xi^{i}(x, u) \frac{\partial}{\partial x^{i}}+\sum_{\alpha=1}^{q} \phi_{\alpha}(x, u) \frac{\partial}{\partial u_{J}^{\alpha}} \in \mathfrak{g}
$$

generates a one-parameter subgroup. The infinitesimal generator of the corresponding $n$-th prolonged one-parameter subgroup is a vector field

$$
p r^{(n)} \mathbf{v}=\sum_{i=1}^{p} \xi^{i}(x, u) \frac{\partial}{\partial x^{i}}+\sum_{\alpha=1}^{q} \sum_{j=0}^{\# J} \phi_{\alpha}^{J}\left(x, u^{(j)}\right) \frac{\partial}{\partial u_{J}^{\alpha}}
$$

on $J^{n}$. The explicit formula for the coefficients $\phi_{J}^{\alpha}$ of the prolongation $p r^{(n)} \mathbf{v}$ in terms of the derivatives of the coefficients $\xi^{i}(x, u), \phi_{\alpha}(x, u)$ of $\mathbf{v}$ was given in Olver [37] as

$$
\phi_{\alpha}^{J}\left(x, u^{(n)}\right)=D_{J}\left(\phi^{\alpha}-\sum_{i=1}^{p} \xi^{i} u_{i}^{\alpha}\right)+\sum_{i=1}^{p} \xi^{i} u_{J, i}^{\alpha}
$$

or the recursion formula

$$
\phi_{\alpha}^{J, k}\left(x, u^{(n)}\right)=D_{k} \phi_{\alpha}^{J}-\sum_{i=1}^{p} D_{k} \xi^{i} \cdot u_{J, i}^{\alpha}
$$

with

$$
u_{i}^{\alpha}=\frac{\partial u^{\alpha}}{\partial x^{i}}=\frac{\partial u^{\alpha}}{\partial x^{i}}, \quad u_{J, i}^{\alpha}=\frac{\partial u_{J}^{\alpha}}{\partial x^{i}}=\frac{\partial^{k+1} u^{\alpha}}{\partial x^{i} \partial x^{j_{1}} \cdots \partial x^{j_{k}}}
$$

Note that $t$ is not defined as a different variable to any $x^{i}$. This will have a crucial role as we will see in 1.4 
being

$$
\begin{aligned}
D_{i} & =\frac{\partial}{\partial x^{i}}+\sum_{\alpha=1}^{q} \sum_{J} u_{J, i}^{\alpha} \frac{\partial}{\partial u_{J}^{\alpha}} \\
D_{J} & =D_{j_{1}} D_{j_{2}} \ldots D_{k}
\end{aligned}
$$

the total derivative w.r.t $x^{i}$ differential operator, which differs from partial derivatives by the well known chain rule. For a smooth function of $x, u$ and the derivatives of $u$ up to order $n, P=P\left(x, u^{(n)}\right)$, this operator is defined on an open subset of $M^{(n)} \subset X \times U^{(n)}$ as the unique smooth function $D_{i} P\left(x, u^{(n+1)}\right)$ defined on $M^{(n+1)}$ with the property that if $u=f(x)$ is any smooth function, then

$$
D_{i} P\left(x, p r^{(n+1)} f(x)\right)=\frac{\partial}{\partial x^{i}}\left\{P\left(x, p r^{(n)} f(x)\right)\right\}
$$

this is, by differentiating $P$ w.r.t $x^{i}$ while treating $u$ and its derivatives as independent functions of $x$.

Smooth prolonged vector fields have the additional properties of linearity and compatibility with the Lie bracket

- $p r^{(n)}(\alpha \mathbf{v}+\beta \mathbf{w})=\alpha p r^{(n)} \mathbf{v}+\beta p r^{(n)} \mathbf{w}, \quad \alpha, \beta \in \mathbf{R}$

- $p r^{(n)}[\mathbf{v}, \mathbf{w}]=\left[p r^{(n)} \mathbf{v}, p r^{(n)} \mathbf{w}\right]$

thus forming a Lie algebra and determining a Lie group of prolonged transformations. The study of these groups having important applications to the resolution of differential equations, to mention one, by the reduction or order. It is also interesting the study of the correspondent Lie algebras and their solvability and the relation with the solvability of the equation possessing such a symmetry group, being study fields out of the scope of this thesis. The interested reader can read more on these topics in Morales-Ruiz [31] and Olver [34, 35].

The generic infinitesimal symmetry criterion for prolonged vector

This is the first version of the previously introduced generic symmetry condition, which will evolve to more elaborated formulae for the different types of symmetries under consideration. fields can be formulated as follows [34]

Theorem 1. A connected group of transformations $G$ is a symmetry group of the regular system of differential equations $\Delta$ if and only if

$$
\operatorname{pr}^{(n)} \mathbf{v}\left[\Delta_{v}\left(x ; u^{(n)}\right)\right]=0 \quad v=1, \ldots, s
$$

whenever $\Delta_{v}\left(x ; u^{(n)}\right)=0$ for every $\mathbf{v} \in \mathfrak{g}$.

This theorem allows the computation of the coefficients of the prolonged infinitesimal generator $p r^{(n)} \mathbf{v}$ of an hypothetical connected symmetry group of transformations of the system of differential equations $\Delta$, which are unknown functions of $x$ and $u$, and its partial 
derivatives. The solution of these determining equations will determine the most general infinitesimal symmetry of the system and are used to find the form of geometrical (point) symmetries.

The prolongation formula 1.1.5, coupled with the infinitesimal symmetry criterion in theorem 1 and the total derivative operator, allows us to explicitly compute the symmetry groups of almost any system of differential equations.

This initial version of the symmetry criterion for systems of differential equations shows how Lie symmetry theory is extended from algebraic to differential systems and provides systematic and effective mechanism for computing a wide variety of new solutions from known solutions. Further applications are to finding explicit groupinvariant solutions, to determining conservation laws, and specially to the classification of differential equations with given symmetry groups. In this work we focus of the symmetry methods to determine the integrability of generic differential equations. For this, we will describe further generalizations of the symmetry concept in this chapter and how they are applied to integrability in the next one.

\subsubsection{Characteristics and Evolutionary form of symmetries}

An more compact and computationally most effective way of writing formula 1.1.6 for the prolongation coefficients is achieved by introducing the concept of characteristic of a symmetry. Defining, from 1.1.4

$$
Q_{\alpha}\left(x, u^{(1)}\right)=\phi_{\alpha}(x, u)-\sum_{i=1}^{p} \xi^{i}(x, u) u_{i}^{\alpha}, \quad \alpha=1, \ldots, q
$$

as the characteristic of the vector field $\mathbf{v}$, the prolongation coefficients take the form

$$
\phi_{\alpha}^{J}\left(x, u^{(n)}\right)=D_{J} Q_{\alpha}+\sum_{i=1}^{p} \xi^{i} u_{J, i}^{\alpha}
$$

and the prolongation formula changes to

$$
p r^{(n)} \mathbf{v}=p r^{(n)} \mathbf{v}_{Q}+\sum_{i=1}^{p} \xi^{i} D_{i}
$$

with

$$
\mathbf{v}_{Q}=\sum_{\alpha=1}^{q} Q_{\alpha}\left(x, u^{(1)}\right) \frac{\partial}{\partial u^{\alpha}}
$$

and

$$
p r^{(n)} \mathbf{v}_{Q}=\sum_{\alpha=1}^{p} \sum_{J} D_{J} Q_{\alpha} \frac{\partial}{\partial u_{J}^{\alpha}}
$$

These will represent a crucial simplification of the prolongation formula and therefore in the computation of symmetry groups, specially 
for evolution equation, when coupled with the concept of evolutionary form of symmetries, forward in this introduction.

This is due to the property of any generalized vector field $\mathbf{v}$ being a symmetry of a system if and only if its evolutionary representative $\mathbf{v}_{Q}$ is, which is easily checked by using 1.1.14, as $D_{i} \Delta_{v}$ vanishes as a differential consequence $\Delta^{(k)}=0$ of the system $\Delta$. We will make use of this again forward for further simplification of prolongations when obtaining symmetry conditions.

\subsection{GENERALIZED SYMMETRIES AND RECURSION OPERATORS}

Generalized or contact symmetries were first considered by S. Lie at the end of 19th century and E. Noether in the beginning of the 2oth century as an application to variation problems (Noether [32]). Also called high order or higher symmetries allow dependence of the coefficients $\xi^{i}, \phi_{\alpha}$ of the infinitesimal generator $\mathbf{v}$ on the derivatives $u_{J}^{\alpha}$

$$
\mathbf{v}=\sum_{i=1}^{p} \xi^{i}\left(x, u, u_{J}^{\alpha}\right) \frac{\partial}{\partial x^{i}}+\sum_{\alpha=1}^{q} \phi_{\alpha}\left(x, u, u_{J}^{\alpha}\right) \frac{\partial}{\partial u_{J}^{\alpha}}
$$

defining a generalized vector field which corresponds to generalized local group of transformations whose infinitesimal generators can be proved, for maximal rank non-degenerate systems, to form a Lie algebra as in the geometric case of section 1.2.

The symmetry criterion of invariance has the same expression 1.I.II as in the case of Lie-geometrical symmetries, and the method for calculation of the symmetry group of a system of differential equations described for geometric symmetries above is also valid for generalized symmetries which are defined as the generalized vector fields 1.2.1 satisfying the symmetry condition 1.1.11.

Generalized symmetries play an essential role in the study of integrable equations, inverse scattering and soliton solutions and in the general framework of linearization and integration. The key observation about these symmetries is that if an equation possesses a generalized symmetry, then it usually possesses an infinite sequence of generalized symmetries, as was conjectured by A. Fokas in [12]. Counterexamples to this conjecture have been found (cf. Kamp and Sanders [20]) The existence of such an infinite sequence or hierarchies has many consequences on the integrability properties of the system.

These infinite sequences are generated through recursion operators, which are linear operators that produce a new symmetry from a given one recursively. 
For generalized symmetries of a normal analytic system the symmetry condition 1.1.11 can be expressed as the existence of linear differential operators $\mathscr{D}_{\nu \mu}=\sum_{i, J} P[u]_{v \mu}^{J} D_{J}^{i}$ such that

$$
\operatorname{pr} \mathbf{v}\left(\Delta_{\nu}\right)=\sum_{\mu=1}^{l} \mathscr{D}_{\nu \mu} \Delta_{\mu}
$$

holds for all functions $u=f(x)$.

Coefficients $P[u]$ denoting expressions depending on $x, u$ and derivatives $u_{J}$ of $u$ as in $P\left(x, u^{(m)}\right)$ up to a finite but unspecified order $m$, are called differential functions in analogy with the differential polynomials of differential algebra.

The set of differential functions forms an algebra $\mathscr{A}$ defined as the space of smooth functions $P[u]$, with the fundamental operators $\frac{\partial}{\partial x^{i}}$, $\frac{\partial}{\partial u_{J}^{\alpha}}, D_{i}$ and the Divergence, Euler operator, etc. acting linearly on it. Multidimensional algebras of differential functions are defined as $\mathscr{A}^{k}$, the space of $k$-tuples $\mathbf{P}[u]=\left(P_{1}[u], \ldots, P_{k}[u]\right)$. Generalized vector fields have their prolongation inside the algebra of differential functions (with finite order), with the symmetry condition for generalized symmetries $\operatorname{pr} \mathbf{v}[\boldsymbol{\Delta}]=0$ depending on $(n+m)$-th order derivatives.

\subsection{EVOLUTIONARY VECTOR FIELDS AND SYMMETRIES}

An important simplification to the calculus of generalized symmetries is introduced by evolutionary vector fields, in which the coefficients $\xi^{i}[u]$ of the $\frac{\partial}{\partial x^{i}}$ are zero. This considerably simplifies the computation of prolongations. Their characteristic, infinitesimal generator coefficients and prolongation formula, are as defined in 1.1.12, while the coefficients in 1.1.15 are differential functions:

$$
\mathbf{v}_{Q}=\sum_{\alpha=1}^{q} Q_{\alpha}[u] \frac{\partial}{\partial u^{\alpha}}
$$

they are called evolutionary fields and $Q$ is its characteristic. Having $\xi^{i}=0$ in $\mathbf{v}_{Q}$, as per 1.1.12 we have prolongation coefficients as

$$
\phi_{\alpha}^{J}=D_{J} \phi_{\alpha}
$$

and

$$
\operatorname{pr} \mathbf{v}_{Q}=\sum_{\alpha, J} D_{J} \phi_{\alpha} \partial_{u_{J}^{\alpha}}
$$

thus determining the same symmetry than $\mathbf{v}$ (cf. I.I.II and 1.1.14). The evolutionary representative $\mathbf{v}_{Q}$ of a generalized vector field $\mathbf{v}$ simplifies the prolongation formula 1.1.5 to

$$
\operatorname{pr} \mathbf{v}\left[\Delta_{v}\right]=\operatorname{pr} \mathbf{v}_{Q}\left[\Delta_{v}\right]
$$


and therefore both the symmetry condition and the symmetry group computation algorithm, for which order $m$ of characteristics $Q\left(x, u^{(m)}\right)$ needs to be fixed a priori. The higher $m$, the more possible generalized symmetries are to be found.

Evolutionary vector fields also have the interesting property of commuting with the total derivative; for any differential function $P[u]$

$$
\sum_{\alpha, J} D_{J} Q_{\alpha}[u] \frac{\partial P}{\partial u_{J}^{\alpha}}=\operatorname{pr} \mathbf{v}_{Q}(P)
$$

determines the infinitesimal change in $P$ under the one-parameter group generated by $\mathbf{v}_{Q}$ holding the following property

$$
\operatorname{pr} \mathbf{v}_{Q}\left[D_{i} P\right]=D_{i}\left(\operatorname{pr} \mathbf{v}_{Q}(P)\right)
$$

which in turn proves the fact that if $\mathbf{v}_{Q}$ is a symmetry of the system $\Delta$, then it is also a symmetry of any prolongation $\Delta^{(k)}$. For all the equations in $\Delta^{(k)}$ we have $D_{J} \Delta_{v}=0$, and by 1.3 .3

$$
\operatorname{pr} \mathbf{v}_{Q}\left(D_{J} \Delta_{v}\right)=D_{J}\left(\operatorname{pr} \mathbf{v}_{Q}\left(\Delta_{v}\right)\right)=0
$$

whenever $u$ is a solution since $p r \mathbf{v}_{Q}\left(\Delta_{v}\right)$ vanishes on solutions.

We can at this point sketch the procedure of computation of generalized symmetries as:

1. Put the symmetry in evolutionary form;

2. Fix the order $m$ of the characteristic $Q$;

3. Write the symmetry condition for the system $\Delta$, as

$$
\begin{aligned}
& \operatorname{pr} \mathbf{v}_{Q}\left[\Delta_{v}\right]= \\
& \quad=\left(Q \partial_{u}+Q_{x} \partial_{u_{x}}+Q_{t} \partial_{u_{t}}+Q_{x t} \partial_{u_{x t}}+Q_{x x} \partial_{u_{x x}}+\ldots\right)\left[\Delta_{v}\right]=0
\end{aligned}
$$

4. Deal with the occurrence of trivial symmetries, by eliminating any superfluous derivatives in $Q$ by substitution using the prolongations $\Delta^{(k)}$ (or differential consequences) of the system,

5. Extracting conditions on coefficients of $u_{J}$ we have the equations determining the symmetries.

The whole theory of infinitesimal invariance, prolongation and action of vector fields and symmetry groups is extended to generalized symmetries, being the main result that the set of generalized symmetries of a system of differential equations with the Lie bracket for generalized vector fields is an algebra with the Lie bracket defined for all differential functions $P \in \mathscr{A}$ as

$$
\operatorname{pr}[\mathbf{v}, \mathbf{w}](P)=\operatorname{pr} \mathbf{v}[p r \mathbf{w}(P)]-\operatorname{pr} \mathbf{w}[p r \mathbf{v}(P)]
$$


This fact is proved in detail in Olver [34] and provides a systematic means of determining all the generalized symmetries of a given order of a system of differential equations but suffers the drawback that the order of the derivatives on which the coefficients of the symmetry depend must be specified in advance. Therefore, the method cannot simultaneously generate all generalized symmetries of the system. In turn, the method of generating symmetries based on the notion of a recursion operator introduced in this section provides infinite hierarchies of symmetries although it is not fully constructive, being the only available approach a combination of the first method on lower orders and a certain amount of inspired work for the deduction of the form of a recursion operator based in the lower order symmetries. However a concrete criterium for recursion operators will be given after introducing formal calculus and the formal symmetry approach to integrability in the next chapters.

In the next section we present some examples of the two approaches for the case of evolution equations, which historically have inspired the development of the symmetry approach framework.

\subsection{SYMMETRIES OF EVOLUTION EQUATIONS}

A system $\Delta_{v}\left(x, u^{(n)}\right)$ of $q$ evolution equations

$$
\frac{\partial u^{v}}{\partial t}=K_{v}[u]=K_{v}\left(x, u^{(n)}\right), \quad v=1, \ldots, q
$$

with r.h.s $\mathbf{K}[u] \in \mathscr{A}^{q}$ depending on $x \in \mathbb{R}^{p}, u \in \mathbb{R}^{q}$ and $x$-derivatives of $u$ only, has $m$-th order symmetries that can be written as $\mathbf{Q}[u]=$ $\mathbf{Q}\left(x, t, u^{(m)}\right)$ depending on $x, t$, and $x$-derivatives of $u$ only, after substituting differential consequences $\Delta^{(k)}=0$.

The generalized symmetry condition for this type of systems has the following characteristics and properties:

- 1.2.2 for 1.4.1 can be expressed as

$$
D_{t} Q_{v}=p r \mathbf{v}_{\mathbf{Q}}\left(K_{v}\right), \quad v=1, \ldots, q
$$

and will in general depend on $(n+m)$-order derivatives.

- $\mathbf{v}_{K}$ itself is always a symmetry of $u_{t}=K[u]$. It reflects the time translation symmetry group generated by $\partial_{t}$ of autonomous dynamical systems.

- An evolutionary vector field $\mathbf{v}_{Q}$ is a symmetry of the system if and only if

$$
\frac{\partial \mathbf{v}_{Q}}{\partial t}+\left[\mathbf{v}_{K}, \mathbf{v}_{Q}\right]=0
$$


When $\mathbf{Q}\left(x, u^{(m)}\right)$ does not depend on $t$, then 1.4.2 reduces to the condition that the two vector fields $\mathbf{v}_{K}$ and $\mathbf{v}_{Q}$ commute:

$$
\left[\mathbf{v}_{K}, \mathbf{v}_{Q}\right]=0
$$

which is equivalent to have the correspondent one-parameter symmetry groups commuting.

This defines evolutionary symmetries and represents the foundation of the symmetry approach to systems in which the differential consequences $\Delta^{(k)}$ allow the $t$-independence of the r.h.s $\mathbf{K}[u]$ of $\Delta$, as we will see in chapter 2 .

\subsection{INTEGRABILITY TYPES AND DEFINITIONS}

There is not a definitive definition of integrability and this matter is the central point of past and present research. Full books are devoted to the subject, among which the closest publication to our interests is Calogero [8]. In this section we quote several generic definitions of integrability and explain which of them we will apply in our calculations. Our concept of integrability is invariant under change of variables by contact transformations (chapter 3 ) and it will be the key to classification of integrable equations and by extension of the techniques derived from this approach, to the classification of integrable Lagrangian systems.

Integrability in our context means that there is a method to obtain explicit solutions to the differential equations involved. When this method is based in changes of variables, we talk, following Calogero, about $C$-integrability, while methods based in spectral transformations (like the inverse scattering method) regard to S-integrability, Calogero [8].

The existence and constructability of a method goes beyond the existence and uniqueness theorems for analytic solutions and requires additionally the solutions to be constructable. In this sense Galois theory and Picard-Vessiot theory (see Morales-Ruiz [31]) provide an abstract setting satisfactory from algebraic point of view, presently applicable only to ordinary differential equations. Another, more geometrical theory of integrability, applicable to systems of vector fields and their dual objects, differential forms (i.e. certain first order partial differential equations), there is a relationship between involution as existence of integral sub-manifolds satisfying Frobenius theorem and integrability in the theory of differential systems generated by differential forms which constitute the deepest theoretical justification of the integrability conditions.

However, in this thesis we will not delve into the construction of symmetry algebras, the jet space geometry, differential forms or spectral transforms theories mentioned, but rather research into the existence of generalized symmetries and canonical conservation laws in 
concrete cases as necessary conditions to integrability. These properties are common to integrable and linear systems.

Any linear partial differential equation admits an infinite-dimensional symmetry group formed by addition of solutions and conversely a system of partial differential equations can be linearized if and only if it has an infinite-dimensional symmetry group of the proper form. However, some non-linear differential equations are integrable and the integrability problem usually is preceded by linearization. The Poincaré variational equation of the flow of a finitedimensional dynamical system (i.e. systems of ODEs) leads to a general principle (cf. Morales-Ruiz [31]) for accepting the conjecture of the linearization being integrable if the system is, which translates into flow commutativity conditions or vanishing of Lie derivatives, introduced in preceding sections, leading to symmetry conditions which are constructed from the geometric conditions of invariance certain objects under the Lie derivative.

The following definition of integrability, applicable to any partial differential equation, can be found in Olver [34], Mikhailov, Shabat, and Sokolov [26] and Heredero [15] and although it is not the final definition we will use in this project, it is the appropriate definition for generalized symmetries, closely related to recursion operators as we have seen so far in this introduction.

An evolution equation is considered integrable

if it admits infinitely many symmetries.

Like linear equations, integrable equations admit infinite symmetries. As we tried to show in this introduction, vector fields generating infinitesimal symmetries commute and generate a Lie algebra guaranteeing the existence of solution sub varieties when it is possible to generate new vector fields from the ones in the equation. At the algebraic level this is the function of recursion operators we introduced in section 1.2, keeping in mind that finding recursion operators for non-linear equations is far away from being algorithmically computable.

The formal method for finding infinitely many symmetries of a given equation is not, however, to find recursion operators by inspection of the equations symmetries. It is described in Mikhailov, Shabat, and Sokolov [26] and summarized in the following. For this we will follow the approach presented in Heredero [15] and Heredero, Sokolov, and Svinolupov [17]. The symmetry-based definition of integrability will be given after introducing formal calculus, pseudodifferential operators and formal symmetries. This method has been used to obtain classification theorems for second (Svinolupov [47]) and third (Heredero [15]) order evolution equations and provides the fundamentals for the extension of this techniques to non evolutionary equations and integrable Lagrangian systems as detailed in Heredero, Shabat, and Sokolov [16].

Algorithms for computability of recursion operators can be derived from the algebraic properties of the space of solutions 


\subsection{FORMAL SYMMETRIES AND INTEGRABILITY OF EVOLUTION EQUATIONS}

Using the method for determining the generalized symmetries of a system of differential equations presented in 1.2 allows the determination of the symmetry group of the system, up to the maximum order $m$ of derivatives specified in the coefficients $\xi^{i}\left(x, u, u_{J}^{\alpha}\right), \varphi^{\alpha}\left(x, u, u_{J}^{\alpha}\right)$ of the symmetry generator $\mathbf{v}$ (1.2.1). This method is on one hand fully constructive but on the other hand has the drawback of specifying the order $m$ of symmetries in advance. When combined with the concept of recursion operator the resulting new methodology allows us to find infinite hierarchies of generalized symmetries provided that such a recursion operator (which is easily verified but not systematically constructed) is given. In this way, the calculus methods for symmetries make use of differential algebra (see Ritt [40] for a comprehensive introduction to the subject).

Let the system under consideration be expressed with a linear differential operator $\Delta[u]=0$. A second operator $\mathscr{R}$ is a recursion operator for the system if and only if $Q=\mathscr{R}[u]$ is the characteristic of a generalized symmetry. At this point the linearization of the system will result in the general technique of formal symmetries:

- for linear systems, the existence of the trivial scaling symmetry group $(x, u) \mapsto(x, \lambda u)$ guarantees the existence of the symmetry with characteristic $Q_{0}=u$, with correspondent generator $\mathbf{v}=u \partial_{u}$, and trivially makes $Q=\mathscr{R}[u]$ a symmetry, and conversely if $\mathbf{v}_{Q}$ is a symmetry then by 1.1.11 and the linearity of $\Delta$

$$
\operatorname{pr} \mathbf{v}_{Q}(\Delta[u])=\Delta[Q]=\Delta \mathscr{R}[u]
$$

- for non-linear systems, the Fréchet derivative differential operator

$$
\left(\mathrm{D}_{K}\right)_{\mu \nu}=\sum_{J} \frac{\partial K_{\mu}}{\partial u_{J}^{v}} D_{J}
$$

like the prolongation formula found for generalized symmetries, determines the infinitesimal variation in $K$ under the action of the one-parameter group generated by $\mathbf{v}_{Q}$ :

$$
\mathrm{D}_{K}(Q)=\operatorname{pr} \mathbf{v}_{Q}(K)
$$

This expression provides an alternative form of the symmetry condition 1.1.11:

$$
\mathrm{D}_{K}(\Delta)=0
$$

on solutions. Additional properties of the Fréchet operator follow from $D$ being a derivation in the algebra $\mathscr{A}$ of differential functions.

For any $P[u], Q[u] \in \mathscr{A}$, we have 
- Leibniz rule: $\mathrm{D}_{P Q}=P \mathrm{D}_{Q}+Q_{P}$

- Linearization: $\mathrm{D}_{\mathscr{D} P}=\mathscr{D} \cdot \mathrm{D}_{P}$ for any constant coefficient differential operator $\mathscr{D}$.

- Identity for linear differential functions

$$
P[u] \text { linear } \Leftrightarrow \mathrm{D}_{P}=P
$$

Differential operators have the form of "polynomials" with differential functions as coefficients as defined in 1.2. The following two of their interesting properties are commonly used in the formal symmetry approach:

- The time derivative of a generic matrix differential operator $\mathscr{D}_{\nu \mu}=\sum_{J} P[u]_{v \mu}^{J} D_{J}$ is computed as

$$
\mathscr{D}_{t}=\sum_{J} D_{t}\left(P[u]_{v \mu}^{J}\right) D_{J}
$$

For evolution equations will provide the key criterion for finding generic recursion operators as the vanishing of their Lie derivative with respect to the equation's vector field.

- The Lie derivative of a generic (matrix) differential operator $\mathbf{R}$ with respect to a generalized vector field $\mathbf{v}_{\mathbf{Q}}$ is the differential operator

$$
\mathbf{v}_{Q}[[\mathbf{R}]]=\mathbf{R}_{t}+\left[\mathbf{R}, \mathrm{D}_{Q}\right]=\operatorname{pr} \mathbf{v}_{Q}(\mathbf{R})+\left[\mathbf{R}, \mathrm{D}_{Q}\right]
$$

With these tools in hand we are now able to characterize a recursion operator $\mathscr{R}$ for a system of differential equations $\Delta$ as a linear operator such that

$$
\mathrm{D}_{\Delta} \cdot \mathscr{R}=\widetilde{\mathscr{R}} \cdot \mathrm{D}_{\Delta}
$$

for all solutions $u$ to $\Delta$, where $\widetilde{\mathscr{R}}$ is a linear differential operator.

In the case of evolution equations $u_{t}=K[u]=K\left(x, u^{(n)}\right)$ we have $\Delta[u]=u_{t}-K[u]$ and $\mathscr{R}=\widetilde{\mathscr{R}}$. Linearization trough the Fréchet derivative gives

$$
\mathrm{D}_{\Delta}=D_{t}-\mathrm{D}_{K}
$$

and 1.6 .6 reduces the condition for a recursion operator to

$$
\mathscr{R}_{t} \doteq\left[\mathrm{D}_{K}, \mathscr{R}\right]
$$

which is the same as requiring the vanishing of the Lie derivative of

This condition represents the cornerstone of the symmetry approach to integrability the recursion operator under the flow generated by the evolutionary vector field $\mathbf{v}_{K}$, this is having $\mathscr{R}$ unchanged by the flow determined by the equation,

$$
\mathbf{v}_{Q}[[\mathscr{R}]]=0
$$


Thus, through the use of the Fréchet operator we have generalized the way of finding infinitely many symmetries to non-linear equations.

At this point we take the following definition of integrability

An evolution equation is considered integrable if it admits a recursion operator.

\subsection{FORMAL SYMMETRIES AND FORMAL RECURSION OPERATORS}

For some nonlinear equations, such as $\mathrm{KdV}$ equation introduced above, the the class of recursion operators needs to be enlarged to include formal pseudo-differential operators, which admit negative powers of $D_{x}$. This allowance extends our definition of a differential operator given in 1.2 to a wider ring of differential operators that can be defined as infinite formal series

$$
\mathscr{L}=L_{n} D_{x}^{n}+L_{n-1} D_{x}^{n-1}+\ldots+L_{0}+L_{-1} D_{x}^{-1}+\cdots
$$

whose coefficients are differential functions $L_{i}=L_{i}[u]$, and the integral operator $D_{x}^{-1}$ is the formal inverse of the total derivative $D_{x}$, with the following properties.

- Its action is only defined on those differential functions which are total derivatives (so if $Q=D_{x} R$, then we set $R=D_{x}^{-1} Q$ )

- Identity in $\mathscr{A}$ is an order 0 differential operator or operators

$$
P[u] D_{x} \cdot D_{x}^{-1}=P[u] D_{x}^{0}=P[u] D_{x}^{-1} \cdot D_{x}=P[u]
$$

And the usual rules in the algebra of differential operators:

- The product $D_{x}^{i} \cdot D_{x}^{j}=D_{x}^{i+j}$ is linear, non-commutative, with

- Leibniz rule

$$
\begin{aligned}
& D_{x} Q=Q D_{x}+Q^{\prime} \\
& D_{x}^{2} Q=Q D_{x}^{2}+2 Q^{\prime} D_{x}+Q^{\prime \prime} \\
& \text { with } Q^{\prime}=D_{x}(Q), \quad Q^{\prime \prime}=D_{x}^{2}(Q)
\end{aligned}
$$

$$
\begin{aligned}
D_{x}^{n} Q & =\sum_{k=0}^{n}\left(\begin{array}{l}
n \\
k
\end{array}\right) D_{x}^{(k)}(Q) D_{i}^{n-k} \\
= & Q D_{x}^{(n)}+n D Q D_{x}^{(n-1)}+\ldots+n D^{(n-1)} Q D_{x}+D_{x}^{(n)} Q \\
\text { with } Q^{(k)}= & D_{x}^{k}(Q)
\end{aligned}
$$

- associativity

$$
D_{x}^{n} D_{x}^{m} Q=D_{x}^{n+m} Q=\sum_{k=0}^{n+m}\left(\begin{array}{c}
n+m \\
k
\end{array}\right) Q^{(k)} Q D_{i}^{n+m-k}
$$


The allowance of operating with $D_{x}^{-1}$ expands the ring of differential operators (defined as finite sums $\mathscr{D}=\sum_{i=0}^{n} P_{i}[u] D_{x}^{i}$ where the coefficients $P_{i}[u]$ are differential functions) to that of pseudo-differential operators, infinite sums, analogous to Laurent series, and with above properties valid for the product of pseudo-differential operators and the following additional properties

- $\operatorname{order}\left(\mathscr{L}=\sum_{i=-\infty}^{n} P_{i}[u] D_{x}^{i}\right)=n$, being order 0 operators differential functions, also called multiplication operators.

- $D_{x}^{-1}$ generates itself more terms on decreasing powers of $D_{x}^{-1}$ making for $P[u] \in \mathscr{A}, \mathscr{L}(P[u]) \notin \mathscr{A}$ as for

$$
\begin{aligned}
D_{x}^{-1} Q & =Q D_{x}^{-1}-D_{x}^{-1} Q^{\prime} D_{x}^{-1} \\
& =Q D_{x}^{-1}-Q^{\prime} D_{x}^{-2}+D_{x}^{-1} Q^{\prime \prime} D_{x}^{-2} \\
& =\ldots \\
& =\sum_{i=0}^{\infty}(-1)^{i} Q^{(i)} D_{x}^{-i-1}
\end{aligned}
$$

which explains the reason of allowing infinite series in the definition of pseudo-differential operators. As the basic example for $Q[u]=u$ we have

$$
\begin{aligned}
D_{x}^{-1} u & =u D_{x}^{-1}-u_{x} D_{x}^{-2}+u_{x x} D_{x}^{-3}-\ldots \\
& =\sum_{i=0}^{\infty}(-1)^{i} u_{i} D_{x}^{-i-1}
\end{aligned}
$$

- Commutator

$$
[\mathscr{D}, \mathscr{E}]=\mathscr{D} \cdot \mathscr{E}-\mathscr{E} \cdot \mathscr{D}
$$

with $\operatorname{order}[\mathscr{D}, \mathscr{E}] \leq \operatorname{order}(\mathscr{D})+\operatorname{order}(\mathscr{E})-1$

- Existence of inverse $\mathscr{D}^{-1}=\mathscr{E}$, with $\operatorname{order}(\mathscr{E})=-\operatorname{order}(\mathscr{D})$, for every pseudo-differential operator $\mathscr{D} \neq 0$

$$
\mathscr{E}=\frac{1}{P_{n}} D_{x}^{-n}+Q_{1} D_{x}^{-n-1}+Q_{2} D_{x}^{-n-2}+\ldots
$$

where $P_{n}$ is the highest order coefficient of $\mathscr{D}$, and $Q_{i}$ follow after the integration of a system of equations derived from $\mathscr{D}$. $\mathscr{E}=1$, with the 0 pseudo-differential operator having order $-\infty$. Being the product non-commutative, $\mathscr{E}$ is a right inverse and also a left inverse.

- Existence of $\mathrm{n}$-th root $\mathscr{E}=\sqrt[n]{\mathscr{D}}$ for every pseudo-differential operator of order $n>0$

$$
\mathscr{E}=\sqrt[n]{P_{n}} D_{x}+Q_{0}+Q_{1} D_{x}^{-n-1}+Q_{2} D_{x}^{-n-2}+\ldots
$$

These two properties have remarkable consequences and provide the expanded ring of operators with additional structure of $a$ non-commutative field. 
where $Q_{i}$ follow after the integration of a system of equations derived from $\mathscr{E}^{n}=\mathscr{D}$. This property allows the definition of fractional powers $(\sqrt[n]{\mathscr{D}})^{i}=\mathscr{D}^{\frac{i}{n}}$ for all integers $i$ and any $n$-th order pseudo-differential operator.

For evolution equations $u_{t}=K[u]=K\left(x, u^{(n)}\right)$ the symmetry condition obtained from 1.4.3 implies that a $t$-independent evolutionary vector field $\mathbf{v}_{Q}$ determines a (generalized, $m$-th order) symmetry if and only if the flows commute, which has the infinitesimal formulation

$$
\operatorname{pr} \mathbf{v}_{K}(Q)-\operatorname{pr} \mathbf{v}_{Q}(K)=0
$$

A simple but very important observation is that whenever this condition holds, its linearization by the Fréchet derivative will hold as well, leading us to obtain the symmetry condition in formal series allowing the order $m$ of the initial generalized symmetry to grow, which overcomes the drawback of fixing the order $m$ for a generalized symmetry in the procedure in section 1.3.

Applying the linearization technique described above, by taking the Fréchet derivative of 1.7.2, we obtain the differential operators version of the symmetry condition as

$$
\operatorname{pr} \mathbf{v}_{K}\left(\mathrm{D}_{Q}\right)-\operatorname{pr} \mathbf{v}_{Q}\left(\mathrm{D}_{\mathrm{K}}\right)-\left[\mathrm{D}_{\mathrm{K}}, \mathrm{D}_{\mathrm{Q}}\right]=0
$$

For equations having generalized symmetries of order $m \gg n$ the highest order terms in this differential operators equation are the first and third, and replacing the Fréchet derivative of the generalized symmetry $Q$ by an arbitrary pseudo-differential operator $\mathscr{D}$, we obtain the formal series symmetry condition

$$
\mathscr{D}_{t}+\left[\mathscr{D}, \mathrm{D}_{K}\right]=0
$$

in which $\mathscr{D}$ is called a formal symmetry of rank $k$ whenever

$$
\begin{aligned}
\operatorname{order}\left(\mathscr{D}_{t}+\left[\mathscr{D}, \mathrm{D}_{K}\right]\right) \leq \operatorname{order}(K[u])+\operatorname{order}(\mathscr{D}) & -\operatorname{rank}(\mathscr{D}) \\
& =n+m-k
\end{aligned}
$$

This definition combined with 1.7 .3 shows the important relationship between generalized and formal symmetries after the linearization by the Fréchet derivative. For any $m$-th order generalized symmetry with characteristic $Q\left(x, u^{(m)}\right)$ its Fréchet derivative $D_{Q}$ is a rank- $m$ formal symmetry of $u_{t}=K[u]$.

Condition 1.7.4 is again a consequence of the infinitesimal invariance condition, this time being the Lie derivative of the pseudo-differential operator $\mathscr{D}$ vanishing. The higher the rank of the formal symmetry, the more restrictive the conditions imposed to the evolution equation in such a way that a rank $\infty$ formal symmetry is defined as a formal recursion operator for the evolution equation $u_{t}=K$. 
Thus, from the definition of integrability given in 1.5 , we have reached a formal calculus version of it

An evolution equation is called integrable

if it admits a formal recursion operator.

In the framework of the symmetry approach to integrability such a formal symmetry $\mathscr{D}$ with all the conditions above is called a Formal Recursion Operator (FRO). With this as the most powerful tool for generating infinitely many symmetries, the final integrability definition can be expressed now as

An evolution equation is called integrable

if it possesses a non-constant FRO or formal symmetry of rank $\infty$.

\subsection{INTEGRABILITY CONDITIONS}

The integrability conditions for a given scalar evolution equation (or class of equations) are relations derived from the algebraic properties of the space of solutions of 1.7.4, which we can write

$$
\mathscr{D}_{t} \doteq\left[\mathrm{D}_{K}, \mathscr{D}\right]
$$

This equation is the cornerstone of this thesis.

highlighting its applicability on solutions of $u_{t}=K\left(x, u, u_{x}, u_{x x}, \ldots\right)$.

The consequences of these algebraic properties, derived from those of existence of inverse and roots of any pseudo-differential operator which were introduced in section 1.7 make the formal symmetry approach more interesting (from the calculus perspective) than the geometrical framework being described in this chapter.

The reference approach for our calculations will be the standard method of deriving necessary integrability conditions based on the residues of fractional powers of formal recursion operators. We are summarizing these consequences in the following definitions and properties whose proofs can be found in [1].

We will see how the formal calculus techniques derived from the above properties are useful for the expression of integrability conditions of a generic evolution equation when expanding the formal series symmetry condition.

Theorem 2. The set of the formal symmetries of a given equation is a ring in which product is associative and linear. Furthermore,

- if $\mathscr{L}, \mathscr{M}$ are formal symmetries of rank $k$, then

- $\mathscr{L} \mathscr{M}$ is a formal symmetry of rank $k$,

- $\mathscr{L}^{j}$ and $\mathscr{L}^{1 / l}$ are formal symmetries of rank $k$.

- if $Q$ is a generalized symmetry of order $m$, then $D_{Q}$ is a formal symmetry of rank $m$. 
The following lemma, is a consequence of the conservation of rank by operations of linearization, sum, product, inverse and power of pseudo-differential operators and permits the normalization of FROs in the fundamental theorems of the formal symmetry approach, including its extensions as we will see in the following chapters.

Lemma 3. Every formal symmetry can be normalized to a formal symmetry of differential order 1, without changing its rank.

Definition 4. The residue of a $n$-th order FRO

$$
\mathscr{L}=L_{n} D_{x}^{n}+L_{n-1} D_{x}^{n-1}+\ldots+L_{0}+L_{-1} D_{x}^{-1}+\cdots
$$

is defined as the coefficient of $D_{x}^{-1}$, this is the differential function $L_{-1}$.

Theorem 5. Adler Formula, [1]. The residue of the commutator of two pseudo-differential operators is a total derivative,

$$
\operatorname{Res}([\mathscr{L}, \mathscr{M}]) \in \operatorname{Im} D_{x}
$$

or equivalently

$$
\operatorname{Res}([\mathscr{L}, \mathscr{M}])=D_{x} \sigma(\mathscr{L}, \mathscr{M})
$$

This formula implies $\operatorname{Res}([\mathscr{L}, \mathscr{M}])=D_{x} P$ for some differential function $P[u]$ and has important consequences in the interpretation of the algebraic properties of the space of solutions of the symmetry condition 1.7 .4 in the form of conservation laws. We will see how the formal calculus techniques derived from the above properties are useful for the expression of integrability conditions of a generic evolution equation when expanding the formal series symmetry condition in section 1.11.

\subsection{CONSERVATION LAWS AND CONSERVED DENSITIES}

In the same way as a system of differential equations, $\Delta[u]$, a generic variational problem with functional

$$
\mathbb{L}[u]=\int_{\Omega} \mathcal{L}\left(x, u^{(n)}\right) d x
$$

and with $n$-th order Lagrangian density $\mathcal{L}$, admitting a symmetry group $G$ leads to a $G$-invariant system of Euler-Lagrange equations determining the extremals of the variational problem when the Euler operator vanishes

$$
\mathrm{E}(\mathcal{L})=\frac{\partial \mathcal{L}}{\partial u}-D_{x} \frac{\partial \mathcal{L}}{\partial u_{x}}+D_{x}^{2} \frac{\partial \mathcal{L}}{\partial u_{x x}}-\ldots+(-1)^{n} D_{x}^{n} \frac{\partial \mathcal{L}}{\partial u_{n}}(1.9 .1)
$$

Divergence expressions $\operatorname{Div} P=Q \cdot \Delta=0$ vanishing for all solutions $u=f(x)$ of a given system $\Delta$ are called conservation laws. For ordinary systems they take the form $D_{x} P=0$ and determine the first 
integrals or constants of motion. The analogue concept for partial differential equation systems (including variational problems) are classically formulated for dynamical problems as $D_{t} T+\operatorname{Div} X=0$ being $T$ the conserved density and $X$ the associated flux and the integration of the conserved density providing a constant of motion of the system, being the analogous expression for non-ordinary systems

$$
D_{t}(\rho)+D_{x}(\sigma)=0
$$

Generic densities being total $x$-derivatives satisfy this expression and are called trivial conservation laws and define equivalent densities as those differing in a total derivative. For evolution equations vanishing of the variational derivative operator $\frac{\delta}{\delta u}=\sum_{i=0}^{\infty}\left(-D_{x}\right)^{i} \frac{\partial}{\partial_{u_{i}}}$ provides a characterizations of total derivatives by

$$
\frac{\delta F}{\delta u}=0 \Longleftrightarrow F \in \operatorname{Im} D_{x}
$$

which will be of crucial importance in the definition and calculus with conservation laws.

Noether's first theorem associates a conservation law for the EulerLagrange equations with every one-parameter symmetry group of a variational problem. For instance, translation invariance leads to conservation of linear momentum, rotation invariance leads to conservation of angular momentum, and time translation invariance leads to conservation of energy. Noether's second theorem, of application in relativity and gauge theories, produces dependencies among the Euler-Lagrange equations arising from infinite-dimensional variational symmetry groups.

Conservation laws coming from formal (versus generalized) symmetries do not have any relationship to Noether's theorems, specially for single evolution equations. However, the results derived from the interesting properties of the formal symmetries set, as described in 1.8 provide a very fruitful framework for occurrence of conservation laws. We have seen how the rank $k$ formal symmetry condition leads to integrability constrains consisting of relations between the differential function defining the equation $K[u]$ and the coefficients of the generic formal symmetry $\mathscr{L}$. These relations have the additional advantage of letting the solvability conditions of the symmetry condition equation 1.7 .4 being recursively written and when leading to conservation laws, these provide the summary of the integrability constrains.

\subsection{CANONICAL CONSERVATION LAWS AS INTEGRABILITY CON- DITIONS}

The following results hold based on analysis conditions imposed by the existence of canonical conservation laws. We follow Sokolov and Shabat [45]. 
Any formal symmetry of rank $k \geq n$ of an n-th order evolution equation $(n \geq 2)$ provides $k-n$ canonical conserved densities

$$
\varrho_{j} \quad j=-1, \ldots, k-n-2
$$

and conversely if an evolution equation admits $k-n$ canonical conserved densities, then it has a formal symmetry of rank $k$.

Theorem 6. If $\mathscr{L}$ is a formal symmetry of the system $u_{t}=F[u]$ of degree 1 and rank $k>n$, then the $k-n$ canonical conservation laws

$$
D_{t} \rho_{i}=D_{x} \sigma_{i}, \quad i=-1, \ldots, k-n-2
$$

with

$$
\rho_{i}=\left\{\begin{array}{ll}
\operatorname{res} L^{i} & i \neq 0 \\
\operatorname{reslog} L & i=0
\end{array}, \quad \sigma_{i}= \begin{cases}D_{x}^{-1} \operatorname{res}\left[\mathrm{D}_{F}, L\right] & i \neq 0 \\
D_{x}^{-1} \operatorname{res}\left[\mathrm{D}_{F} L^{-1}, L\right] & i=0\end{cases}\right.
$$

are satisfied and are local ${ }^{1}$.

The above considerations can be extended with regard to the equation order, $n$, as follows. Given a generic first order formal symmetry $\mathscr{D}$ of rank $k \geq n+2$, then the residues of the first $k-n$ powers of $\mathscr{D}$

$$
\rho_{j}=\operatorname{Res} \mathscr{D}^{j}, j=-1, \ldots, k-n-2
$$

are conserved densities. This result provides a sequence of conservation laws in the form

$$
D_{t}\left(\rho_{j}\right)+D_{x}\left(\left[\mathscr{D}^{j}, \mathrm{D}_{K}\right]\right)=0
$$

which are not guaranteed to be non-trivial. When the rank of the generic first order symmetry is at least $n+2$, then

$$
\varrho_{-1}=\frac{1}{L_{1}}, \text { and } \varrho_{0}=\frac{L_{1}}{L_{0}}
$$

are conserved densities. If the formal symmetry has rank $n+1$, then just $Q_{-1}$ is conserved.

These type of integrability conditions in the form of local conservation laws are crucial in the symmetry approach to classification of integrable equations, not only for scalar evolution equation but also for non evolutionary equations, and non scalar (vector) and matrix equations. This formulation also turns to be very useful for the integrability and classification of generic integrable systems not restricted to PDEs nor evolution equations. It provides explicit integrability conditions also for ODEs, differential-difference equations, Lagrangian systems, etc. In fact these integrability conditions are usually given in the integrable systems literature by the canonical conservation laws listed as canonical densities. These encode the equation's integrability information and are the starting point for the classification of integrable equations described in the next section.

1 Locality means that they are differential functions of finite order 


\subsection{CLASSIFICATION METHOD}

As we have set in the preceding sections our definition of an equation's integrability is by the existence of infinitely many generalized symmetries of arbitrarily high order and this is the base classification criterium in the set of systems of differential equations, which will be partitioned in classes by the equivalence criterium of two integrable equations having the same symmetries being assigned to the same class. In this sense equivalence through contact transformations can be conveniently applied in the process and we will describe it from a computational point of view in chapter 3 , while devoting this chapter to show how formal calculus and its use in the theory of Shabat's (Mikhailov, Shabat, and Sokolov [26]) of formal symmetries provides a method for determining the integrability of generic evolution equations by setting the conditions for the possession of formal symmetries. In this chapter we show such approach in detail for second and third order integrable evolution equations.

By "generic evolution equations" above we mean the functional form of a family of equations expressed by an arbitrary function. Properties as order, linearity, dependence on spatial a temporal variables and number of dependent and independent variables and others are used as a means of setting equations types. Given an election of these, the formulae for symmetry conditions given above in this chapter are then applied. Integrability conditions as described in 1.8 can be calculated and used as necessary conditions to integrability for a more restricted type of equations.

The computation of the exact form of the equations, follows from the integration of the obstructions to integrability resulting from the expansion of the symmetry condition and leading to these exact forms in groups of equivalent equations (modulo a contact transformation) or integrable equations classes as the final output of this procedure.

In this chapter we describe this process for several types of evolution equations from the following theorem and lemma taken from Olver [34] which apply the FRO normalization described in section 1.8.

Theorem 7. If an evolution equation of order $n \geq 2$ possesses a nonconstant formal symmetry of rank $k$, then it has a first order formal symmetry of rank $k$. Conversely, if

$$
\mathscr{D}=Q_{1} D_{x}+Q_{0}+Q_{-1} D_{x}^{-1}+\cdots
$$

is a first order pseudo-differential operator which is a formal symmetry of rank $k$, then every formal symmetry of rank $k$ has the form

$$
\widetilde{\mathscr{D}}=c_{m} \mathscr{D}^{m}+c_{m-1} \mathscr{D}^{m-1}+\cdots+c_{m-k+2} \mathscr{D}^{m-k+2}+\mathscr{E}
$$

where $c_{m}, c_{m-1}, \ldots, c_{m-k+2}$ are arbitrary constant and $\mathscr{E}$ is any pseudo-differential operator of order at most $m-k+1$. 
The following lemma can be obtained from the form of the symmetry condition and the Fréchet linearization of the evolution equation

Lemma 8. The leading coefficient of an $n-t h$ order formal symmetry of rank $k \geq 2$ of an $n$-th order evolution equation $u_{t}=K[u]$ where $n \geq 2$ has the form

$$
c\left(K_{u_{n}}\right)^{m / n}
$$

for some constant $c \neq 0$.

This lemma follows from the first term of the expansion of the integrability condition $\mathscr{R}_{t} \doteq\left[\mathrm{D}_{K}, \mathscr{R}\right]$ and it is closely related to the existence of conservation laws and canonical densities introduced in 1.8 , as shown by the examples in this chapter.

\subsection{SECOND ORDER EVOLUTION EQUATIONS}

1.12.1 Equations of the form $u_{t}=u_{x x}+K\left[x, u, u_{x}\right]$

Following 1.7, the first step is to calculate the Fréchet derivative of the r.h.s. $u_{x x}+K\left(x, u, u_{x x}\right)$ as

$$
\begin{aligned}
\mathrm{D}_{K} & =\left(\partial_{u}+\partial_{u_{x}} D_{x}+\partial u_{x x} D_{x}^{2}\right)(K) \\
& =D_{x}^{2}+K_{u_{x}} D_{x}+K_{u}
\end{aligned}
$$

then, a first order with leading coefficient unity can be generally (see lemma 3) set as a FRO of this equation in the normalized form

$$
\mathscr{L}=D_{x}+Q_{0}+Q_{1} D_{x}^{-1}+Q_{2} D_{x}^{-2}+\cdots
$$

where $Q_{0}, Q_{1}, Q_{2}, \ldots$ are to be determined so as to satisfy the formal symmetry conditions. In order that $\mathscr{L}$ be a formal symmetry of rank $k$, the formal pseudo-differential operator

$$
\mathscr{E}=\mathbf{v}_{K}[[\mathscr{D}]]=(\mathscr{L})_{t}+\left[\mathscr{L}, \mathrm{D}_{K}\right]
$$

must vanish and must have order at most $n+m-k=3-k$ as per 1.7.5, and the setting of the leading coefficient of $\mathscr{L}$ to 1 implies that it is already a formal symmetry of rank $k=2$, so $\mathscr{E}$ has order at most 1 . 


$$
\begin{aligned}
\mathscr{E} & =\left(Q_{0}\right)_{t}+\left(Q_{1}\right)_{t} D_{x}^{-1}+\left(Q_{2}\right)_{t} D_{x}^{-2}+\cdots \\
& +\left(D_{x}+Q_{0}+Q_{1} D_{x}^{-1}+Q_{2} D_{x}^{-2}+\cdots\right)\left(D_{x}^{2}+K_{u_{x}} D_{x}+K_{u}\right) \\
& -\left(D_{x}^{2}+K_{u_{x}} D_{x}+K_{u}\right)\left(D_{x}+Q_{0}+Q_{1} D_{x}^{-1}+Q_{2} D_{x}^{-2}+\cdots\right) \\
& =Q_{0 t}+Q_{1 t} D_{x}^{-1}+Q_{2 t} D_{x}^{-2}+\cdots \\
& +D_{x}^{3}+\left(K_{u_{x}}\right)_{x} D_{x}+\left(K_{u}\right)_{x} \\
& +Q_{0} D_{x}^{2}+Q_{0} K_{u_{x}} D_{x}+Q_{0} K_{u} \\
& +Q_{1} D_{x}+Q_{1} D_{x}^{-1} K_{u_{x}} D_{x}+Q_{1} D_{x}^{-1} K_{u} \\
& +Q_{2}+Q_{2} D_{x}^{-2} K_{u_{x}} D_{x}+Q_{2} D_{x}^{-2} K_{u}+\cdots \\
& -D_{x}^{3}-D_{x}^{2} Q_{0}-D_{x}^{2} Q_{1} D_{x}^{-1}-D_{x}^{2} Q_{2} D_{x}^{-2}-\cdots \\
& -K_{u_{x}} D_{x}^{2}-K_{u_{x}} D_{x} Q_{0}-K_{u_{x}} D_{x} Q_{1} D_{x}^{-1}-K_{u_{x}} D_{x} Q_{2} D_{x}^{-2}-\cdots \\
& -K_{u} D_{x}-K_{u} Q_{0}-K_{u} Q_{1} D_{x}^{-1}-K_{u} Q_{2} D_{x}^{-2}-\cdots
\end{aligned}
$$

Requiring that the successive powers $D_{x}^{(i)}$ in $\mathscr{E}$ (starting in $i=1$ as justified above) to vanish will impose a series of stringent conditions on the operator and eventually in the equation itself that are necessary for the existence of a formal symmetry of progressively higher and higher orders (this is the equation being integrable as per our definition). After the Leibniz expansion of the terms in the Lie bracket above we can proceed to gathering of coefficients resulting in the following obstructions to integrability, equations which determine the functional form of $K[u]$.

- From the coefficient of $D_{x}$ in $\mathscr{E}$,

$$
\left(K_{u_{x}}\right)_{x}-2 D_{x} Q_{0}=0
$$

being the first integrability obstruction found, and leading to $Q_{0}=\frac{1}{2} K_{u_{x}}$ in order to have $\mathscr{L}$ as formal symmetry of rank 3 .

- Now, the coefficient of $D_{x}^{0}$ in $\mathscr{E}$ must equal 1 (in order to have a formal symmetry of rank 4 )

$$
Q_{0 t}+\left(K_{u}\right)_{x}-2\left(Q_{1}\right)_{x}-K_{u_{x}}\left(Q_{0}\right)_{x}=1
$$

which leads to the restriction of certain expression being the image of a total derivative, for which

$$
K_{u_{x} u_{x} u_{x}}=0
$$

results in $K\left(x, u, u_{x}\right)=\alpha(x, u) u_{x}^{2}+\beta(x, u) u_{x}+\gamma(x, u)$.

- The term in $u_{x}^{2}$ can be neglected after a contact transformation, resulting in our initial equation $u_{t}=u_{x x}+K\left[x, u, u_{x}\right]$ having quasi-linear form also in $u_{x}$

$$
u_{t}=u_{x x}+\beta(x, u) u_{x}+\gamma(x, u)
$$


with $\beta(x, u)=u$ and $\gamma(x, u)=\gamma(x)$ as consequences of the differential functions in the formal symmetry condition expressions being total derivatives. The resulting integrable equation is the Burgers' equation

$$
u_{t}=u_{x x}+u u_{x}+\gamma(x)
$$

- The simplification in the latter step results as a consequence of the formal symmetry property 1.12.1 being unaffected by changes of variables. These changes are in the class of first order contact transformations and allow us to find the solutions to the restrictions imposed by the formal symmetry condition. These restrictions are usually expressions that must lie in $\operatorname{Im} D_{x}$ or that must be total derivatives. These two techniques are the fundamental calculus techniques employed in the integrable equations classification process and are further analyzed in chapter 3 .

1.12.2 Sketch of the formal symmetry method applied to the classification of second order integrable equations.

Given a generic expression of a second order evolution equation

$$
u_{t}=K\left[x, u, u_{x}, u_{x x}\right]
$$

- The Fréchet derivative of this equation is

$$
\mathrm{D}_{\mathrm{K}}=K_{u_{x x}} D_{x}^{2}+K_{u_{x}} D_{x}+K_{u}
$$

- Let $\mathscr{D}$ be a generic formal symmetry of first order

$$
\mathscr{D}=L_{1} D_{x}+L_{0}+L_{-1} D_{x}^{-1}+L_{-2} D_{x}^{-2}+\cdots
$$

with $L_{i}$ differential functions satisfying the rank $k$ formal symmetry conditions. Lemma 8 can help in finding $L_{1}$.

- The symmetry condition expressed in formal pseudo-differential operators 1.7.4 expands by applying the product rules for pseudodifferential operators given in section 1.7 . 


$$
\begin{aligned}
\mathscr{E}= & \mathscr{D}_{t}+\left[\mathscr{D}_{,} \mathrm{D}_{\mathrm{K}}\right] \\
= & D_{t}\left(L_{1} D_{x}+L_{0}+L_{-1} D_{x}^{-1}+L_{-2} D_{x}^{-2}+\ldots\right) \\
& +\left(L_{1} D_{x}+L_{0}+L_{-1} D_{x}^{-1}+L_{-2} D_{x}^{-2}+\ldots\right)\left(K_{u_{x x}} D_{x}^{2}+K_{u_{x}} D_{x}+K_{u}\right) \\
& -\left(K_{u_{x x}} D_{x}^{2}+K_{u_{x}} D_{x}+K_{u}\right)\left(L_{1} D_{x}+L_{0}+L_{-1} D_{x}^{-1}+L_{-2} D_{x}^{-2}+\ldots\right) \\
= & D_{t}\left(L_{1}\right) D_{x}+D_{t}\left(L_{0}\right)+D_{t}\left(L_{-1}\right) D_{x}^{-1} \\
& +L_{1} K_{u_{x x}} D_{x}^{3}+L_{1} D_{x}\left(K_{u_{x x}}\right) D_{x}^{2}+L_{1} K_{u_{x}} D_{x}^{2}+L_{1} D_{x}\left(K_{u_{x}}\right) D_{x}+L_{1} K_{u} D_{x}+L_{1} D_{x}\left(K_{u}\right) \\
& +L_{0} K_{u_{x x}} D_{x}^{2}+L_{0} K_{u_{x}} D_{x}+L_{0} K_{u} \\
& +L_{-1} K_{u_{x x}} D_{x}-L_{-1} D_{x}\left(K_{u_{x x}}\right)+L_{-1} D_{x}^{2}\left(K_{u_{x x}}\right) D^{-1}+L_{-1} K_{u_{x}}-L_{-1} D_{x}\left(K_{u_{x}}\right) D^{-1} \\
& +L_{-1} K_{u} D_{x}^{-1}-L_{-1} D_{x}\left(K_{u}\right) D_{x}^{-2} \\
& +L_{-2} K_{u_{x x}}+L_{-2} K_{u_{x}} D_{x}^{-1}+L_{-2} K_{u} D_{x}^{-2} \\
& -K_{u_{x x}} L_{1} D_{x}^{3}-2 K_{u_{x x}} D_{x}\left(L_{1}\right) D_{x}^{2}-K_{u_{x x}} D_{x}^{2}\left(L_{1}\right) D_{x}-K_{u_{x x}} L_{0} D_{x}^{2}-2 K_{u_{x x}} D_{x}\left(L_{0}\right) D_{x}-K_{u_{x x}} D_{x}^{2}\left(L_{0}\right) \\
& -K_{u_{x x}} L_{-1} D_{x}-2 K_{u_{x x}} D_{x}\left(L_{-1}\right)-K_{u_{x x}} D_{x}^{2}\left(L_{-1}\right) D_{x}^{-1} \\
& -K_{u_{x}} L_{1} D_{x}^{2}-K_{u_{x}} D_{x}\left(L_{1}\right) D_{x}-K_{u_{x}} L_{0} D_{x}-K_{u_{x}} D_{x}\left(L_{0}\right)-K_{u_{x}} L_{-1}-K_{u_{x}} L_{-2} D_{x}^{-1} \\
& -K_{u} L_{1} D_{x}-K_{u} L_{0}-K_{u} L_{-1} D_{x}^{-1}-K_{u} L_{-2} D_{x}^{-2}
\end{aligned}
$$

- Gathering coefficients in each term of $\mathscr{E}$ we obtain how the formal symmetry coefficients are related to the evolution equation: $D_{x}^{3}$

$$
L_{1} K_{u_{x x}}-K_{u_{x x}} L_{1}=0
$$

$D_{x}^{2}:$

$$
\begin{aligned}
L_{1} D_{x}\left(K_{u_{x x}}\right)+L_{1} K_{u_{x}}+L_{0} K_{u_{x x}} & \\
-2 K_{u_{x x}} D\left(L_{1}\right)-K_{u_{x x}} L_{0}-K_{u_{x}} L_{1} & =L_{1} D_{x}\left(K_{u_{x x}}\right)-2 K_{u_{x x}} D_{x}\left(L_{1}\right) \\
L_{1} D_{x}\left(K_{u_{x x}}\right) & =2 K_{u_{x x}} D_{x}\left(L_{1}\right) \\
L_{1} & =\left(K_{u_{x x}}\right)^{1 / 2}
\end{aligned}
$$

$D_{x}:$

$$
D_{t}\left(L_{1}\right)=K_{u_{x x}} D_{x}^{2}\left(L_{1}\right)+2 K_{u_{x x}} D_{x}\left(L_{0}\right)+K_{u_{x}} D_{x}\left(L_{1}\right)-L_{1} D_{x}\left(K_{u_{x}}\right)
$$

where replacing the value found for $L_{1}$

$D_{t}\left(K_{u_{x x}}\right)^{1 / 2}=K_{u_{x x}} D_{x}^{2}\left(K_{u_{x x}}^{1 / 2}\right)+2 K_{u_{x x}} D_{x}\left(L_{0}\right)+K_{u_{x}} D_{x}\left(K_{u_{x x}}^{1 / 2}\right)-L_{1} D_{x}\left(K_{u_{x}}\right)$

where in order to find $L_{0}$ the total derivative $D_{x}$ needs to be inverted

$$
D_{t}\left(\left(K_{u_{x x}}\right)^{1 / 2}\right)=D_{x}\left(-D_{x} L_{1}-2 L_{0}+K_{u_{x}}\left(K_{u_{x x}}\right)^{-1 / 2}\right)
$$

- resulting in the integrability conditions taking the form of a conservation law, therefore requiring

$$
\frac{K_{u_{x}}}{K_{u_{x x}}} \in \operatorname{Im} D_{x}
$$

or being a total $x$-derivative. In this way we find a functional expression of the relation depicted in section 1.9 between integrable equations (restricted to evolutionary second order in this example) and conservation laws. 


\subsubsection{Classification theorem}

The complete analysis in above example combined with equivalence under first order contact transformations leads to the full classification of all second order evolution equations, which is summarized in the following theorem.

Theorem 9. Svinolupov [47]

Every second order evolution equation 1.12 .2 admitting a formal symmetry of rank 5 or more is integrable and is equivalent, under a contact transformation, to one of the following:

$$
\begin{aligned}
& u_{t}=u_{x x}+q(x) u \\
& u_{t}=u_{x x}+u u_{x}+h(x) \\
& u_{t}=\left(u^{-2} u_{x}+\alpha x u+\beta u\right)_{x} \\
& u_{t}=\left(u^{-2} u_{x}\right)_{x}+1
\end{aligned}
$$

\subsection{HIGHER ORDER EVOLUTION EQUATIONS}

Classification results along with explicit form of canonical densities for third and fifth order evolution equations can be found in Heredero [15].

Svinolupov and Sokolov [48] studied quasi-linear equations

$$
u_{t}=u_{x x x}+f\left(u, u_{x}, u_{x x}\right)
$$

being equivalent, modulo point transformations to the following list of integrable equations

$$
\begin{array}{cc}
u_{t}=u_{x x x}+\left(\alpha u^{2}+\beta u+\gamma\right) u_{x} & \mathrm{KdV} \\
u_{t}=u_{x x x}+\alpha u_{x}^{3}+\beta u_{x}^{2}+\gamma u_{x}+\delta & \text { Potential KdV } \\
u_{t}=u_{x x x}-\frac{1}{8} u_{x}^{3}+\left(\alpha e^{u}+\beta e^{-u}+\gamma\right) u_{x} & \text { Calogero-Degasperis } \\
u_{t}=u_{x x x}-\frac{3}{2} \frac{u_{x} u_{x x}^{2}}{u_{x}^{2}+1}-\frac{3}{2} \wp(u)\left(u_{x}^{3}+u_{x}\right)+\gamma u_{x} & \text { with }\left(\wp^{\prime}\right)^{2}=4 \wp^{2}+\alpha \wp+\beta
\end{array}
$$

For generic order 3 evolution equations

$$
u_{t}=F\left(x, u, u_{x}, u_{x x}, u_{x x x}\right)
$$


the formal symmetry method, applied in the procedure presented in section 1.12, leads to a series of integrability conditions imposing the following canonical densities

$$
\begin{aligned}
\rho_{0} & =\left(\frac{\partial F}{\partial u_{x x x}}\right)^{-\frac{1}{3}} \\
\rho_{1} & =\rho_{0}^{3} \frac{\partial F}{\partial u_{x x}} \\
\rho_{2} & =D\left(2 \rho_{0}^{-2} u_{x}+\rho_{0}^{2} \frac{\partial F}{\partial u_{x x}}\right)+\rho_{0}^{-3}\left(D \rho_{0}\right)^{2}+\frac{1}{3} \rho_{0}^{5}\left(\frac{\partial F}{\partial u_{x x}}\right)^{2} \\
& +\rho_{0}\left(D \rho_{0}\right) \frac{\partial F}{\partial u_{x x}}-\rho_{0}^{2} \frac{\partial F}{\partial u_{x}}+\rho_{0} \sigma_{0} \\
\rho_{3} & =-\frac{1}{3}\left(D^{2} \rho_{0}\right) \frac{\partial F}{\partial u_{x x}}-\left(D \rho_{0}\right) \frac{\partial F}{\partial u_{x}}+\rho_{0} \frac{\partial F}{\partial u}+\rho_{0}^{-1}\left(D \rho_{0}\right)^{2} \frac{\partial F}{\partial u_{x x}} \\
& -\frac{1}{3} \rho_{0}^{4} \frac{\partial F}{\partial u_{x}} \frac{\partial F}{\partial u_{x x}}+\frac{1}{3} \rho_{0}^{3}\left(D \rho_{0}\right)\left(\frac{\partial F}{\partial u_{x x}}\right)^{2} \\
& +\frac{2}{27} \rho_{0}^{7}\left(\frac{\partial F}{\partial u_{x x}}\right)^{3}+\frac{1}{3} \rho_{0} \sigma_{1} \\
\rho_{4} & =\rho_{0} \sigma_{2}-\rho_{2} \sigma_{0}
\end{aligned}
$$

Taking these canonical conservation laws as the integrability conditions for equations of the type 1.13.1, Ibragimov and Shabat [18] stated the following classification theorem:

Theorem 10. Any third order integrable evolution equation of the form 1.13.1 belongs to one of the following families

$$
\begin{aligned}
& u_{t}=a u_{x x x}+b \\
& u_{t}=\frac{1}{\left(a u_{x x x}+b\right)^{2}}+c \\
& u_{t}=\frac{2 a u_{x x x}+b}{\sqrt{a u_{x x x}^{2}+b u_{x x x}+c}}+d
\end{aligned}
$$

with $b^{2}-4 a c \neq 0$, and $a, b, c$ depending on $x, u, u_{x}, u_{x x}$.

\subsection{JET CALCULUS AND SYMMETRIES}

In this section we set the notation and the relevant methods for all calculations performed in the following chapters.

The generic formulations employed in the preceding sections are particularized with $p=2$ for $(1+1)$ systems in one spatial $x$ and one temporal $t$ variables. This notation covers evolution and nonevolutionary systems introduced in section 2.2, and for all of the defined objects we have:

- derivatives of a function $u=u(x, t)$ up to order $m$

$$
u_{J}^{(m)}=p^{(m)} u(x, t)=\left(u ; u_{x}, u_{t} ; u_{x x}, u_{x t}, u_{t t} ; u_{x x x}, u_{x x t}, u_{x t t}, u_{t t t}, \cdots\right)
$$


- $x$-derivatives are in the following sometimes denoted by

$$
u_{k}:=\frac{\partial^{k} u}{\partial x^{k}}
$$

i.e: $u_{3}=u_{x x x}=D_{x} u_{2}$, this is $D_{x} u_{k}=u_{k+1}$.

- Total derivatives with respect to $x^{i}$ as defined in the introduction (1.1.9)

$$
D_{i}=\frac{\partial}{\partial x^{i}}+\sum_{\alpha=1}^{q} \sum_{J} u_{J, i}^{\alpha} \frac{\partial}{\partial u_{J}^{\alpha}}
$$

will, for vector equations with solutions $\mathbf{u}=u^{\alpha}(x, t)$ take the form

$$
D_{x}=\frac{\partial}{\partial x}+\sum_{\alpha=1}^{q} u_{J, i}^{\alpha} \frac{\partial}{\partial u_{J}^{\alpha}}
$$

and denoted by $D:=D_{x}$, giving for $q=1$

$$
\begin{aligned}
& D=\partial_{x}+u_{1} \partial_{u}+u_{1 t} \partial_{u_{1}}+u_{2 t} \partial_{u_{2}}+\ldots+u_{n t} \partial_{u_{n}} \\
& D_{t}=\partial_{t}+u_{t} \partial_{u}+u_{1 t} \partial_{u_{t}}+u_{2 t} \partial_{u_{2}}+\ldots+u_{n t} \partial_{u_{n}}
\end{aligned}
$$

where $\partial_{f}:=\partial / \partial f$.

- Differential functions will be denoted with upper or lowercase letters $f, g, h, q, s, \ldots$ with generic (complex) equations given by $F[u]=0$ or $f=0$, and are

1. elements of corresponding finite order algebras

$$
\mathscr{F}_{n}[u]=\left\{F[u] ; \frac{\partial F}{\partial u_{n}} \neq 0\right\}
$$

such that $\mathscr{F}=\bigcup_{n=0}^{\infty} \mathscr{F}_{n}$,

2. defined and smooth on an open subset $M \subset X \times U^{(k)}$, and

3. understood to depend on a set of independent jet variables on $X \times U^{(k)}$

$$
\left\{x, t, u_{1}, u_{t}, u_{1 t}, u_{2}, u_{t t}, \cdots\right\}
$$


- Evolution equations in $(1+1)$ dimensions

$$
u_{t}=F\left(x, u, u_{1}, \cdots, u_{m}\right)
$$

can be represented as two infinite-dimension compatible dynamical systems or generalized fields in $J^{\infty}$ with the independent variable set introduced above:

$$
\begin{array}{rlrl}
(x)_{x}=1,(t)_{x}=0, \quad(t)_{t}=1, \quad(x)_{t}=0 & \\
\left(u_{i}\right)_{x}=u_{i+1} & & i=0,1, \ldots \\
\left(u_{i}\right)_{t}=u_{i t}=D^{i}(F) & & \text { (differential consequences) }
\end{array}
$$

- Local, infinitesimal, generalized symmetries of an equation with r.h.s. $F$ are differential functions $G$, satisfying the linearization

$$
G_{\tau}=\sum_{i=0}^{n} \partial_{i}(F) D^{i}(G)
$$

- For differential and pseudo-differential operators, the correspondent algebras $\mathscr{A}$ in the spaces of differential functions $\mathscr{F}$ are

$$
\begin{gathered}
\mathscr{F}(D)=\left\{\sum_{i=0}^{n} F_{i}[u] D^{i} ; F_{i} \in \mathscr{F} ; i=0,1,2, \cdots\right\} \\
\mathscr{F}((D))=\left\{\sum_{i=-\infty}^{n} F_{i}[u] D^{i} ; F_{i} \in \mathscr{F} ; i \in \mathbf{Z}\right\}
\end{gathered}
$$

as defined in section 1.7 and evolutionary vector fields as

$$
\mathscr{D}_{f}=\sum_{i=0}^{n}\left(D^{i} f\right) \partial_{u_{i}}, f \in \mathscr{F}
$$

commuting with total derivative $\left[D, \mathscr{D}_{f}\right]=0$.

- Fréchet derivatives will be differential operators in $\mathscr{F}$ denoted

$$
f^{*}:=\sum_{J} \frac{\partial f}{\partial u_{J}} D_{J}
$$

- For any $f, g \in \mathscr{A}$

$$
\left[\mathscr{D}_{f}, \mathscr{D}_{g}\right]=\mathscr{D}_{\mathscr{g}^{*}(f)-f^{*}(g)}
$$

- The following two properties have already been applied in preceding chapters to derive fundamental properties of equations in operators form

$$
\begin{gathered}
(f g)^{*}=f g^{*}+g f^{*} \\
(D(f))^{*}=D \cdot f^{*}=D\left(f^{*}\right)+f^{*} D \\
\left(\frac{d}{d t} f\right)^{*}=\frac{d}{d t}\left(f^{*}\right)+f^{*} F^{*}
\end{gathered}
$$


- Symmetry conditions for symmetries $Q$ of equation $H=0$ become

$$
\sum_{J} \frac{\partial H}{\partial u_{J}} D_{J} Q \doteq 0
$$

which after linearization, in differential operators form, simplifies to

$$
H^{*}(Q)=0
$$

- The conditions for local symmetries of a given evolutionary equation

$$
u_{t}=q\left(t, x, u, u_{1}, u_{2}, \cdots, u_{n}\right)
$$

are derived from the compatibility condition of the above equation with the flow generated by its evolutionary generalized ( $m$-th order) symmetries

$$
u_{\tau}=s\left(t, x, u, u_{1}, u_{2}, \cdots, u_{m}\right)
$$

which is

$$
u_{\tau t}-u_{t \tau}=D_{\tau} q-D_{t} s \doteq 0
$$

and by the chain rule above

$$
\begin{aligned}
q_{u} D_{\tau} u+q_{u_{1}} D_{\tau} u_{1}+\ldots+q_{u_{n}} D_{\tau} u_{n} & \doteq s_{t}+s_{u} u_{t}+\ldots+s_{u_{m}} D_{t} u_{m} \\
q_{u} s+q_{u_{1}} D_{x} s+\ldots+q_{u_{n}} D_{x}^{n} s & \doteq s_{t}+s_{u} q+\ldots+s_{u_{m}} D_{x}^{m} q
\end{aligned}
$$

- The second equation above derived from the differential consequences of $u_{t}=q$,

$$
\left(u_{i}\right)_{t}=D^{i}(q)
$$

and its linearization

$$
q^{*}=\sum_{i=0}^{n} \partial_{i}(q) D^{i}(g)
$$

we can rewrite $u_{t \tau}=s_{t}$ as

$$
q^{*}(s)-s_{t}-s^{*}(q)=0
$$

and by 1.14 .5

$$
[q, s]=s^{*}(q)-q^{*}(s)=s_{t}
$$

- After a second linearization by using the Fréchet derivative of the time derivative of the symmetry $s$ the equation above is equivalent to

$$
D_{t}\left(s^{*}\right)-[q, s]=s^{*}(q)-q^{*}(s)=0
$$

this is

$$
D_{t}\left(s^{*}\right) \doteq\left[q^{*}, s^{*}\right]
$$

which resembles the symmetry condition 1.8.1 obtained for symmetries of evolution equations, extended with the following characteristics 
- written in operator form (computationally much more efficient)

- for generalized non-evolutionary symmetries of equation $u_{t}=q$.

- order of a symmetry $s$, is given by the degree of differential operator $s^{*}$

- The same considerations about a formal symmetry satisfying the condition above apply regarding the orders of the

- differential ( $q^{*}$ representing the equation) and

- pseudo-differential $\left(s^{*}\right.$ representing a FRO)

operators involved:

$$
\begin{aligned}
& \# q^{*}=n \quad \ll \quad s^{*}=m \\
& \#\left[q^{*}, s^{*}\right]=n+m-1
\end{aligned}
$$



EXTENSIONS TO THE FORMAL SYMMETRY APPROACH

The symmetry approach refers to the formal calculus concepts and techniques, including those of integrable evolution equations classifications as described in the preceding chapter. Formal symmetries were developed in the work of Shabat and collaborators (1979-present) and published in the references such as Mikhailov, Shabat, and Yamilov [25] and Sokolov [44]. We have seen that the formulation of this method is based on the symmetry conditions for a generic system of differential equations from the principle of invariance of the system under the generalized (meaning the differential order not specified) prolonged action of prolonged fields in a way such that the symmetry conditions define new generalized vector fields, or symmetries, commuting (meaning having vanishing Lie bracket) with the flow originated by a given $n$-th order system of differential equations.

The formal symmetry conditions have their generic formulation for systems of 2 independent variables and $q$ dependent variables of which we have only specified one variable considered "spatial" and another "temporal". Section 1.4 introduced systems of $q$ evolution equations with $q$ dependent variables involved in the differential functions defining the r.h.s.s $K_{1}[\mathbf{u}], \cdots, K_{q}[\mathbf{u}]$ of $\Delta=\mathbf{u}_{t}-\mathbf{K}[\mathbf{u}]$. In subsequent sections the theory was developed for scalar evolution equations with $q=1$.

\section{I STATE OF THE ART OF THE SYMMETRY APPROACH TO INTE- GRABILITY}

A natural first step in extending of this theoretical framework was taken by formalizing its concepts and techniques to include systems of evolution equations with $q>1$. This work is formulated in Mikhailov, Shabat, and Yamilov [25] for the case of the highest order derivatives matrix having diagonal form with nonzero different eigenvalues.

The current state of the art of this extensions is the underlying theory for the results presented here and focuses on formalizing the symmetry approach to non evolutionary equations. Permitting $x t$ derivatives in r.h.s. of $\Delta$ takes the problem of finding formal symmetry conditions back to the point where we took advantage of the computational simplifications introduced in the preceding chapters and opens a new fork in the framework of formal symmetries to pseudodifferential structures in two derivations $D_{x}$ and $D_{t}$. 


\subsection{EXTENSIONS TO NON-EVOLUTIONARY EQUATIONS}

Widening the class of systems to those having $t$-derivatives of the dependent variable of higher order than one we obtain non-evolutionary systems. The normalized form of writing these equations is with l.h.s.s depending only on $t$-derivatives only and the r.h.s.s being differential functions depending on lower order $t$-derivatives or mixed $x t$-derivatives of lower order in $t$. We will deal in this thesis only with non-evolutionary systems of order 2 in $t$ of the form

$$
\begin{gathered}
u_{t t}=F\left(u, u_{x}, u_{x x}, \ldots, u_{n}, u_{t}, u_{x t}, u_{x x t}, \cdots, u_{m t}\right) \\
u_{i}=\frac{\partial^{i} u}{\partial x^{i}}, \quad u_{i t}=\frac{\partial^{i+1} u}{\partial x^{i} \partial t}
\end{gathered}
$$

We define the differential order of such systems as the pair $(n, m)$ with $n$ as the order on $x$-derivatives and $m$ the order in $x$ of $x t$-derivatives.

As generalizations to non-evolutionary equations we have Novikov and Wang [33] and Mikhailov, Novikov, and Wang [27], where general results were obtained using a perturbative symmetry approach in symbolic representation. The price to pay for such generality is that this representation, in principle, requires the equation to be polynomial and homogeneous. However, the use of other representations like those in $[27,33]$ allow for the introduction of some nonlocalities that appear in some important integrable systems like the Boussinesq equation.

The paper by R. Hernández Heredero, A. Shabat and V. Sokolov [16] describes how a new class of integrable PDE is found by extending the formal symmetry approach to order $(3,1)$ non-evolutionary equations. These type of equations feature characteristics related to integrability which makes the study of their symmetries very interesting for both testing known integrability conjectures (as the Painlevé test) and understanding integrability of this class of equations by finding all equations in the class with higher (or generalized) symmetries. Following the notation in the preceding chapters such equations can be generally written as

$$
u_{t t}=u_{x x x}+F\left(u, u_{x}, u_{t}, u_{x x}, u_{x t}\right)
$$

as order $(3,1)$ equations of the wider class 2.2.1 that were not included in preceding extensions of the formal symmetry approach, which were restricted to systems of evolution equations. The standard method of deriving necessary integrability conditions based on the residues of fractional powers of the FRO does not work for these equations and it has been necessary to develop a generalization of the methods of the symmetry approach.

The extended method of formal recursion operators and canonical conserved densities (1.8) is applied to non-evolutionary equations of order $(3,1)$, generalizing the main concepts of the formal symmetry 
approach and extending its reach to new types of equations. This is done by using a new definition of canonical densities. This results are summarized in the following theorem taken from Heredero, Shabat, and Sokolov [16].

Theorem 11. If equation 2.2.3 possesses an infinite sequence of higher symmetries of the form

$$
u_{\tau_{i}}=G_{i}\left(u, u_{x}, u_{x x}, \cdots, u_{r_{i}}, \cdots ; u_{t}, u_{x t}, \cdots, u_{s_{i} t}, \cdots\right)
$$

then there exists a formal recursion operator of the form

$$
\mathscr{R}=\left(l_{0}+l_{-1} D^{-1}+\cdots\right)+\left(D^{-1}+m_{-2} D^{-2}+\cdots\right) D_{t}
$$

where $l_{i}, m_{j}$ are some functions of the variables $u, u_{x}, u_{x x}, \ldots, u_{r}, \ldots ; u_{t}$, $u_{x t}, \ldots, u_{s t}, \ldots$.

Motivated by the study of Lagrangian systems, we will need to extend the symmetry approach method to non-evolutionary equations of order $(4,1)$ or even unspecified order $(n, m)$ 2.2.1:

$$
u_{t t}=F\left(u, u_{x}, u_{x x}, \ldots, u_{n}, u_{t}, u_{x t}, u_{x x t}, \cdots, u_{m t}\right) .
$$

Equations of this form have symmetries that can be expressed as

$$
u_{\tau}=G\left(u, u_{1}, u_{2}, \ldots, u_{r}, u_{t}, u_{1 t}, u_{2 t}, \ldots, u_{s t}\right) .
$$

The symmetry condition 1.6.4, $\mathrm{D}_{G}\left(u_{t t}-F\right) \doteq 0$, where the symbol $\doteq$ denotes equality in the solution space of the equation, is the compatibility conditions for the previous two equations. The symmetry condition written in this form seems not amenable to be treated by the standard tools of formal symmetry approach (i.e. characterizing formal recursion operators), mainly because it is not written in evolutionary form (there is an asymmetry between the form of the equation and of the symmetry). One could think of writing the equation, of order two in $t$, as a system of two evolution equations of order one in $t$, and use the theory in [25]. But this is not possible because the matrix of highest derivatives of the system turns out to be nondiagonalizable. The solution consists on considering the compatibility of the linearizations of equations 2.2.4 and 2.2.5

$$
\begin{aligned}
\phi_{t t} & =\left(A+B D_{t}\right) \phi \\
\phi_{\tau} & =\left(L+M D_{t}\right) \phi
\end{aligned}
$$

where $A, B, L, M$ are differential operators only in $D_{x}$ (we will detail them in the next Section). And now, indeed, writing the symmetry condition in evolutionary form as a matrix system of equations

$$
\begin{aligned}
\Phi_{t} & =\mathrm{D}_{F} \Phi \\
\Phi_{\tau} & =\mathrm{D}_{G} \Phi
\end{aligned}
$$


with

$$
\begin{aligned}
\Phi & =\left(\begin{array}{l}
\phi \\
\phi_{t}
\end{array}\right) \\
\mathrm{D}_{F} & =\left(\begin{array}{ll}
0 & 1 \\
A & B
\end{array}\right) \\
\mathrm{D}_{G} & =\left(\begin{array}{ll}
L & M \\
\hat{L} & \hat{M}
\end{array}\right) \\
\hat{L} & :=L_{t}+M A \\
\hat{M} & :=M_{t}+M B+L
\end{aligned}
$$

The compatibility conditions (cross-differentiation) then lead to

$$
D_{t}\left(\mathrm{D}_{G}\right)=\left[\mathrm{D}_{F}, \mathrm{D}_{G}\right]+D_{\tau}\left(\mathrm{D}_{F}\right)
$$

and taking into account the consideration about the order of each of these terms, as mentioned in 1.7 .3 and 1.7.4, we obtain the key matrix operator symmetry condition

$$
D_{t}(\mathbf{R}) \doteq\left[\mathrm{D}_{F}, \mathbf{R}\right]
$$

for integrability of 2.2.4 as the existence of a matrix formal recursion operator $\mathbf{R}$

$$
\mathrm{R}=\left(\begin{array}{cc}
L & M \\
\hat{L} & \hat{M}
\end{array}\right), \quad \hat{L}:=L_{t}+M A, \quad \hat{M}:=M_{t}+M B+L .
$$

Like in the case of scalar evolution equations, in 2.2.6 the operators $L$ and $M$ have to be taken as formal pseudodifferential series .

This condition yields the following system of relations between the differential operators in the equation $A, B$ and the pseudo-differential operators $L, M$ in its formal symmetries generated by matrix FRO $\mathbf{R}$

$$
\begin{gathered}
L_{t t}-B L_{t}+[L, A]+\left(2 M_{t}+[M, B]\right) A+M A_{t}=0 \\
M_{t t}-B M_{t}+[L, B]+\left(2 M_{t}+[M, B]\right) B+M B_{t}+2 L_{t}+[M, A]=0
\end{gathered}
$$

Further development of these conditions results in a series of theorems, proved in Quintero and Heredero [39], for the existence of formal recursion operators and integrability conditions in the form of local conservation laws. New ways of obtaining necessary integrability conditions need to be derived because the ones presented in the introduction (being the use of residues of powers of the formal recursion operator to obtain canonical conservation laws the most representative of the formal symmetries approach) do not work for nonevolutionary equations. Definition of canonical conservation laws and densities are generalized in order to adapt the formal symmetry approach to non-evolutionary equations. 
This theoretical framework is of recent development and represents the branch of the current state of the art which we employ in the approach for the classification of Lagrangian systems as an application of the extended symmetry approach to integrability. We will see in the next section how the extension of the symmetry approach techniques and concepts to equations of the form 2.2.4 is required to achieve new results in the subject of second order integrable Lagrangian systems classification.

\subsection{THE STRUCTURE OF THE SOLUTION SPACE OF THE EXTENDED SYMMETRY CONDITION FOR NON-EVOLUTIONARY EQUATIONS}

The extended symmetry approach derives its features from similar but more complicated (not yet fully formalized) properties to those introduced in section 1.8 for the evolutionary case. There we saw how FROs provide increasingly higher rank symmetries of a given equation which encode its integrability in the form of canonical conservation laws. Furthermore the theorems and lemmas there quoted provide a computational approach, based on the normalization of FROs, which was presented as a technical lemma summarizing the algebraic properties of the solution space of the formal symmetry condition equation 1.8.1. These properties are derived from the left and right product, power and roots of a formal symmetry of rank $k$ being a formal symmetry of rank $k$, therefore producing an algebraic structure of a non-commutative ring in the set of formal symmetries of a given equation.

Recent research in the analogous properties of matrix FROs for nonevolutionary equations shows how this algebraic structure is even richer than in the evolutionary case. The algebraic structure of space of solutions of the determining equations for a generic symmetry resembles the integrability properties of the equation. All of this properties are proved in Quintero and Heredero [39], and quoted below.

- The space of FROs has the structure of a non-commutative field.

- For non-evolutionary equations there are two derivations $D_{x}$ and $D_{t}$ acting on the solutions space and commuting with each other. This produces integrability conditions in the form of conservation laws just like in the evolutionary case. An additional operator $D_{\tau}$ permits linearization of generalized symmetries.

- Differential order has to be extended to be specified in pairs $(n, m)$ (as seen above in this chapter). Analogously the rank conditions are formulated for the spatial and temporal variables, in pairs $(r, s)$. Rank is conserved by all product operations and by linearization.

Unlike the evolution equations case, we don't have $D_{t}$ defined by the system itself. 
- There exist remarkable recursive relations between the coefficients of the normalized base of matrix FROs that makes this structure very rich from both the algebraic and the computational points of view.

- There is an isomorphism between the matrix formulation above and the scalar formulation we employ in the sequel.

- Allows extensions of the symmetry approach to integrability to non-evolutionary equations any differential order $(n, m)$

$$
u_{t t}=f\left(u, u_{x}, \ldots, u_{n} ; u_{t}, u_{x t}, \ldots, u_{m t}\right)
$$

where

$$
u_{t t}:=\frac{\partial^{2} u}{\partial t^{2}}, \quad u_{j t}:=\frac{\partial^{j+1} u}{\partial x^{j} \partial t}, \quad j \in \mathbf{N}, \quad \text { and } \quad u_{0 t}:=u_{t} .
$$

We consider, within the framework of differential algebra, a set $\mathfrak{H}$ of differential functions $h\left(u, u_{x}, \ldots, u_{k} ; u_{t}, u_{x t}, \ldots, u_{l t}\right)$ with two derivations $D:=D_{x}$ and $D_{t}$. If $\partial h / \partial u_{k} \neq 0$ and $\partial h / \partial u_{l t} \neq 0$ the pair $(k, l)$ will denote the differential order of $h$. The derivations $D$ and $D_{t}$ act over differential functions $h \in \mathfrak{H}$ as total derivatives

$$
\begin{gathered}
D h=\sum_{i=0}^{k} \frac{\partial h}{\partial u_{i}} u_{i+1}+\sum_{j=0}^{l} \frac{\partial h}{\partial u_{j t}} u_{j+1 t} \\
D_{t} h=\sum_{i=0}^{k} \frac{\partial h}{\partial u_{i}} u_{i+1 t}+\sum_{j=0}^{l} \frac{\partial h}{\partial u_{j t}} u_{j+1 t t} \doteq \sum_{i=0}^{k} \frac{\partial h}{\partial u_{i}} u_{i+1 t}+\sum_{j=0}^{s} \frac{\partial h}{\partial u_{j t}} D^{j+1} f
\end{gathered}
$$

where the symbol $\doteq$ denotes an equality that holds only in the solution space of 2.3.1, i.e. $u_{t t}$ and its $x$-derivatives $u_{j t t}$ are to be substituted by $f$ and its corresponding total derivatives.

Generalized Lie symmetries of 2.3.1 are of the form

$$
u_{\tau}=g\left(u, u_{x}, \ldots, u_{r} ; u_{t}, u_{x t}, \ldots, u_{s t}\right)
$$

with $g \in \mathfrak{H}$. A generalized symmetry $g$ provides an additional derivation $D_{\tau}$ in the differential algebra that commutes with $D$ and acts on a differential function $h$ as

$$
D_{\tau} h=\sum_{i=0}^{k} \frac{\partial h}{\partial u_{i}} D^{i} g+\sum_{j=0}^{l} \frac{\partial h}{\partial u_{j t}} D^{j} D_{t} g .
$$

We will use indistinctly the notations $D_{\tau} h=h_{\tau}=D_{\tau}(h)$.

The symmetry condition, i.e. the compatibility of 2.3 .1 and 2.3 .2 is then

$$
D_{\tau} f \doteq D_{t}^{2} g
$$

and it can be expressed in terms of the linearisation, or variational equation, of 2.3.1. We write this variational equation as

$$
D_{t}^{2} \phi=\left(A+B D_{t}\right) \phi
$$


where $\phi=\phi(x, t)$ is a new dependent variable, the variation, and

$$
\mathcal{F}:=A+B D_{t}
$$

is the linearisation operator of the differential function $f$, being $A$ and $B$ differential operators on $D$ only:

$$
\begin{aligned}
& A:=a_{n} D^{n}+a_{n-1} D^{n-1}+\cdots+a_{0}, \\
& B:=b_{m} D^{m}+b_{m-1} D^{m-1}+\cdots+b_{0}
\end{aligned}
$$

with

$$
a_{i}:=\frac{\partial^{i} f}{\partial u_{i}}, \quad b_{j}:=\frac{\partial f}{\partial u_{j t}} .
$$

The symmetry condition 2.3 .3 can now be written as

$$
D_{t}^{2} g \doteq\left(A+B D_{t}\right) g
$$

We are interested in equations that are integrable from the symmetry approach point of view, i.e. equations that admit an infinite number of symmetries 2.3.2 of arbitrarily high differential order $(r, s)$. To treat this problem, it is convenient to use an alternative way to write the symmetry condition. It consists in performing a second linearisation on 2.3.6 or, equivalently, considering the compatibility of the linearized versions of 2.3.1 and 2.3.2. The linearized version of 2.3.1 is 2.3 .4 , and that of 2.3 .2 is

$$
\phi_{\tau}=\left(L+M D_{t}\right) \phi,
$$

where $\mathcal{G}:=L+M D_{t}$ is the linearisation operator of the differential function $g$, i.e.

$$
L=l_{r} D^{r}+l_{r-1} D^{r-1}+\cdots+l_{0}, \quad M=m_{s} D^{s}+m_{s-1} D^{s-1}+\cdots+m_{0}
$$

are differential operators with

$$
l_{i}:=\frac{\partial g}{\partial u_{i}}, \quad m_{j}:=\frac{\partial g}{\partial u_{j t}} .
$$

Now the compatibility of equations 2.3.4 and 2.3.7 leads to symmetry conditions that can be written as equations over differential operators:

$$
\begin{aligned}
L_{t t}-B L_{t}+[L, A]+\left(2 M_{t}+[M, B]\right) A+M A_{t} & =A_{\tau} \\
M_{t t}-B M_{t}+[L, B]+\left(2 M_{t}+[M, B]\right) B+M B_{t} & \\
+2 L_{t}+[M, A] & =B_{\tau}
\end{aligned}
$$

In this formula we have defined the extension of a derivation $D_{\tau}$ (or $D_{t}, D_{x}$ ) to a differential operator $O=o_{k} D^{k}+o_{k-1} D^{k-1}+\cdots+o_{0}$ as

$$
O_{\tau}=D_{\tau}\left(o_{k}\right) D^{k}+D_{\tau}\left(o_{k-1}\right) D^{k-1}+\cdots+D_{\tau}\left(o_{0}\right) .
$$


Equations 2.3.8 have to be interpreted as the system of PDE's obtained equating every coefficient of $D^{j}$ in the lhs's to the corresponding coefficient in the rhs's. Equations 2.3 .8 can be used to find symmetries of a fixed differential order $(r, s)$. In this case, the operators $A$ and $B$ are determined by the equation, so they are data in 2.3.8, while the operators $L$ and $M$ are the unknowns. This procedure is completely equivalent to directly finding symmetries from 2.3.8. In the problem of finding integrable equations all four operators $A, B$ (associated to the equation 2.3.1) and $L, M$ (associated to the symmetry 2.3.2) are unknowns in 2.3.8. But the fact that we require the existence of symmetries of an arbitrarily high order, i.e. that the operators $L$ and $M$ have to be of an arbitrarily high degree in $D$, allows to simplify the problem. For an arbitrary pair of differential operators $L$ and $M$ of degrees $r$ and $s$, the left hand sides of 2.3.8 have generically degrees higher than $r$ and $s$. But the right hand sides are

$$
A_{\tau}=D_{\tau}\left(a_{n}\right) D^{n}+\cdots+D_{\tau}\left(a_{0}\right), \quad B_{\tau}=D_{\tau}\left(b_{m}\right) D^{m}+\cdots+D_{\tau}\left(b_{0}\right)
$$

and have generically a fixed degree of $n$ and $m$ respectively. If there are symmetries of higher order $r \gg n$ and $s \gg m$, the number of conditions to be met in the left hand sides increases when increasing $r$ and $s$, tending to be infinite in the arbitrarily high order case. The symmetry approach then proposes (see [16]) to consider the operator equations

$$
\begin{aligned}
L_{t t}-B L_{t}+[L, A]+\left(2 M_{t}+[M, B]\right) A+M A_{t} & =0 \\
M_{t t}-B M_{t}+[L, B]+\left(2 M_{t}+[M, B]\right) B+M B_{t} & \\
+2 L_{t}+[M, A] & =0
\end{aligned}
$$

In order to make this idea to work the operators $L$ and $M$ that solve 2.3.9 must be taken in the class of pseudo-differential operators instead of differential operators. Equations 2.3.I for which there exist nontrivial solutions to 2.3 .9 are deemed as integrable under the symmetry approach, because it is a necessary condition for having symmetries of arbitrarily high order.

The algebraic structure of the space of solutions $\mathcal{R}:=L+M D_{t}$ of 2.3 .9 is much richer than that of its non-linearized version. It is based on the following properties:

1. The product of two solutions, defined as

$$
\begin{gathered}
\left(L_{1}+M_{1} D_{t}\right) \cdot\left(L_{2}+M_{2} D_{t}\right)=L_{1} \cdot L_{2}+M_{1} \cdot\left(L_{2}\right)_{t}+M_{1} \cdot M_{2} \cdot A \\
+\left[L_{1} \cdot M_{2}+M_{1} \cdot L_{2}+M_{1} \cdot\left(M_{2}\right)_{t}+M_{1} \cdot M_{2} \cdot B\right] D_{t}
\end{gathered}
$$

is also a solution, a reflection of the fact that the set of generalized symmetries is a Lie algebra.

2. The identity operator $\mathcal{I}=1+0 D_{t}$ is always a solution. 
3. The inverse of a differential operator exists in the (non-commutative) field of pseudo-differential operators. We will consider operators

$$
\mathbf{R}=L+M D_{t}
$$

where $L$ and $M$ are pseudo-differential operators of degrees $r$ and $s$

$$
\begin{gathered}
L=l_{r} D^{r}+l_{r-1} D^{r-1}+\cdots+l_{0}+l_{-1} D^{-1}+\cdots, \\
M=m_{s} D^{s}+m_{s-1} D^{s-1}+\cdots+m_{0}+m_{-1} D^{-1}+\cdots
\end{gathered}
$$

being $l_{i}, m_{j}$ differential functions.

4. The product of pseudo-differential operators follows the standard integration by parts rule

$$
D^{-1} \cdot \mathcal{O}=\mathcal{O} D^{-1}-D^{-1} \cdot D(\mathcal{O}) D^{-1}=\sum_{i=0}^{\infty}(-1)^{i} D^{i}(\mathcal{O}) D^{-1-i}
$$

5. In the class of pseudo-differential operators every nonzero operator $\mathcal{R}$ has a unique inverse $\mathcal{R}^{-1}$ and, if $\mathcal{R}$ is a solution of 2.3.9 then $\mathcal{R}^{-1}$ is also a solution. These solutions $\mathcal{R}$ are called formal recursion operators (briefly FROs as introduced in previous chapters) of the given differential equation 2.3.1. This name is justified because they satisfy the equation

$$
\mathcal{H}\left(L+M D_{t}\right) \doteq\left(\bar{L}+M D_{t}\right) \mathcal{H}
$$

where $\mathcal{H}:=D_{t}^{2}-A-B D_{t}$ is the linearisation operator of 2.3.I and $\bar{L}:=L+2 M_{t}+[M, B]$.

The proofs of the assertions in the previous paragraph are easily done using an alternative, matrix formulation of the problem. It is based on representing the differential equation 2.3.1 as a first order system

$$
\begin{aligned}
& u_{t}=v \\
& v_{t}=f\left(u, u_{x}, \ldots, u_{r}, v, v_{x}, \ldots, v_{s}\right)
\end{aligned}
$$

and performing the double linearisation process described in the previous section. The variational equations of 2.3.14 can be written in a matrix form as

$$
\left(\begin{array}{l}
\phi \\
\psi
\end{array}\right)_{t}=\mathbf{F}\left(\begin{array}{l}
\phi \\
\psi
\end{array}\right) \quad \text { with } \quad \mathbf{F}:=\left(\begin{array}{ll}
0 & 1 \\
A & B
\end{array}\right)
$$

and the variational equations of 2.3 .17 as

$$
\begin{aligned}
& \qquad\left(\begin{array}{l}
\phi \\
\psi
\end{array}\right)_{\tau}=\mathbf{R}\left(\begin{array}{l}
\phi \\
\psi
\end{array}\right) \text { with } \quad \mathbf{R}:=\left(\begin{array}{cc}
L & M \\
\widehat{L} & \widehat{M}
\end{array}\right) \\
& \text { and } \widehat{L}:=L_{t}+M A, \quad \widehat{M}:=M_{t}+M B+L
\end{aligned}
$$


The commutation of these two equations gives rise to the usual expression for a recursion operator in matrix form

$$
\mathbf{R}_{t}=[\mathbf{F}, \mathbf{R}]
$$

Using this equation it is straightforward to prove the following basic features of the set $\mathfrak{F}$ of formal matrix recursion operators of 2.3.1, that is, the space of solutions $\mathbf{R}$ of 2.3.16 given a fixed equation 2.3.1, i.e. a fixed $\mathbf{F}$.

Proposition 12. Let $\mathfrak{F}$ the set of formal matrix recursion operators of equation 2.3.1, i.e. the set of solutions $\mathbf{R}$ to equation 2.3.16. Then

1. The set $\mathfrak{F}$ is a linear space over the constants.

2. The product of two FRO's $\mathbf{R}_{1}, \mathbf{R}_{2} \in \mathfrak{F}$ is a $F R O \mathbf{R}_{1} \cdot \mathbf{R}_{2} \in \mathfrak{F}$.

3. For any $F R O \mathbf{R} \in \mathfrak{F}, \mathbf{R} \neq 0$ there exists an inverse $F R O \mathbf{R}^{-1} \in \mathfrak{F}$.

Thus $\mathfrak{F}$ is a non-commutative field.

The addition, product and inverses of matrix FRO's correspond univocally to the same operations in scalar FROs, and equation 2.3.16 is completely equivalent to equations 2.3.9. Thus there is an isomorphism between scalar and matrix FROs. Every formal (scalar) recursion operator $\mathbf{R}=L+M D_{t}$ has a unique associated formal matrix recursion operator $\mathbf{R}$ that is a solution of 2.3.16 and has the form given in 2.3.15. We will use from now on only scalar recursion operators $\mathbf{R}$, because they present some computational advantages.

There is a relation between higher order generalized symmetries and formal recursion operators. If a generalized symmetry $g$ has a sufficiently high order, its linearisation $\mathcal{G}=L+M D_{t}$ annihilates many terms in the equations of a fro 2.3.9 and it gives the leading behavior of a fro of the same order. Thus, although a generalized symmetry does not generate a full formal recursion operator because the rhs's of 42

$$
u_{\tau}=g\left(u, u_{x}, \ldots, u_{r} ; u_{t}, u_{x t}, \ldots, u_{s t}\right)
$$

are not zero, it partially solves equations 2.3.9. This fact is formalized by the following definition, where $\operatorname{deg}(\mathcal{O})$ denotes the degree of a pseudo-differential operator $\mathcal{O}$, i.e. its maximum power in $D$.

Definition 13. The pseudo-differential operator

$$
\mathcal{R}=L+M D_{t}
$$

with $\operatorname{deg}(L)=r, \operatorname{deg}(M)=s$ is a formal symmetry (or approximate symmetry) of rank $(k, l)$, of equation 2.3.1, if the left hand sides of 2.3 .9 are of degree at most $r+n-k$ and $s+m-l$ respectively. 
The next result follows.

Proposition 14. The linearisation $\mathcal{G}=L+M D_{t}$ of a symmetry 2.3.17 of equation 2.3.1 is a formal symmetry of rank $(r, s)$.

The space of formal symmetries of a given rank has the same properties than the space of FRO's. It is a linear space over the constants, the product of two formal symmetries of the same rank is a formal symmetry of the same rank, and the inverse of a formal symmetry is a formal symmetry of the same rank.

Proposition 15. If an equation 2.3.1 admits infinite higher symmetries of arbitrarily high order, then it admits a non-trivial formal recursion operator $\mathbf{R}=L+M D_{t}$, i.e. there exist nontrivial local solutions 2.3.11 to 2.3.9.

We write the generic formal recursion operator as a pseudo-differential operator 2.3.10. For computational reasons and without loss of generality we initially set $\operatorname{deg}(L)=r=k$ and $\operatorname{deg} M=s=k-1$, with $k \in \mathbf{Z}$, writing

$$
\begin{gathered}
L=l_{k} D^{k}+l_{k-1} D^{k-1}+\cdots+l_{i} D^{i}+\cdots \\
M=m_{k-1} D^{k-1}+m_{k-2} D^{k-2}+\cdots+m_{j} D^{j}+\cdots .
\end{gathered}
$$

The first integrability conditions for 2.3.I with $n=4$ and $m=1$ are the following.

- The highest nonzero coefficient in the second equation is that of $D^{k+2}$, and the resulting equation is:

$$
(k-1) m_{k-1} D_{x} a_{4}-4 a_{4} D_{x} m_{k-1}=0 .
$$

Multiplying by an integrating factor $a_{4}^{-(k-1) / 4-1}$ allows to write

$$
D_{x}\left[a_{4}^{-(k-1) / 4} m_{k-1}\right]=0 .
$$

This implies that

$$
m_{k-1}=\mu_{k-1} a_{4}^{(k-1) / 4}
$$

where $\mu_{k-1}$ is a constant.

- The next nonzero coefficient is that of $D^{k+1}$, implying that

$$
\begin{aligned}
& (k-2) m_{k-2} D_{x} a_{4}-4 a_{4} D_{x} m_{k-2}-3 a_{3} D_{x} m_{k-1}-6 a_{4} D_{x x} m_{k-1} \\
& +\frac{1}{2}(k-1) m_{k-1}\left[(k-1) D_{x x} a_{4}+2 D_{x} a_{3}-D_{x x} a_{4}\right]=0
\end{aligned}
$$

Substituting the value found for $m_{k-1}$ and using the integrating factor $a_{4}^{-(k-2) / 4-1}$ yields

$$
D_{x}\left[a_{4}^{-(k-2) / 4} m_{k-2}\right]=\mu_{k-1}\left[\frac{k-1}{4} D_{x}\left(\frac{a_{3}}{a_{4}^{3 / 4}}\right)+\frac{(k-1)(k-5)}{2} D_{x x}\left(a_{4}^{1 / 4}\right)\right] .
$$


Thus

$m_{k-2}=\mu_{k-2} a_{4}^{(k-2) / 4}+\mu_{k-1} a_{4}^{(k-2) / 4}\left[\frac{k-1}{4} \frac{a_{3}}{a_{4}^{3 / 4}}+\frac{(k-1)(k-5)}{8} \frac{D_{x} a_{4}}{a_{4}^{3 / 4}}\right]$,

being $\mu_{k-2}$ a constant. This constant appears because of the linearity of the equations, and introduces a redundancy in our solution $M$ ( $k$ is arbitrary). For illustrative reasons we keep it (and analogous constants) in the next few paragraphs.

- The highest nonzero coefficient in the first equation corresponds to $D^{k+3}$, yielding

$$
\begin{aligned}
k l_{k} D_{x} a_{4}-4 a_{4} D_{x} l_{k}+2 a_{4} D_{t} m_{k-1}+m_{k-1} D_{t} a_{4} & \\
& -a_{4} b_{1} D_{x} m_{k-1}+(k-1) a_{4} m_{k-1} D_{x} b_{1}=0 .
\end{aligned}
$$

Using again an integrating factor $a_{4}^{-(k-1) / 4-1}$ and the expression for $m_{k-1}$, it is found that

$$
D_{x}\left[a_{4}^{-k / 4} l_{k}\right]=\mu_{k-1}\left[\frac{k-1}{4} D_{x}\left(\frac{b_{1}}{a_{4}^{1 / 4}}\right)-\frac{k+1}{2} D_{t}\left(\frac{1}{a_{4}^{1 / 4}}\right)\right]
$$

A first integrability condition appears here: $\rho_{0}:=a_{4}^{-1 / 4}$ must be the density of a conservation law

$$
D_{t}\left(\frac{1}{a_{4}^{1 / 4}}\right)=D_{x} \sigma_{0} .
$$

This is an obstruction that narrows the class of equations 2.3.1 with $n=4, m=1$ that are integrable. Supposing that this condition is met, we set

$$
l_{k}=\lambda_{k} a_{4}^{k / 4}+\mu_{k-1}\left[\frac{k-1}{4} a_{4}^{(k-1) / 4} b_{1}-\frac{k+1}{2} a_{4}^{k / 4} \sigma_{0}\right] .
$$

- The coefficient of $D^{k+2}$, after substituting the definition of a second integrability condition

$$
D_{t}\left(\frac{a_{3}}{a_{4}}\right)=D_{x} \sigma_{1}
$$


implies that

$$
\begin{gathered}
D_{x}\left[a_{4}^{-(k-1) / 4} l_{k-1}\right]=\mu_{k-2}\left[\frac{k-2}{4} D_{x}\left(\frac{b_{1}}{a_{4}^{1 / 4}}\right)-\frac{k}{2} D_{x} \sigma_{0}\right] \\
+\lambda_{k}\left[\frac{1}{4} k D_{x}\left(\frac{a_{3}}{a_{4}^{3 / 4}}\right)+\frac{k(k-4)}{2} D_{x}^{2}\left(a_{4}^{1 / 4}\right)\right] \\
+\mu_{k-1}\left[\frac{\left(k^{2}-3 k-2\right)(k-1)}{4} D_{x}\left(a_{4}^{1 / 4} D_{x} \sigma_{0}\right)\right. \\
+\frac{(k-1)^{2}}{16} D_{x}\left(\frac{a_{3} b_{1}}{a_{4}}\right)+\frac{\left(k^{2}-5 k+5\right)(k-1)}{32} D_{x}\left(\frac{b_{1} D_{x} a_{4}}{a_{4}}\right) \\
-\frac{k(k+1)}{8} D_{x}\left(\frac{\sigma_{0} a_{3}}{a_{4}^{3 / 4}}\right)+\frac{(4-k) k(k+1)}{4} D_{x}^{2}\left(a_{4}^{1 / 4} \sigma_{0}\right)+\frac{k+1}{8} D_{x} \sigma_{1} \\
\left.+\frac{k-1}{4} D_{x} b_{0}+\frac{(k-1)(k-5)}{8} D_{x x} b_{1}\right]
\end{gathered}
$$

and we can find the expression of $l_{k-1}$ accordingly, in the form $l_{k-1}=\lambda_{k-1} a_{4}^{(k-1) / 4}+\lambda_{k}[\cdots]+\mu_{k-2}[\cdots]+\mu_{k-1}[\cdots]$.

- The coefficient in the second equation of $D^{k}$ leads to another conservation law

$$
D_{t}\left(\frac{b_{1}}{\sqrt{a_{4}}}-2 \frac{\sigma_{0}}{a_{4}^{1 / 4}}\right)=D_{x} \sigma_{2}
$$

and to an expression of $m_{k-3}$ of the form $m_{k-3}=\mu_{k-3} a_{4}^{(k-3) / 4}+$ $\lambda_{k}[\cdots]+\mu_{k-2}[\cdots]+\mu_{k-1}[\cdots]$.

- The computation continues producing an infinite system of equations that can be solved with a recursive scheme. In fact, in 6.1.4 the terms with highest order in $D$ come from $[L, A]$ and $[M, A]$ and produce equations where the unknowns $m_{i}, l_{j}$ appear in one term that can be isolated in the lhs of two equations of the form

$$
\begin{aligned}
& D_{x}\left(a_{4}^{-i / 4} l_{i}\right)=A_{i}, \quad i=k, k-1, \ldots, \\
& D_{x}\left(a_{4}^{-j / 4} m_{j}\right)=B_{j}, \quad j=k-1, k-2, \ldots
\end{aligned}
$$

The right hand sides $A_{i}, B_{j}$ of these equations must be total derivatives, so there appear an infinite number of integrability obstructions (that we will see are in the form of conservation laws) and arbitrary constants of integration in each of the terms of $L$ and $M$. We will justify a posteriori that these obstructions do not depend on $k$.

- In practice, one can implement the procedure taking $k=0$ and solving 2.3.27 in the order $j=0, j=-1, i=1, i=0$ and then alternating $j=-l, i=-l+1$ for $l \geq 2$. Of course, to completely 
solve the problem of integrability in this way one should perform an infinite number of steps. Usually, a small number of steps quickly reduces the possible subfamilies of equations 2.3.I admitting FRO's, and other procedures are used to prove full integrability of these remaining cases. For example, finding an explicit recursion operator (and seed or root symmetries) is enough to prove integrability.

In the following results we deal with the space of formal recursion operators of an equation that satisfies all the integrability conditions imposed by 2.3.27, and $\lambda_{i}$ and $\mu_{j}$ are the integration constants arising from its integration.

Proposition 16. A basis of the vector space the formal recursion operators of an integrable equation 2.3.I for $m=4$ is formed by the operators

$$
\begin{aligned}
\mathcal{L}_{i} & :=\left\{L+M D_{t}: \lambda_{i}=1 ; \lambda_{i^{\prime}}=0, i^{\prime} \neq i ; \mu_{j}=0\right\}, \quad \\
\mathcal{M}_{j} & :=\left\{L+M D_{t}: \lambda_{i}=0 ; \mu_{j}=1 ; \mu_{j^{\prime}}=0, j^{\prime} \neq j\right\}, \quad \text { Z } .
\end{aligned}
$$

The leading coefficients of $\mathcal{L}_{i}$ and $\mathcal{M}_{j}$ are

$$
\begin{aligned}
\mathcal{L}_{i}= & a_{4}^{i / 4} D^{i}+\left[\frac{i}{4} a_{3} a_{4}^{\frac{i-1}{4}-\frac{3}{4}}+\frac{(i-4) i}{2} a_{4}^{\frac{i-1}{4}} D_{x}\left(a_{4}^{1 / 4}\right)\right] D^{i-1}+\cdots \\
& +\left\{\left(\frac{i}{4} a_{4}^{\frac{i-4}{4}} b_{1}-\frac{i}{2} \sigma_{0} a_{4}^{\frac{i-3}{4}}\right) D^{i-3}+\cdots\right\} D_{t}, \\
\mathcal{M}_{j}= & {\left[\frac{j}{4} b_{1} a_{4}^{\frac{j}{4}}-\frac{j+2}{2} \sigma_{0} a_{4}^{\frac{j+1}{4}}\right] D^{j+1}+\cdots } \\
& +\left\{a_{4}^{\frac{j}{4}} D^{j}+\left[\frac{j}{4} a_{3} a_{4}^{\frac{j-4}{4}}+\frac{(j-4)) j}{2} D_{x}\left(a_{4}^{1 / 4}\right) a_{4}^{\frac{j-1}{4}}\right] D^{j-1}+\cdots\right\} D_{t}
\end{aligned}
$$

We have the following important technical results, whose proof can be found in Quintero and Heredero [39].

\section{Lemma 17.}

1. $\mathcal{L}_{i}=\mathcal{L}_{1}^{i}$ for all $i \in \mathbf{Z}$.

2. $\mathcal{M}_{-2}^{2}=\mathcal{L}_{0}=\mathcal{I}$.

3. $\mathcal{M}_{-2} \mathcal{L}_{1}=\mathcal{L}_{1} \mathcal{M}_{-2}=\mathcal{M}_{-1}$.

4. $\mathcal{M}_{j}=\mathcal{M}_{-2} \mathcal{L}_{j+2}$, $\mathcal{L}_{j}=\mathcal{M}_{-2} \mathcal{M}_{i-2}$. for all $i, j \in \mathbf{Z}$.

For ease of notation, in what follows we continue using the notation $s^{o}+s^{o-1}+\cdots$ to denote a formal series $\sum_{i=0}^{\infty} s^{o-i}$.

Lemma 18. Any pseudo-differential operator $\mathcal{O}$ of degree $(r, s)$

$$
\mathcal{O}=o_{r} D^{r}+o_{r-1} D^{r-1}+\cdots+\left[p_{s} D^{s}+p_{s-1} D^{s-1}+\cdots\right] D_{t}
$$


can be written as

$$
\mathcal{O}=p_{k} \mathcal{L}_{1}^{k}+p_{k-1} \mathcal{L}_{1}^{k-1}+\cdots+\left[q_{l} \mathcal{M}_{0} \mathcal{L}_{1}^{l}+q_{m-1} \mathcal{M}_{0} \mathcal{L}_{1}^{l-1}+\cdots\right] D_{t}
$$

with $p_{i}, q_{j}$ appropriate differential functions.

Theorem 19. Any formal recursion operator $\mathscr{R}=L+M D_{t}$ of an integrable equation 2.3.1 with $m=4$ can be expressed as

$$
\mathscr{R}=\alpha_{k} \mathcal{L}_{1}^{k}+\alpha_{k-1} \mathcal{L}_{1}^{k-1}+\cdots+\left[\beta_{l} \mathcal{M}_{0} \mathcal{L}_{1}^{l}+\beta_{l-1} \mathcal{M}_{0} \mathcal{L}_{1}^{l-1}+\cdots\right] D_{t}
$$

with $\alpha_{i}, \beta_{j}$ appropriate constants.

Theorem 20. Given a differential equation 2.3.I for $m=4$ the conditions of integrability, i.e. the conditions of existence of local formal recursion operators $\mathcal{L}_{1}, \mathcal{M}_{0}$, have the form of conservation laws

$$
D_{t} \rho_{i}=D_{x} \sigma_{i}
$$

Proof. Lemma 18 implies that, in particular, both $D_{x}$ and $D_{t}$ can be written as

$$
\begin{gathered}
D_{x}=\rho_{1} \mathcal{L}_{1}+\rho_{0}+\rho_{-1} \mathcal{L}_{1}^{-1}+\cdots+\pi_{-2} \mathcal{M}_{0} \mathcal{L}_{1}^{-2}+\pi_{-3} \mathcal{M}_{0} \mathcal{L}_{1}^{-3}+\cdots, \\
D_{t}=\sigma_{1} \mathcal{L}_{1}+\sigma_{0}+\sigma_{-1} \mathcal{L}_{1}^{-1}+\cdots+\theta_{0} \mathcal{M}_{0}+\theta_{-1} \mathcal{M}_{0} \mathcal{L}_{1}^{-1}+\cdots
\end{gathered}
$$

Commutativity of $D_{x}$ and $D_{t}$ implies the result.

We give in Appendix iii for further reference, a table of the first integrability conditions for equations in the class 2.3.1.

The homogeneity scheme can be used to study the symmetry structure of any integrable equation of the type 2.3.1. There exist two possible structures:

1. an algebra of FRO's generated by two independent operators, when $m$ is even

2. an algebra of FRO s generated by one operator, when $m$ is odd.

2.4 EXTENDED SYMMETRY APPROACH AND LAGRANGIAN SYSTEMS CLASSIFICATION.

The symmetry approach can treat Lagrangian systems if one applies it directly to the corresponding Euler-Lagrange equations. We are interested in second order Lagrangian systems with densities of the type

$$
\mathcal{L}=\frac{1}{2} L_{2}\left(u_{x x}, u_{x}, u\right) u_{t}^{2}+L_{1}\left(u_{x x}, u_{x}, u\right) u_{t}+L_{0}\left(u_{x x}, u_{x}, u\right)
$$

whose EL equations are of type $(4,1)$. They can be classified by the symmetry approach method once the explicit integrability conditions 
We will see in the following chapters

how

non-evolutionary equations arise from Lagrangian densities as per the vanishing of their variational derivative or

Euler-Lagrange operator. are determined in the form of conserved densities resulting from applying the extended symmetry approach to equations of the form 2.2.4 with $(n, m)=(4,1)$

$$
u_{t t}=H\left(u_{x x x x}, u_{x x x}, u_{x t}, u_{x x}, u_{t}, u_{x}, u\right)
$$

This is due to second order Lagrangian densities of the type 2.4.I having Euler-Lagrange equations

$$
\frac{\delta \mathcal{L}}{\delta u}=D_{x}^{2} \frac{\partial \mathcal{L}}{\partial u_{x x}}-D_{t} \frac{\partial \mathcal{L}}{\partial u_{t}}-D_{x} \frac{\partial \mathcal{L}}{\partial u_{x}}+\frac{\partial \mathcal{L}}{\partial u}=0
$$

resulting in equations of the form 2.4.2 with r.h.s $H$ not depending on $t t$-derivatives and whose integrability, as seen in 2.2 , can be determined by the extended formal symmetry approach as per the results reported in Heredero, Shabat, and Sokolov [16] and the extensions to fourth order equations in Quintero and Heredero [39].

This family of equations includes relevant systems like

$$
\begin{array}{lc}
\text { NLS-like } & \mathcal{L}=\frac{1}{2}\left(u_{t}+u_{x x}-u_{x}^{2}\right)^{2} \\
\text { Boussinesq } & \mathcal{L}=\frac{1}{2}\left(u_{t}+u_{x x}\right)^{2}+\frac{1}{2} u_{x}^{3} \\
\text { Landau-Lifshitz } & \mathcal{L}=\frac{\left(u_{t}+u_{x x}-\frac{1}{2} R^{\prime}(u)\right)^{2}}{4\left(u_{x}^{2}-R(u)\right)}+\frac{1}{12} R^{\prime \prime}(u)
\end{array}
$$

For more information about these and many others integrable systems, the reader can check Shabat et al. [43].

Example 21. For the NLS equation, derived from $\mathcal{L}=\frac{1}{2}\left(u_{t}+u_{x x}-u_{x}^{2}\right)^{2}$ as

$$
u_{t t}=u_{x x x x}+4 u_{x} u_{x t}+2 u_{x x} u_{t}-6 u_{x}^{2} u_{x x}
$$

explicit integrability conditions can be calculated and applied following the extended symmetry approach in order to find its generalized symmetries and class as an integrable case of 2.4.1.

The following theorem is adapted from publication [39].

Theorem 22. If a differential equation of the form

$$
u_{t t}=f\left(u, u_{x}, \ldots, u_{n}, u_{t}, u_{x t}, \ldots, u_{m t}\right)
$$

admits infinitely many symmetries of arbitrarily high order

$$
u_{\tau}=g\left(u, u_{x}, \ldots, u_{r}, u_{t}, u_{x t}, \ldots, u_{s t}\right)
$$

then it admits a formal recursion operator of the form

$$
\mathcal{R}=l_{1} D+l_{0}+l_{-1} D^{-1} \cdots+\left(1+m_{-1} D^{-1}+m_{-2} D^{-2}+\cdots\right) D_{t}
$$

where $l_{i}$ and $m_{j}$ are differential functions of the variables $u_{k}, u_{t l}$. 
This theorem can be used to solve the following two types of problems:

1. Classifying a family of equations of the form 2.4.4. Following the formal symmetry approach to the classification of integrable equations, the symmetry defining equations must be solved, with all operators $L, M, A$ and $B$ as unknowns. We will see how the extended symmetry approach is able to provide the solution to this problem.

2. Determining if a given equation 2.4 .4 is integrable. It could be achieved by finding an admitted FRO

$$
\mathcal{R}:=L+M D_{t}
$$

by solving the equations derived form the extended symmetry condition 2.3.9. The operators $L$ and $M$ are the unknowns of the equations, whereas $A$ and $B$ are data given by the r.h.s. $F$ of the given equation.

The finer structure of the space of matrix FROs strongly depends on the differential order of the r.h.s. of equations 2.4.4, i.e. on $n$ and $m$. The case of some third order equations $(n=3, m=1)$ was already discussed in section 2.2.

In this thesis we focus our considerations on type 1 problems restricted to the case of equations 2.4.2 with $n=4, m=1$ that produce a more complicated structure in the space of solutions of the symmetry condition 2.2.6. Problems of type 2 are sketched in chapter 6.

Example 23. Following section 2.2, the explicit integrability conditions have been written in the form of conserved densities and recently published in [39]. A few of the corresponding canonical densities are listed in table 2.1. 


$$
\begin{aligned}
\rho_{0}= & a_{4}^{-1 / 4} \\
\rho_{1}= & a_{4}^{-1 / 2} b_{1}-2 a_{4}^{-1 / 4} \sigma_{0} \\
\rho_{2}= & \frac{a_{3}}{a_{4}} \\
\rho_{3}= & 12 \frac{a_{3}^{2}}{a_{4}^{7 / 4}}-4 \frac{b_{1}^{2}}{a_{4}^{3 / 4}}-32 \frac{a_{2}}{a_{4}^{3 / 4}}-16 \frac{\sigma_{0}^{2}}{a_{4}^{3 / 4}}-16 \frac{\sigma_{1}}{a_{4}^{1 / 4}}-12 \frac{a_{3}\left(D_{x} a_{4}\right)}{a_{4}^{7 / 4}} \\
& +5 \frac{\left(D_{x} a_{4}\right)^{2}}{a_{4}^{7 / 4}} \\
\rho_{4}= & 2 \frac{b_{1} a_{3}}{a_{4}^{5 / 4}}-4 \frac{\sigma_{2}}{a_{4}^{1 / 4}}-8 \frac{b_{0}}{a_{4}^{1 / 4}}-3 \frac{b_{1}\left(D_{x} a_{4}\right)}{a_{4}^{5 / 4}} \\
\rho_{5}= & \sigma_{0} \rho_{3}-\sigma_{3} \rho_{0}
\end{aligned}
$$

where $a_{i}=\frac{\partial H}{\partial u_{i}}$ and $b_{i}=\frac{\partial H}{\partial u_{t i}}$.

Table 2.1: Table of conserved densities of equations of the form $u_{t t}=$ $H\left(u_{x x x x}, u_{x x x}, u_{x t}, u_{x x}, u_{t}, u_{x}, u\right)$

Thus, with the above characterization of the integrability of equations 2.4.2 we can apply our symmetry-based definition of integrability to the classification of integrable Lagrangian systems. This is done with the method presented in chapter 1 and the required adaptations to the extended formal symmetry approach. The detailed calculations of this process, together with its results in the form of integrable Lagrangian system classes representatives are given in chapter 5 . It relies on the integrability conditions given in table 2.1, which are further detailed in the appendix. 
As seen so far in preceding chapters:

- Coordinate transformations provide equivalences in the set of all equations permitting the definition of classes in it. The application of contact transformations (as defined in this chapter) preserves the integrability of differential equations as they are changes of coordinates on $J^{(n)}$. As a consequence we are allowed to use them in order to work with simpler equivalent expressions provided that they preserve the characteristics of the class we are working on. We will refer to such transformations as allowed transformations.

- Integrability is closely related to linearization. We already discussed a change of variables (Cole-Hopf transformation) relating a non-linear equation with a linear one which is integrable as it possesses a recursion operator.

In this chapter we give the basis of transformations in jet spaces from the computational point of view. For this we could consider generic differential substitutions that generically group all possible transformations between two commuting flows

$$
\begin{aligned}
& u_{t}=q\left(t, x, u, u_{1}, u_{2}, \cdots, u_{n}\right) \\
& u_{\tau}=s\left(t, x, u, u_{1}, u_{2}, \cdots, u_{m}\right)
\end{aligned}
$$

of an evolutionary equation and its evolutionary generalized symmetries as denoted in chapter 1 . However differential substitutions do not have an inverse in all cases, making them not suitable for strict classification in equivalence classes. For that reason, we will employ for that purpose only contact transformations.

\subsection{POINT TRANSFORMATIONS}

Point transformations do not depend on derivatives

$$
\begin{aligned}
t \rightarrow \bar{t} & =\chi(x, t, u) \\
x \rightarrow \bar{x} & =\xi(x, t, u) \\
u \rightarrow \bar{u} & =\psi(x, t, u)
\end{aligned}
$$


and those preserving the class of evolution equations, i.e. point allowed transformations for generic evolution equations, have the form

$$
\begin{aligned}
t \rightarrow \bar{t} & =\chi(t) \\
x \rightarrow \bar{x} & =\xi(x, u) \\
u \rightarrow \bar{u} & =\psi(x, u)
\end{aligned}
$$

Example 24. Equation

$$
u_{t}=u_{x x}+u_{x}^{2}+a e^{-u}+b
$$

becomes linear through differential substitution $u=\ln v$ producing

$$
v_{t}=v_{x x}+b v+a
$$

\subsection{CONTACT TRANSFORMATIONS AND CONTACT CONDITION}

For functions $u=u(x, t)$, transformations depending on $u$-derivatives $u_{J}$, up to $u_{x}$

$$
\begin{aligned}
t \rightarrow \bar{t}= & \chi\left(x, t, u, u_{x}\right) \neq 0 \\
x \rightarrow \bar{x}= & \xi\left(x, t, u, u_{x}\right) \\
u \rightarrow \bar{u}= & \psi\left(x, t, u, u_{x}\right) \\
& \text { with }\left|\chi_{u_{x}}\right|+\left|\xi_{u_{x}}\right|+\left|\psi_{u_{x}}\right| \neq 0
\end{aligned}
$$

are contact transformations if the transformed ("new") variables $\bar{u}_{\bar{x}}, \bar{u}_{\bar{t}}$ don't depend on $u_{x x}, u_{x t}, u_{t t}$.

These contact conditions follow from $\bar{u}_{x}=\bar{u}_{\bar{x}} \bar{x}_{x}, \bar{u}_{t}=\bar{u}_{\bar{t}} \bar{t}_{t}$ which yield

$$
\bar{u}_{\bar{x}}=\frac{\bar{u}_{x}}{\bar{x}_{x}}=\frac{D_{x}(\psi)}{D_{x}(\xi)}=\frac{\psi_{x}+\psi_{u} u_{x}+\psi_{u_{x}} u_{x x}}{\xi_{x}+\xi_{u} u_{x}+\xi_{u_{x}} u_{x x}}=H\left(x, t, u, u_{x}, u_{x x}\right)
$$

and by imposing that the transformed variables remain in the set of first order transformations we get as the contact conditions

$$
\begin{aligned}
H_{u_{x x}} & =0 \\
\psi_{u_{x}}\left(\xi_{x}+\xi_{u} u_{x}+\xi_{u_{x}} u_{x x}\right)-\left(\psi_{x}+\psi_{u} u_{x}+\psi_{u_{x}} u_{x x}\right) \xi_{u_{x}} & =0 \\
\psi_{u_{x}}\left(\xi_{x}+\xi_{u} u_{x}\right) & =\left(\psi_{x}+\psi_{u} u_{x}\right) \xi_{u_{x}}
\end{aligned}
$$

and

$$
\begin{aligned}
& \bar{u}_{\bar{t}}=\frac{\bar{u}_{t}}{\bar{t}_{t}}=\frac{D_{t}(\psi)}{D_{t}(\chi)}=\frac{\psi_{t}+\psi_{u} u_{t}+\psi_{u_{t}} u_{t t}}{\chi_{t}+\chi_{u} u_{t}+\chi_{u_{t}} u_{t t}}=G\left(x, t, u, u_{x}, u_{x x}, u_{t t}\right) \\
& G_{u_{t t}}=0 \\
& \psi_{u_{t}}\left(\chi_{t}+\chi_{u} u_{t}+\chi_{u_{t}} u_{t t}\right)-\left(\psi_{t}+\psi_{u} u_{t}+\psi_{u_{t}} u_{t t}\right) \chi_{u_{t}}=0 \\
& \psi_{u_{t}}\left(\chi_{t}+\chi_{u} u_{t}\right)=\left(\psi_{t}+\psi_{u} u_{t}\right) \chi_{u_{t}}
\end{aligned}
$$


The inverse transformation

$$
\begin{aligned}
\bar{t} \rightarrow t & =\chi^{-1}\left(\bar{x}, \bar{t}, \bar{u}, \bar{u}_{\bar{x}}\right) \\
\bar{x} \rightarrow x & =\xi^{-1}\left(\bar{x}, \bar{t}, \bar{u}, \bar{u}_{\bar{x}}\right) \\
\bar{u} \rightarrow u & =\psi^{-1}\left(\bar{x}, \bar{t}, \bar{u}, \bar{u}_{\bar{x}}\right)
\end{aligned}
$$

can be obtained by solving system (3.2.1) with $\bar{u}_{\bar{x}}=\frac{\psi_{\bar{u}_{\bar{x}}}}{\bar{\zeta}_{\overline{u_{\bar{x}}}}} \neq$ constant and $u_{\bar{x}}=u_{x} x_{\bar{x}}, u_{\bar{t}}=u_{t} t_{\bar{t}}$ as

$$
\begin{aligned}
& u_{x}=\frac{u_{\bar{x}}}{x_{\bar{x}}}=\frac{D_{\bar{x}}\left(\psi^{-1}\right)}{D_{\bar{x}}\left(\zeta^{-1}\right)}=\frac{\psi_{\bar{x}}^{-1}+\psi_{\bar{u}}^{-1} \bar{u}_{\bar{x}}+\psi_{\bar{u}_{\bar{x}}}^{-1} \bar{u}_{\overline{x x}}}{\bar{\zeta}_{\bar{x}}^{-1}+\xi_{\bar{u}}^{-1} \bar{u}_{\bar{x}}+\bar{\zeta}_{\bar{u}_{\bar{x}}}^{-1} \bar{u}_{\overline{x x}}} \\
& u_{t}=\frac{u_{\bar{t}}}{t_{\bar{t}}}=\frac{D_{\bar{t}}\left(\psi^{-1}\right)}{D_{\bar{t}}\left(\chi^{-1}\right)}=\frac{\psi_{\bar{t}}^{-1}+\psi_{\bar{u}}^{-1} \bar{u}_{\bar{t}}+\psi_{\bar{u}_{\bar{t}}}^{-1} \bar{u}_{\overline{t t}}}{\chi_{\bar{t}}^{-1}+\chi_{\bar{u}}^{-1} \bar{u}_{\bar{t}}+\chi_{\bar{u}_{\bar{t}}}^{-1} \bar{u}_{\overline{t t}}}
\end{aligned}
$$

Again, allowed contact transformations for evolution equations are only those with $\chi\left(x, t, u, u_{x}\right)=\chi(t)$.

\subsection{METHOD FOR THE CONSTRUCTION OF CONTACT TRANSFOR-} MATIONS

From the specification of the function $\xi\left(x, t, u, u_{x}\right)$ in (3.2.1), the relations (3.2.2), (3.2.2) can be viewed as a linear partial differential equation for the second function $\psi\left(x, t, u, u_{x}\right)$. The corresponding characteristic system of ordinary differential equations

$$
\frac{d x}{1}=\frac{d u}{u}=-\frac{\xi_{u} d u}{\xi_{x}+u \xi_{u}}
$$

admits the first integral $\xi\left(x, t, u, u_{x}\right)=$ constant. Then it follows that in order to obtain $\psi=\psi\left(x, t, u, u_{x}\right)$ we need to solve the ODE above.

Example 25. Legrende transformations for $u=u(x)$

$$
\begin{gathered}
\bar{x}=u_{x} \\
\bar{u}=x u_{x}-u \\
\bar{u}_{\bar{x}}=\frac{\bar{u}_{x}}{\bar{x}_{x}}=\frac{D_{x}(\psi)}{D_{x}(\tilde{\xi})}=\frac{\psi_{x}+\psi_{u} u_{x}+\psi_{u_{x}} u_{x x}}{\xi_{x}+\bar{\zeta}_{u} u_{x}+\bar{\zeta}_{u_{x}} u_{x x}}=\frac{u_{x}-u_{x}+x u_{x x}}{u_{x x}}=x \\
x=\bar{u}_{\bar{x}} \\
u=\bar{x} \bar{u}_{\bar{x}}-\bar{u} \\
u_{x}=\frac{u_{\bar{x}}}{x_{\bar{x}}}=\frac{D_{\bar{x}}\left(\psi^{-1}\right)}{D_{\bar{x}}\left(\bar{\zeta}^{-1}\right)}=\frac{\psi_{\bar{x}}^{-1}+\psi_{\bar{u}}^{-1} \bar{u}_{\bar{x}}+\psi_{\bar{u}_{\bar{x}}}^{-1} \bar{u}_{\overline{x x}}}{\xi_{\bar{x}}^{-1}+\bar{\zeta}_{\bar{u}}^{-1} \bar{u}_{\bar{x}}+\bar{\zeta}_{\bar{u}_{\bar{x}}}^{-1} \bar{u}_{\overline{x x}}}=\frac{\bar{u}_{\bar{x}}-\bar{u}_{\bar{x}}+\bar{x} \bar{u}_{\overline{x x}}}{\bar{u}_{\overline{x x}}}=\bar{x}
\end{gathered}
$$




\subsection{CONTACT TRANSFORMATIONS FOR EVOLUTION AND NON EVO- LUTIONARY EQUATIONS}

Summarizing the calculations above, generically a change of variables

$$
\begin{aligned}
& \bar{u}=\psi\left(x, u, u_{x}\right) \\
& \bar{x}=\phi\left(x, u, u_{x}\right)
\end{aligned}
$$

for equations in this class, has contact condition

$$
\psi_{u_{x}}\left(\phi_{u} u_{x}+\phi_{x}\right)=\phi_{u_{x}}\left(\psi_{u} u_{x}+\psi_{x}\right)
$$

and more generically (see Sokolov [46]) contact fields allow changes of coordinates in the algebra $\mathscr{A}$ of differential functions

$$
g\left(\bar{x}, \bar{u}, \frac{D(\psi)}{D(\phi)}\right)=\left(\psi_{\bar{u}_{\bar{x}}}-\frac{D(\psi)}{D(\phi)} \xi_{\bar{u}_{\bar{x}}}\right) g\left(x, u, u_{x}\right)
$$

A contact generic transformation (3.2.1) for an evolution equation $u_{t}=F[u]$ into a new evolution equation $\bar{u}_{\bar{t}}=\bar{F}[\bar{u}]$ not depending on $\bar{t}$ :

$$
\bar{F}_{\bar{t}}=\bar{u}_{\overline{t t}}=G_{\bar{t}}=G_{\chi}=0
$$

must have the form

$$
\begin{aligned}
t \rightarrow \bar{t} & =\chi t \\
x \rightarrow \bar{x} & =\xi\left(x, u, u_{x}\right), \quad \xi_{u_{x}} \neq 0 \\
u \rightarrow \bar{u} & =\psi\left(x, u, u_{x}\right), \quad \psi_{u_{x}} \neq 0
\end{aligned}
$$

being the contact conditions

$$
\begin{aligned}
\psi_{u_{x}}\left(\xi_{x}+\xi_{u} u_{x}\right) & =\left(\psi_{x}+\psi_{u} u_{x}\right) \xi_{u_{x}} \\
\psi_{u_{t}} & =0
\end{aligned}
$$

Again, using the notation and results in Sokolov [46] as depicted at the end of the preceding chapter, we can state the following properties

1. The commutator of the flows of a given evolutionary equation $u_{t}=q[u]$ and its classical or contact symmetries $s_{\tau}=s[u]$

$$
\begin{aligned}
{[q, s] } & =s^{*}(q)-q^{*}(s) \\
& =\left(\partial_{1} s \partial_{0} q-\partial_{1} q \partial_{0} q\right) u_{1} \\
& +\partial_{1} s \partial_{x} q-\partial_{1} q \partial s \\
& +q \partial_{0} s-s \partial_{0} q
\end{aligned}
$$

does not depend on $u_{2}$, this is, contact symmetries form a Lie sub-algebra of generalized symmetries. 
2. This sub-algebra $\mathfrak{S}$ is finite-dimensional, with $\operatorname{dim}_{\mathbf{C}} \mathfrak{S} \leqslant n+3$ for a given $n$-th order equation $u_{t}=q[u]$.

Example 26. Third order evolution equations

$$
\bar{u}_{t}=\bar{u}_{\bar{x} \bar{x} \bar{x}}+\bar{u}_{\bar{x}}^{3}
$$

"new" and "old" variables as defined above are reversed in this example

Transform to

$$
\begin{aligned}
\bar{u}_{\bar{x}} & =\frac{1}{D \phi} D \psi=\frac{\psi_{u_{x}} u_{x x}+\psi_{u} u_{x}+\psi_{x}}{\phi_{u_{x}} u_{x x}+\phi_{u} u_{x}+\phi_{x}}=\frac{\psi_{u} u_{x}+\psi_{x}}{\phi_{u} u_{x}+\phi_{x}} \\
u_{x} & =-\frac{\psi_{x}-\phi_{x} \bar{u}_{\bar{x}}}{\psi_{u}-\phi_{u} \bar{u}_{\bar{x}}} \\
\bar{u}_{\bar{x} \bar{x}} & =\frac{1}{D \phi} D \frac{\psi_{u} u_{x}+\psi_{x}}{\phi_{u} u_{x}+\phi_{x}}=\frac{A u_{x x}+B}{\left(\phi_{u} u_{x}+\phi_{x}\right)^{2} D \phi} \\
\bar{u}_{\bar{x} \bar{x} \bar{x}} & =\left(\frac{1}{D \phi} D\right)^{2} \frac{\psi_{u} u_{x}+\psi_{x}}{\phi_{u} u_{x}+\phi_{x}}=\frac{J}{\left(\phi_{u} u_{x}+\phi_{x}\right)(D \phi)^{3}} u_{x x x}+\cdots \\
\bar{u}_{\bar{t}} & =\left(D_{t}-\frac{D_{t} \phi}{D \phi} D\right) \psi
\end{aligned}
$$

with $J=\psi_{u} \phi_{x}-\psi_{x} \phi_{u}$. For derivatives in the new time variable we have

$$
\begin{aligned}
\bar{u}_{\bar{t}} & =\left(D_{t}-\frac{D_{t} \phi}{D \phi} D\right) \psi=\psi_{u_{x}} u_{x t}+\psi_{u} u_{t}-\frac{\phi_{u_{x}} u_{x t}+\phi_{u} u_{t}}{\phi_{u_{x}} u_{x x}+\phi_{u} u_{x}+\phi_{x}}\left(\psi_{u_{x}} u_{x x}+\psi_{u} u_{x}+\psi_{x}\right) \\
& =\psi_{u_{x}} u_{x t}+\psi_{u} u_{t}-\frac{\phi_{u_{x}} u_{x t}+\phi_{u} u_{t}}{\phi_{u} u_{x}+\phi_{x}}\left(\psi_{u} u_{x}+\psi_{x}\right) \\
& =\left(\psi_{u_{x}}-\frac{\psi_{u} u_{x}+\psi_{x}}{\phi_{u} u_{x}+\phi_{x}} \phi_{u_{x}}\right) u_{x t}+\left(\psi_{u}-\frac{\psi_{u} u_{x}+\psi_{x}}{\phi_{u} u_{x}+\phi_{x}} \phi_{u}\right) u_{t} \\
& =\left(\psi_{u}-\frac{\psi_{u} u_{x}+\psi_{x}}{\phi_{u} u_{x}+\phi_{x}} \phi_{u}\right) u_{t}=\left[\frac{\psi_{u}\left(\phi_{u} u_{x}+\phi_{x}\right)-\left(\psi_{u} u_{x}+\psi_{x}\right) \phi_{u}}{\phi_{u} u_{x}+\phi_{x}}\right] u_{t} \\
& =\frac{J}{\phi_{u} u_{x}+\phi_{x}} u_{t}
\end{aligned}
$$

From where

$$
\begin{aligned}
u_{t} & =\frac{\phi_{u} u_{x}+\phi_{x}}{J} \bar{u}_{\bar{t}}=\frac{\phi_{u} u_{x}+\phi_{x}}{J}\left(\bar{u}_{\bar{x} \bar{x} \bar{x}}+\bar{u}_{\bar{x}}^{3}\right) \\
& =\frac{\phi_{u} u_{x}+\phi_{x}}{J}\left[\frac{J}{\left(\phi_{u} u_{x}+\phi_{x}\right)(D \phi)^{3}} u_{x x x}+\cdots+\left(\frac{\psi_{u} u_{x}+\psi_{x}}{\phi_{u} u_{x}+\phi_{x}}\right)^{3}\right] \\
& =\frac{1}{(D \phi)^{3}} u_{x x x}+\cdots+\frac{1}{J} \frac{\left(\psi_{u} u_{x}+\psi_{x}\right)^{3}}{\left(\phi_{u} u_{x}+\phi_{x}\right)^{2}}
\end{aligned}
$$

Meaning that an allowed contact transformation for evolution equations introduces a non constant separant defined as the coefficient of the highest order derivative.

In this example, as detailed in Mikhailov, Shabat, and Sokolov [26], the original equation is related to the modified Korteweg-de-Vries 
equation by a the differential substitution $v=u_{x}$ (introduction of a potential)

$$
\begin{gathered}
v_{t}=v_{x x x}+3 v^{2} v_{x} \\
u_{x t}=u_{x x x x}+3 u_{x}^{2} u_{x x}=D\left(u_{x x x}+u_{x}^{3}\right) \\
\Rightarrow u_{t}=u_{x x x}+u_{x}^{3}
\end{gathered}
$$

\subsection{CHANGE OF COORDINATES IN THE LAGRANGIAN SYSTEMS} CLASSIFICATION

Here we derive the allowed form of a generic contact transformation

$$
\begin{aligned}
\bar{t} & =\tau\left(u_{x}, u, x\right) \quad \bar{x}=\xi\left(u_{x}, u, x\right) \quad \bar{u}=\psi\left(u_{x}, u, x\right) \\
\psi_{u_{x}} D_{x} \xi & =\xi_{u_{x}} D_{x} \psi
\end{aligned}
$$

to try to simplify Lagrangian densities expressions appearing during the classification process introduced in chapter 2 . Lagrangian densities change according to the law

$$
\operatorname{det} J \cdot \overline{\mathcal{L}}+\operatorname{Div} P=\mathcal{L}, \quad J=\left(\begin{array}{cc}
D_{x} \xi & D_{t} \xi \\
D_{x} \tau & D_{t} \tau
\end{array}\right)
$$

so, first of all, $L_{2}$ changes according to the law

$$
\begin{aligned}
\tau^{\prime} D \xi \bar{L}_{2}\left(\bar{u}_{\bar{x} \bar{x}}, \bar{u}_{\bar{x}}, \bar{u}\right) \bar{u}_{\bar{t}}^{2} & =\frac{j^{2} D \xi}{\tau^{\prime}\left(\xi_{u} u_{x}+\xi_{x}\right)^{2}} \bar{L}_{0} u_{t}^{2}+O\left(u_{t}\right) \\
& =L_{2}\left(u_{x x}, u_{x}, u\right) u_{t}^{2}+O\left(u_{t}\right) . \\
& \Rightarrow L_{0}=\frac{j^{2}}{\tau^{\prime}\left(\xi_{u} u_{x}+\xi_{x}\right)} \bar{L}_{0}, \quad j=\psi_{u} \xi_{x}-\xi_{u} \psi_{x}
\end{aligned}
$$

Specifying to a linear $L_{2}=L_{01} u_{x x}+L_{00}$

$$
\begin{gathered}
\frac{\tau^{\prime}(\tilde{D} \xi)^{2}}{j^{2} D \xi}\left[L_{01}\left(\frac{j}{(\tilde{D} \xi)^{2}} \frac{u_{x x}}{D \xi}+\frac{\tilde{D}^{2} \psi \tilde{D} \xi-\tilde{D} \psi \tilde{D}^{2} \xi}{(\tilde{D} \xi)^{3}}\right)+L_{00}\right]= \\
\frac{\tau^{\prime}}{j^{2} D \xi}\left[\frac{j L_{01} u_{x x}}{D \xi}+\frac{\tilde{D}^{2} \psi \tilde{D} \xi-\tilde{D} \psi \tilde{D}^{2} \xi}{\tilde{D} \xi} L_{01}+(\tilde{D} \xi)^{2} L_{00}\right]
\end{gathered}
$$

Thus, a linear dependence of $L_{2}$ on $u_{x x}$ is contact-removable.

We will make use of all of these facts in the classification detailed in Part II. 
In this chapter we describe the algorithms implemented for the calculations involved in the second order integrable Lagrangian systems classification presented as the main result of this thesis. This work has been divided into modules and algorithm templates for computations with the calculus techniques introduced in preceding chapters, focusing on those required for implementation of the classification of order $(2,4)$ integrable (as per the extended symmetry approach) evolutionary equations arising as Euler-Lagrange equations of second order Lagrangian systems, described in chapters 2 and 3 .

Calculations in jet spaces with arbitrary functions and higher order derivatives makes the help of a symbolic algebra computer system mandatory. In general all calculations are long size and time consuming and require extensive programming time, debugging and check of the results. The Mathematica computer algebra system was checked to implement the required symbolic computations and has been used to perform and verify all the calculations, which were organized in modules for

- base calculations like derivatives, prolongations, linearizations, product operations for differential and pseudo-differential operators,

- higher order variables definitions, auxiliary functions for total derivatives, jet variables functions, tables for formatting of expressions, integrators and solvers,

- algorithm templates for

- classifications of integrable equations by the integration of Canonical Conservation Law (CCL)s,

- proofs of integrability,

- calculation and integration of determining equations of point and contact symmetries,

- coordinate changes, contact and point transformations,

- calculation of recursion operators.

These modules are briefly described in this chapter.

\subsection{SYMBOLIC VECTOR CALCULUS FUNCTIONS}

The fundamental calculus functions were organized in a collection of symbolic calculus routines written by this Ph.D. thesis director, Dr. 
Rafael Hernández Heredero. This vector calculus module features functions for expression formatting, partial and total derivatives, variational derivatives, prolonged vector fields, Lie brackets and commutators, and generic higher order differential and pseudo-differential algebra.

Listing 4.I shows how basic functions are employed to verify calculations for a conservation law of the modified KdV equation.

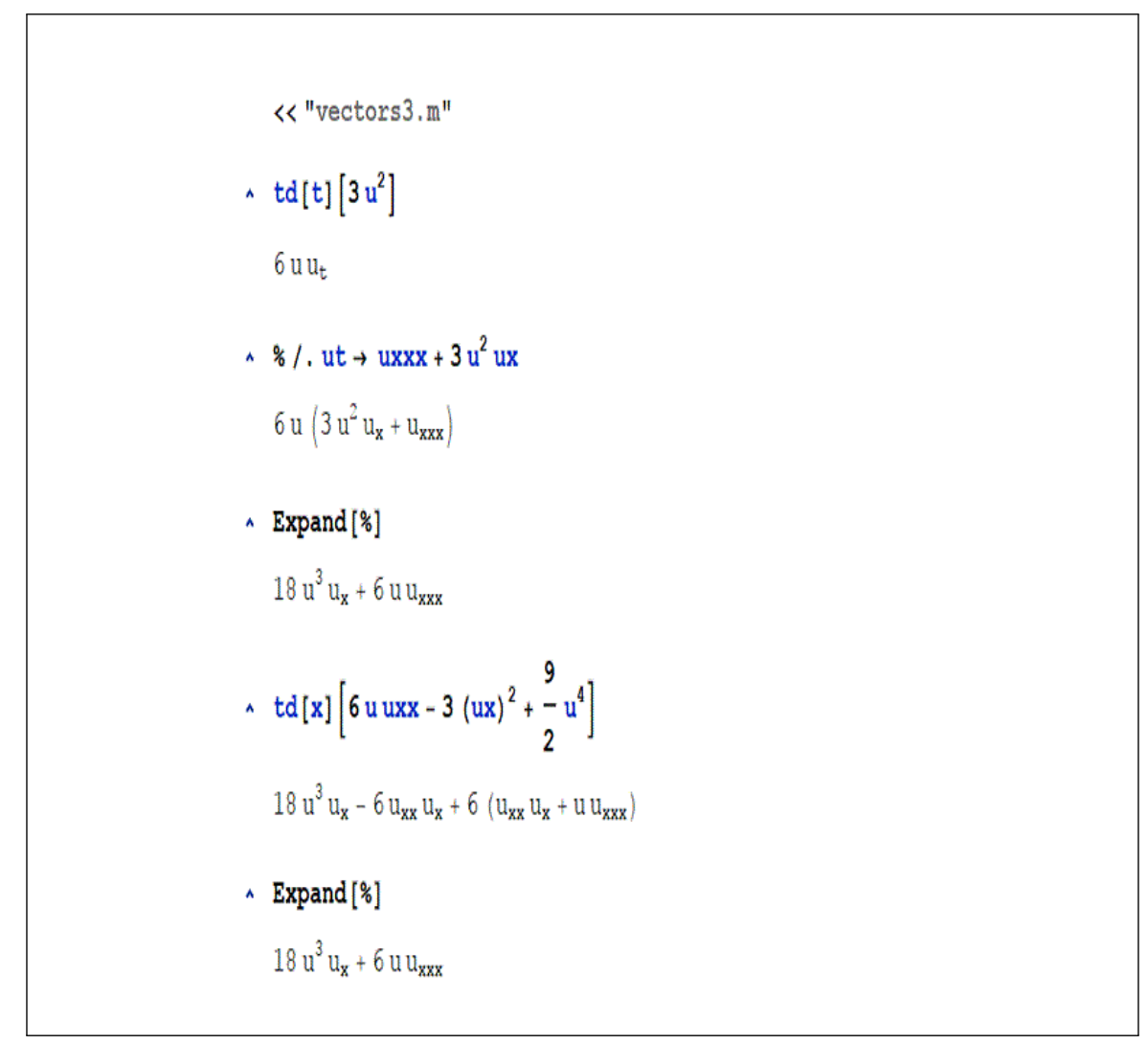

Listing 4.1: Total derivatives functions and verification of conservation law for $\mathrm{mKdV}$.

\subsection{INTEgRATION TECHNiQue}

The method employed in our calculations is based on the one depicted in figure 4.2.1. In this flow chart we summarized the fundamental technique of second order evolution equations classification. The integrability conditions are given data and also, on contrary to the case of evolutionary equations, the outputs of the procedure are known as per the integrable equations listings of integrable families or cases, described in chapter 1 and available in the literature. 


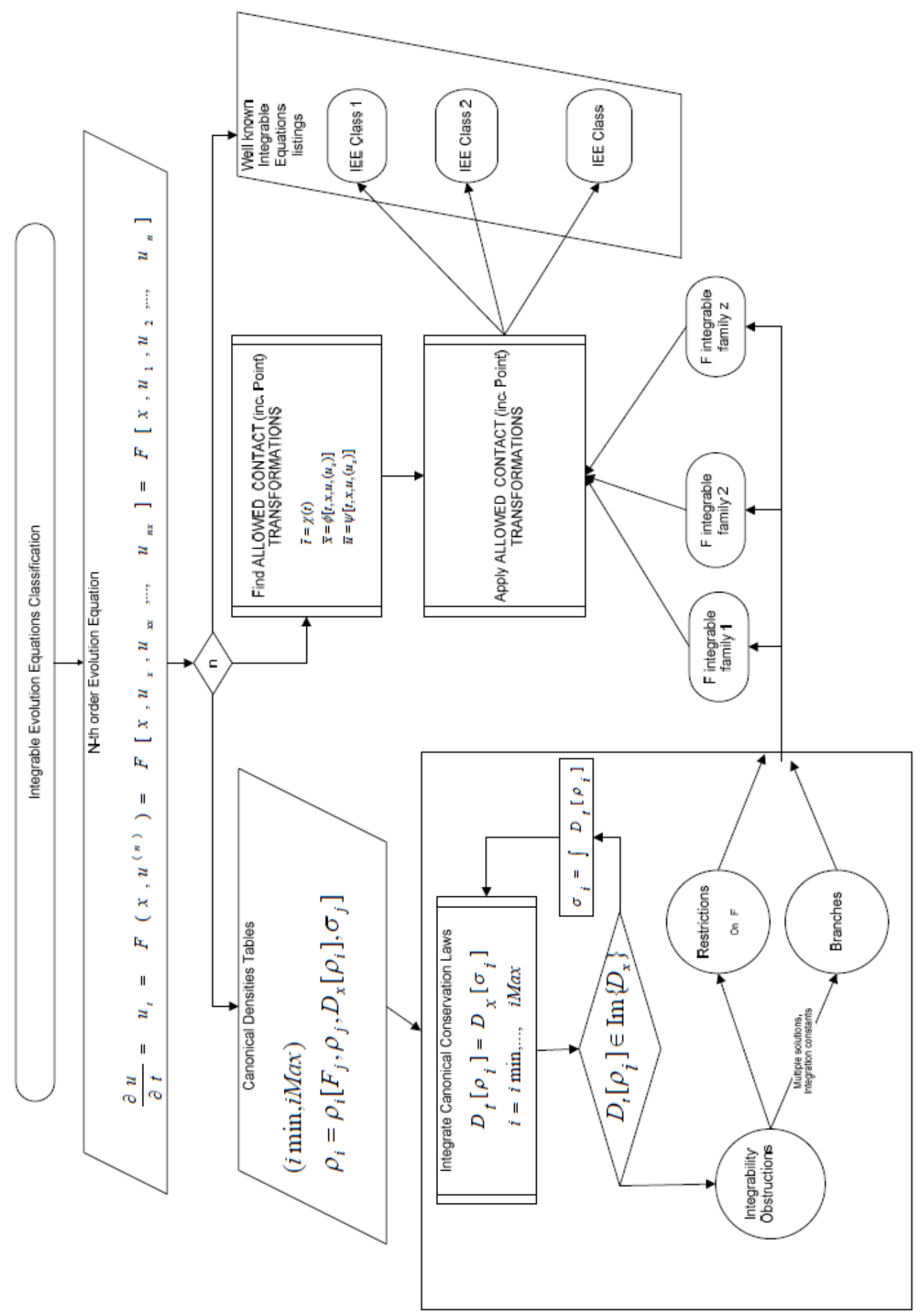

Figure 4.2.1: Computations flow for IEEC

The adapted procedure for ILSC is depicted in figure 4.2.2 and consists of 
Working from the top down (step 3) has proved to be the most feasible method with these calculations.
1. Sequentially ${ }^{1}$ compute canonical conservation laws

$$
d_{i}:=D_{t}\left(\rho_{i}\right)-D_{x}\left(\sigma_{i}\right)=0, \quad i=0,1,2, \ldots
$$

bounded to $i=i_{M a x}$ as the number of previously computed canonical densities $\rho_{i}$, and by defining

$$
\sigma_{i}:=\sigma_{i}\left(\sigma \operatorname{Vars}\left[\left(\rho_{i}\right)_{t}\right]\right)
$$

this is setting the jet variables on which $\sigma_{i}$ depend as the correspondent integrated variables to those existing in $\left(\rho_{i}\right)_{t}$.

2. Analysis of the expanded CCL. There exist different approaches to the problem of implementing differential algebra calculations. In order to work with expanded canonical conserved densities and conservation laws, due to their long expressions

- it is recommended to analyze their computation process (see figure 4.2.2) in detail and make use of disk storage for intermediate expressions.

- it is not advisable to implement computations in a "bruteforce" fashion but to pinpoint which information is required and find a suitable quick method to in order to extract it from the correspondent expanded expression.

3. Find highest order integrability obstruction. These were defined in section 1.11 and computed as the coefficients in $d_{i}$ (step 1 ) of the highest order jet variable w.r.t. which $d_{i}$ is a polynomial expression. Recursively taking partial derivatives results in integrability obstructions.

4. Integrate integrability obstruction. This is solving the differential equation obstruction $=0$.

5. Setting solutions found in step 4 as restrictions to the form of the Lagrangian density $\mathcal{L}$. This in turn gives new expressions for the Euler-Lagrange equations and canonical densities.

6. Go to step 1 , until all obstructions (bounded to $i=i_{\text {Max }}$ ) are integrated.

1 Although theoretically this calculation can be done simultaneously (i.e: using vector variables), that method strongly constraints feasibility and efficiency. 


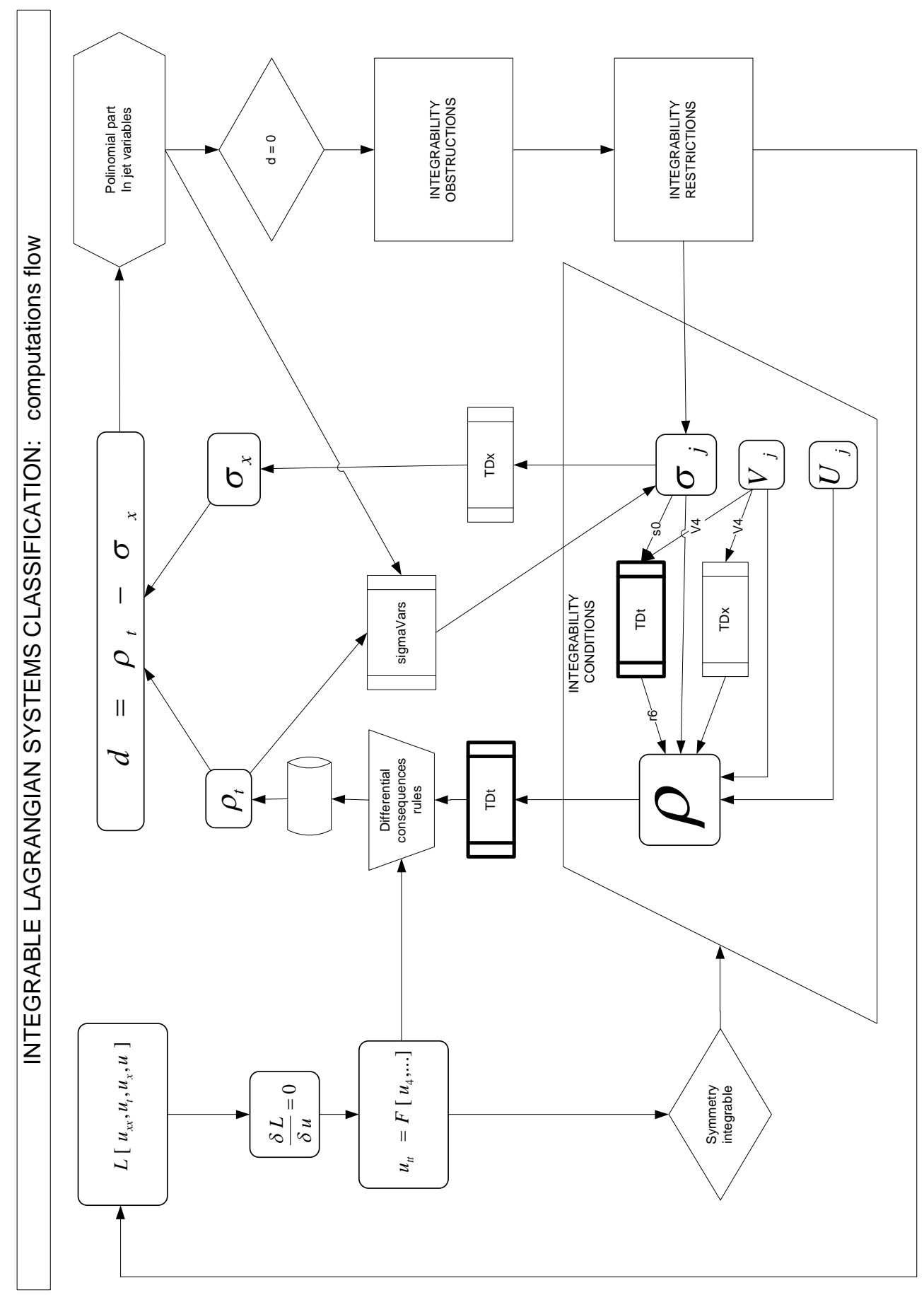

Figure 4.2.2: Computations flow for ILSC 


\subsection{OBSTRUCTIONS ANALYSIS}

Some key features of the algorithms above are listed as sample code and screenshot.

Obstructions extraction previous to integration

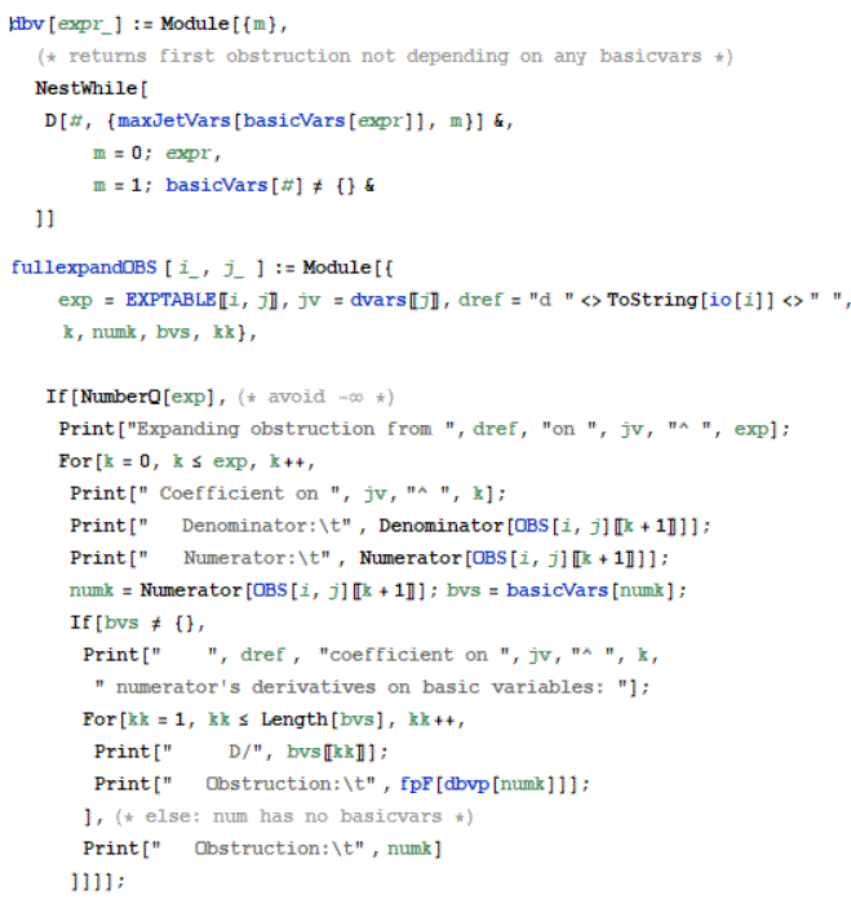

Listing 4.2: Sample of CCL Integration template code 


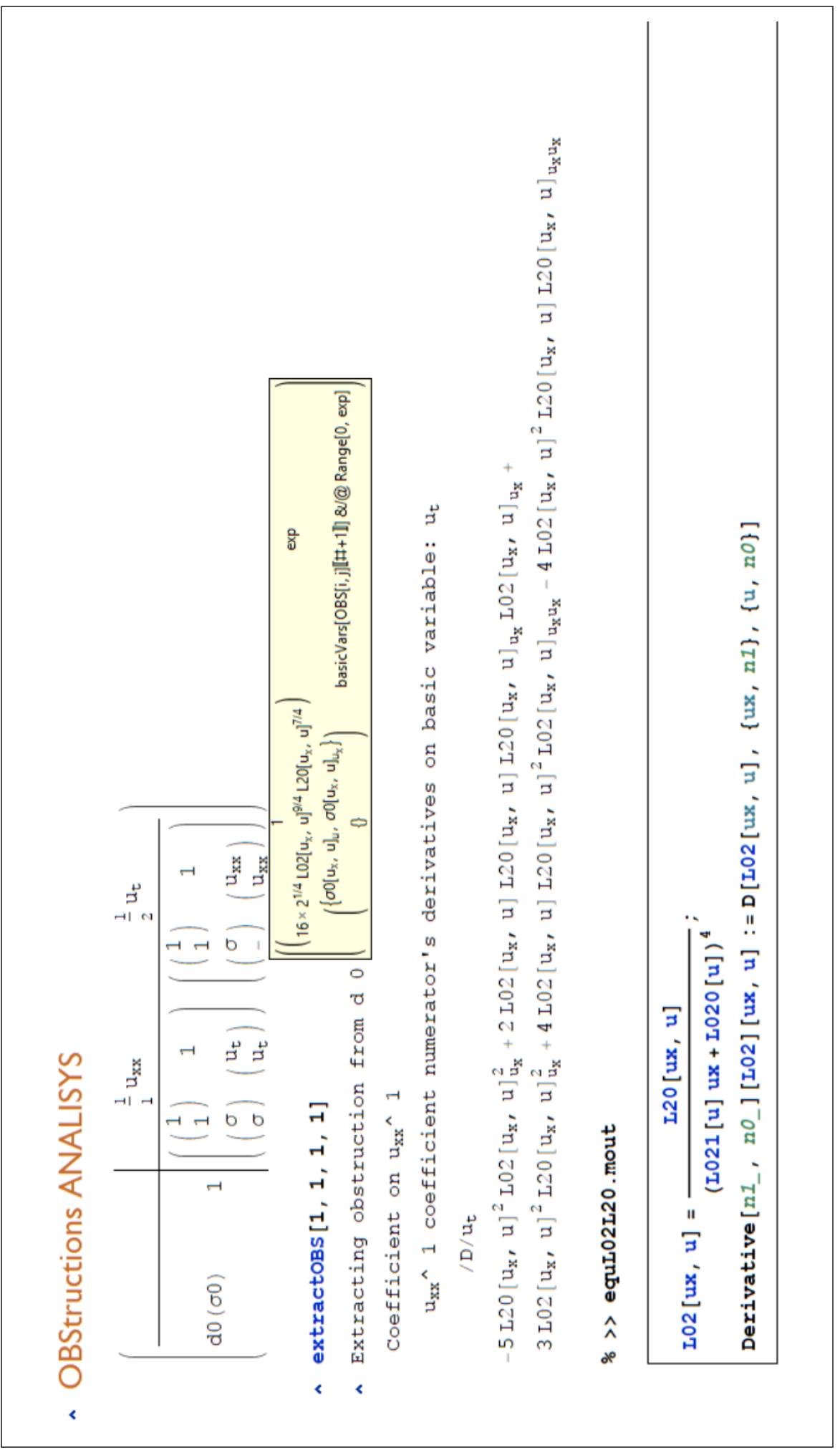

Listing 4.3: Sample screenshot 
With these computations all the cases were checked to be integrable, however the

C-integrability mentioned in the introduction can only be proved by finding ( $a$ construction method

for) recursion operators for each of the final cases, which is the aim of chapter 6.

\subsection{PROOFS OF INTEGRABILITY}

As mentioned in section 4.2 the classification technique has the canonical conserved densities tables $\left\{\rho_{i}\right\}$ as an input, in this section we are going to generate their correspondent fluxes $\left\{\sigma_{i}\right\}$ from the integrable cases computed in the ILSC.

In order to verify integrability ${ }^{2}$ of the final cases, we are reversing the CCL integration procedure thus generating conservation laws. The number or these being theoretically infinite as we can generate one from each canonical density which are infinitely many (though calculations are bounded to $i_{M a x}=7$ ) due to they have been generated through formal recursion operators.

Listing 4.4 shows a sample of the algorithm template for the first Lagrangian density case found $\mathcal{L}_{1}$, and fourth CCL resulting in the final check

$$
d_{3}=\left(\rho_{3}\right)_{t}-\left(\sigma_{3}\right)_{x}=0
$$

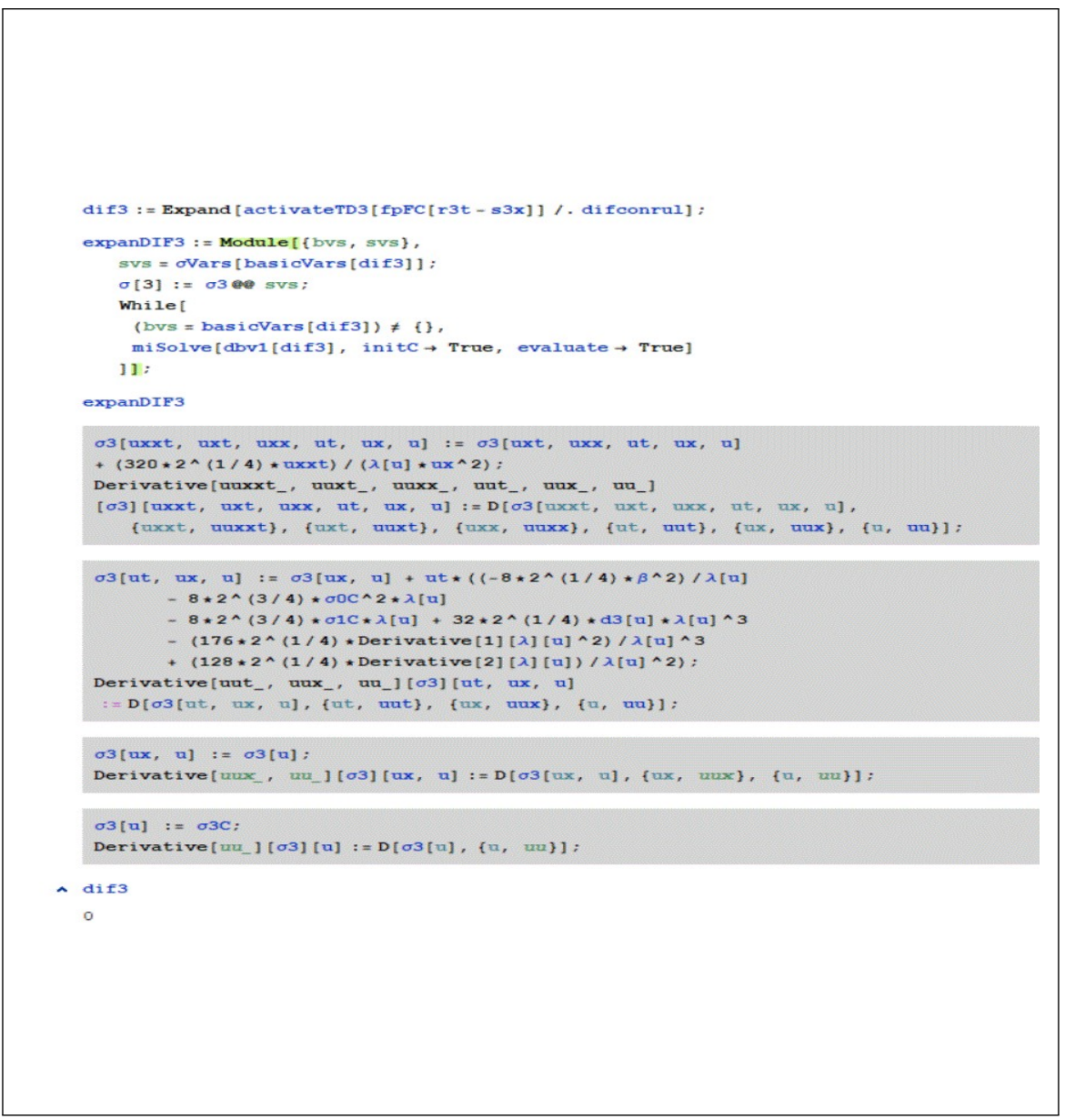

Listing 4.4: Sample of code template for proof of integrability

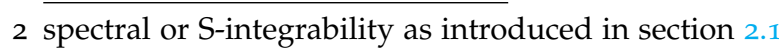


Part II

RESULTS AND OPEN PROBLEMS 

SECOND ORDER INTEGRABLE LAGRANGIAN SYSTEMS CLASSIFICATION

\subsection{INITIAL CONDITIONS}

For second order Lagrangian systems with Lagrangian density

$$
\mathcal{L}=\frac{1}{2} L_{2}\left(u_{x x}, u_{x}, u\right) u_{t}^{2}+L_{1}\left(u_{x x}, u_{x}, u\right) u_{t}+L_{0}\left(u_{x x}, u_{x}, u\right)
$$

initial conditions are

$$
L_{2}\left(u_{x x}, u_{x}, u\right) \neq 0
$$

and

$$
\frac{\partial^{2} L_{2}\left(u_{x x}, u_{x}, u\right)}{\partial u_{x x}^{2}}, \frac{\partial^{2} L_{1}\left(u_{x x}, u_{x}, u\right)}{\partial u_{x x}^{2}}, \frac{\partial^{2} L_{0}\left(u_{x x}, u_{x}, u\right)}{\partial u_{x x}^{2}}
$$

not allowed to vanish simultaneously.

These conditions are derived from the Euler-Lagrange equations of (5.1.1) (as per 1.9.1 and 2.4.3):

$$
\begin{aligned}
\frac{\delta \mathcal{L}}{\delta u} & =-D_{t} \frac{\partial \mathcal{L}}{\partial u_{t}}+D_{x}^{2} \frac{\partial \mathcal{L}}{\partial u_{x x}}-D_{x} \frac{\partial \mathcal{L}}{\partial u_{x}}+\mathcal{L}_{u} \\
& =-D_{t} \mathcal{L}_{u_{t}}+D_{x}^{2} \mathcal{L}_{u_{x x}}-D_{x} \mathcal{L}_{u_{x}}+\mathcal{L}_{u}=0
\end{aligned}
$$

yielding for 5.1.1:

$$
\begin{aligned}
u_{t t} & =\frac{1}{L_{2}} \frac{\partial^{2} \mathcal{L}}{\partial u_{x x}^{2}} u_{x x x x}+\frac{1}{L_{2}} \frac{\partial^{3} \mathcal{L}}{\partial u_{x x}^{3}} u_{x x x}^{2}+\frac{1}{L_{2}} \frac{\partial L_{2}}{\partial u_{x x}} u_{x t}^{2} \\
& +\frac{2}{L_{2}}\left(\frac{\partial^{3} \mathcal{L}}{\partial u_{x x}^{2} \partial u_{t}} u_{x t}+\frac{\partial^{3} \mathcal{L}}{\partial u_{x x}^{2} \partial u_{x}} u_{x x}+\frac{\partial^{3} \mathcal{L}}{\partial u_{x x}^{2} \partial u} u_{x}\right) u_{x x x} \\
& +\frac{2}{L_{2}}\left[\left(\frac{\partial^{2} L_{2}}{\partial u_{x x} \partial u_{x}} u_{x x}+\frac{\partial^{2} L_{2}}{\partial u_{x x} \partial u} u_{x}-\frac{\partial L_{2}}{\partial u_{x}}\right) u_{t}\right. \\
& \left.+\frac{\partial^{2} L_{1}}{\partial u_{x x} \partial u_{x}} u_{x x}+\frac{\partial^{2} L_{1}}{\partial u_{x x} \partial u} u_{x}-\frac{\partial L_{1}}{\partial u_{x}}\right] u_{x t} \\
& +\frac{1}{2 L_{2}}\left(\frac{\partial^{3} L_{2}}{\partial u_{x x} \partial^{2} u_{x}} u_{x x}^{2}+\cdots\right) u_{t}^{2} \\
& +\frac{1}{L_{2}}\left(\frac{\partial^{3} L_{1}}{\partial u_{x x} \partial u_{x}^{2}} u_{x x}^{2}+2 \frac{\partial^{3} L_{0}}{\partial u_{x x} \partial u_{x} \partial u} u_{x} u_{x x}+\frac{\partial^{2} L_{1}}{\partial u_{x x} \partial u} u_{x x}-\frac{\partial^{2} L_{0}}{\partial u_{x}^{2}} u_{x x}\right. \\
& +\frac{\partial^{3} L_{1}}{\partial u_{x x} \partial u^{2}} u_{x}^{2}-\frac{\partial^{2} L_{0}}{\partial u_{x} \partial u} u_{x} u_{t}+\cdots
\end{aligned}
$$


which can more briefly be written

$$
\begin{aligned}
& u_{t t}=\frac{1}{L_{2}} \mathcal{L}_{u_{x x} u_{x x}} u_{x x x x}+\frac{1}{L_{2}} \mathcal{L}_{u_{x x} u_{x x} u_{x x}} u_{x x x}^{2}+\frac{1}{L_{2}} L_{2} u_{x x} u_{x t}^{2} \\
& +\frac{2}{L_{2}}\left(\mathcal{L}_{u_{x x}^{2} u_{t}} u_{x t}+\mathcal{L}_{u_{x x}^{2} u_{x}} u_{x x}+\mathcal{L}_{u_{x x}^{2} u} u_{x}\right) u_{x x x} \\
& +\frac{2}{L_{2}}\left[\left(L_{2} u_{x x} u_{x} u_{x x}+L_{2 u_{x x} u} u_{x}-L_{2 u_{x}}\right) u_{t}\right. \\
& \left.+L_{1 u_{x x} u_{x}} u_{x x}+L_{1 u_{x x} u} u_{x}-L_{1 u_{x}}\right] u_{x t} \\
& +\frac{1}{2 L_{2}}\left(L_{2} u_{x x} u_{x} u_{x} u_{x x}^{2}+\cdots\right) u_{t}^{2} \\
& +\frac{1}{L_{2}}\left(L_{1} u_{x x} u_{x} u_{x} u_{x x}^{2}+2 L_{0 u_{x x} u_{x} u} u_{x} u_{x x}+L_{1 u_{x x} u} u_{x x}-L_{0 u_{x} u_{x}} u_{x x}\right. \\
& \left.+L_{1 u_{x x} u u} u_{x}^{2}-L_{0 u u} u_{x}\right) u_{t}+\cdots
\end{aligned}
$$

this is of the generic form

$$
u_{t t}=F\left(u_{x x x x}, u_{x x x}, u_{x t}, u_{x x}, u_{t}, u_{x}, u\right)
$$

being integrable in the sense described in the extended symmetry approach Heredero, Shabat, and Sokolov [16], and with extensions to fourth order equations as published in [39]. Having a nonzero or nonsingular equation's "separant" (see Heredero [15]) of 5.1.8 defined by

$$
\left(\frac{\partial F}{\partial u_{x x x x}}\right)^{-1 / 4}
$$

that amounts in our case to the nonvanishing of

$$
\frac{1}{L_{2}}\left(\frac{1}{2} \frac{\partial^{2} L_{2}}{\partial u_{x x}^{2}} u_{t}^{2}+\frac{\partial^{2} L_{1}}{\partial u_{x x}^{2}} u_{t}+\frac{\partial^{2} L_{0}}{\partial u_{x x}^{2}}\right)=F_{u_{x x x x}}
$$

imposes neither $L_{2}=0$ nor $L_{2}, L_{1}$ and $L_{0}$ being simultaneously linear in $u_{x x}$.

\subsection{INTEGRABILITY CONDITIONS FROM CANONICAL CONSERVA- TION LAWS}

The first canonical conservation law (cf. the Appendix)

$$
D_{t}\left(\rho_{0}\right)=D_{x}\left(\sigma_{0}\right)
$$

produces the first obstruction to integrability

$$
\left(3 L_{2} u_{x x} u_{x x} L_{2} u_{x x}+L_{2} L_{2} u_{x x} u_{x x} u_{x x}\right) L_{2} u_{x x} u_{x x}=0
$$

from the coefficient of $u_{x x x}^{2} u_{t}^{3}$ in $d_{0}=\left(\rho_{0}\right)_{t}-\left(\sigma_{0}\right)_{x}=0$ with

$$
\left(\rho_{0}\right)_{t}=D_{t}\left[\left(\frac{\partial F}{\partial u_{x x x x}}\right)^{-1 / 4}\right]=D_{x} \sigma_{0}
$$


The solutions to differential equation 5.2.2 are

$$
\begin{aligned}
L_{2 G}\left(u_{x x}, u_{x}, u\right) & =\sqrt{L_{22}\left(u_{x}, u\right) u_{x x}^{2}+L_{21}\left(u_{x}, u\right) u_{x x}+L_{20}\left(u_{x}, u\right)} \\
L_{2 S}\left(u_{x x}, u_{x}, u\right) & =L_{21}\left(u_{x}, u\right) u_{x x}+L_{20}\left(u_{x}, u\right) .
\end{aligned}
$$

Solution $L_{2 G}$ shows up to be incompatible with further integrability conditions from the first canonical conservation law. So setting $L_{2}:=$ $L_{2 S}$ in 5.1.1 and recalculating $d_{0}$ we can find the next integrability obstruction coming from term $u_{x t}^{2} u_{t}$ in $d_{0}$

$$
L_{21}\left(u_{x}, u\right)\left(-3 \frac{\partial^{2} L_{1}}{\partial u_{x x}^{2}}-\frac{\partial^{3} L_{1}}{\partial u_{x x}^{3}} \frac{\partial^{2} L_{1}}{\partial u_{x x}^{2}} u_{x x}\right)-L_{20}\left(u_{x}, u\right) \frac{\partial^{3} L_{1}}{\partial u_{x x}^{3}} \frac{\partial^{2} L_{1}}{\partial u_{x x}^{2}}=0
$$

Further calculations with $d_{0}=D_{t}\left(\rho_{0}\right)-D_{x}\left(\sigma_{0}\right)$ result in new integrability obstructions whose solution $\frac{\partial^{2} L_{0}}{\partial u_{x x}^{2}}$ is not compatible with the initial condition 5.1.3.

At this point we must look at equation 5.1.8 in order find a contact transformation allowing the integration of 5.2.4. Setting $L_{21}\left(u_{x}, u\right)=$ 0 we have $L_{2}\left(u_{x x}, u_{x}, u\right)=L_{20}\left(u_{x}, u\right)$ and we find the next integrability condition to be

$$
\frac{\partial^{2} L_{1}}{\partial u_{x x}^{2}}=0
$$

derived from obstruction in coefficient of $d_{0}$ in $u_{x x x}^{2} u_{t}$

$$
\frac{\partial^{3} L_{1}}{\partial u_{x x}^{3}} \frac{\partial^{2} L_{1}}{\partial u_{x x}^{2}}=0
$$

so we set

$$
L_{1}\left(u_{x x}, u_{x}, u\right):=L_{11}\left(u_{x}, u\right) u_{x x}+L_{10}\left(u_{x}, u\right)
$$

which in turn can be simplified to

$$
L_{1}\left(u_{x x}, u_{x}, u\right):=L_{10}\left(u_{x}, u\right)
$$

by using Lagrangian equivalence.

After re-computing $d_{0}$ we find that the former condition on $u_{x x x} u_{x t}$ has changed after this setting to

$$
L_{20}\left(u_{x}, u\right)^{1 / 4}\left(5\left(\frac{\partial^{3} L_{0}}{\partial u_{x x}^{3}}\right)^{2}-4 \frac{\partial^{4} L_{0}}{\partial u_{x x}^{4}} \frac{\partial^{2} L_{0}}{\partial u_{x x}^{2}}\right)=0
$$

with $L_{20}\left(u_{x}, u\right) \neq 0$. This equation is solved by setting

$$
L_{0}\left(u_{x x}, u_{x}, u\right):=L_{02}\left(u_{x}, u\right) u_{x x}^{2}+L_{01}\left(u_{x}, u\right) u_{x x}+L_{00}\left(u_{x}, u\right)
$$

with $L_{01}\left(u_{x}, u\right):=0$ again as per Lagrangian equivalence, leaving

$$
L_{0}\left(u_{x x}, u_{x}, u\right):=L_{02}\left(u_{x}, u\right) u_{x x}^{2}+L_{00}\left(u_{x}, u\right)
$$


and

$$
\mathcal{L}=\frac{1}{2} L_{20}\left(u_{x}, u\right) u_{t}^{2}+L_{10}\left(u_{x}, u\right) u_{t}+L_{02}\left(u_{x}, u\right) u_{x x}^{2}+L_{00}\left(u_{x}, u\right)
$$

as the form of the initial Lagrangian density 5.1.1 where we have removed the initial dependence of the arbitrary functions

$$
L_{2}\left(u_{x x}, u_{x}, u\right), L_{1}\left(u_{x x}, u_{x}, u\right), L_{0}\left(u_{x x}, u_{x}, u\right)
$$

on $u_{x x}$.

After recomputing the first canonical conservation law and setting variables in $\sigma_{0}$, the condition on $u_{x x} u_{t}$ yields a differential equation relating $L_{20}\left(u_{x}, u\right)$ and $L_{02}\left(u_{x}, u\right)$

$$
\begin{aligned}
5 L_{20}^{2}\left(\frac{\partial L_{02}}{\partial u_{x}}\right)^{2}-2 L_{20} L_{02} \frac{\partial L_{02}}{\partial u_{x}} \frac{\partial L_{20}}{\partial u_{x}} & + \\
3 L_{02}^{2}\left(\frac{\partial L_{20}}{\partial u_{x}}\right)^{2} & +4 L_{02} L_{20}^{2} \frac{\partial^{2} L_{02}}{\partial u_{x}^{2}}+ \\
& +4 L_{02}^{2} L_{20} \frac{\partial^{2} L_{20}}{\partial u_{x}^{2}}=0
\end{aligned}
$$

which can be put in operator form as

$$
\begin{aligned}
\left(\frac{\partial}{\partial u_{x}}+\frac{1}{4} L_{20}^{-1} \frac{\partial L_{20}}{\partial u_{x}}\right)^{2} z & =0 \\
z & :=L_{02}^{-1 / 4}
\end{aligned}
$$

and with general solution

$$
L_{02}\left(u_{x}, u\right):=\frac{L_{20}\left(u_{x}, u\right)}{\left(L_{021}(u) u_{x}+L_{020}(u)\right)^{4}}
$$

which once set generates integrability condition $\frac{\partial L_{020}}{\partial u}=0$ imposing

$$
L_{020}(u):=\text { constant }:=K_{020}
$$

After computing $\left(\rho_{1}\right)_{t}$ with the constrains to $u_{t t}=F$ obtained from the first canonical conservation law, and setting the correspondent jet variables in $\sigma_{1}$, one finds the following differential equation as obstruction to integrability, from the coefficient of $u_{x x}^{3}$ in $d_{1}=\left(\rho_{1}\right)_{t}-$ $\left(\sigma_{1}\right)_{x}$

$$
\begin{array}{r}
-6 L_{021}(u)+L_{20}\left(u_{x}, u\right)\left(\frac{\partial L_{20}}{\partial u_{x}}\right)^{2}+6 K_{020}\left(\frac{\partial L_{20}}{\partial u_{x}}\right)^{3}+ \\
6 L_{021}(u)\left(\frac{\partial L_{20}}{\partial u_{x}}\right)^{3} u_{x}+3 L_{021}(u) L_{20}^{2}\left(u_{x}, u\right) \frac{\partial L_{20}^{2}}{\partial u_{x}^{2}}+ \\
-6 K_{020} L_{20}\left(u_{x}, u\right) \frac{\partial^{2} L_{20}}{\partial u_{x}^{2}} \frac{\partial L_{20}}{\partial u_{x}}+ \\
-6 K_{020} L_{021}(u) L_{20}\left(u_{x}, u\right) \frac{\partial^{2} L_{02}}{\partial u_{x}^{2}} \frac{\partial L_{20}}{\partial u_{x}} u_{x}+ \\
+K_{020} L_{020}(u)^{2} \frac{\partial^{3} L_{20}}{\partial u_{x x}^{3}}+L_{021}(u) L_{020}(u)^{2} \frac{\partial^{3} L_{20}}{\partial u_{x x}^{3}} u_{x}=0
\end{array}
$$


Working like in the case of the first conservation law one can find that

$$
L_{20}\left(u_{x}, u\right):=\frac{L_{021}(u) u_{x}+K_{020}}{L_{202}(u) u_{x}^{2}+L_{201}(u) u_{x}+L_{200}(u)}
$$

is a solution to 5.2.16 that makes the generic expression of our Lagrangian look like

$$
\begin{aligned}
\mathcal{L} & =\frac{1}{2} \frac{L_{021}(u) u_{x}+K_{020}}{L_{202}(u) u_{x}^{2}+L_{201}(u) u_{x}+L_{200}(u)} u_{t}^{2} \\
& +L_{10}\left(u_{x}, u\right) u_{t} \\
& +\frac{u_{x x}^{2}}{\left(L_{021}(u) u_{x}+K_{020}\right)^{3}\left(L_{202}(u) u_{x}^{2}+L_{201}(u) u_{x}+L_{200}(u)\right)} \\
& +L_{00}\left(u_{x}, u\right)
\end{aligned}
$$

and yielding new obstructions to integrability among which we find an interesting relation for $L_{10}\left(u_{x}, u\right)$ in the coefficient of $d_{1}$ on $u_{x x} u_{t}$ :

$$
\begin{aligned}
\left(L_{202} u_{x}^{2}+L_{201} u_{x}+L_{200}\right) \frac{\partial^{3} L_{10}}{\partial u_{x}^{3}} & + \\
3\left(2 L_{202} u_{x}+L_{201}\right) \frac{\partial^{2} L_{10}}{\partial u_{x}^{2}}+6 L_{202} \frac{\partial L_{10}}{\partial u_{x}} & = \\
\frac{\partial^{3}}{\partial u_{x}^{3}}\left[\left(L_{202} u_{x}^{2}+L_{201} u_{x}+L_{200}\right) L_{10}\right] & =0
\end{aligned}
$$

which we will solve by setting

$$
L_{10}\left(u_{x}, u\right):=\frac{\frac{1}{2} A(u) u_{x}^{2}+B(u) u_{x}+C(u)}{L_{202}(u) u_{x}^{2}+L_{201}(u) u_{x}+L_{200}(u)} .
$$

while new obstructions for the form of $L_{00}\left(u_{x}, u\right)$ will be derived from canonical conservation laws $d_{i}=\left(\varrho_{i}\right)_{t}-\left(\sigma_{i}\right)_{x}, i \geq 1$.

From the coefficient of $d_{1}$ in $u_{x x}^{2}$ we find a clear dependence of the integrability conditions on the factors

$$
\begin{array}{r}
K_{020}, \\
L_{021}(u), \\
{\left[L_{202}(u) u_{x}^{2}+L_{201}(u) u_{x}+L_{200}(u)\right] .}
\end{array}
$$

At this point we start looking for ways to simplify $\mathcal{L}$ and to pinpoint which of the arbitrary functions in it will cause non-equivalent Lagrangian expressions or integrable cases. Taking into account that the undergoing classification is made with no dependence of the arbitrary functions $L_{2}\left(u_{x}, u\right), L_{1}\left(u_{x}, u\right), L_{0}\left(u_{x}, u\right)$ on $x$, we must see now which of the arbitrary functions are determining the branches for integrable cases.

Solution 5.2.17 suggest using point transformations

$$
\begin{aligned}
t & \rightarrow \chi(t) \\
x & \rightarrow \xi(x, t, u) \\
u & \rightarrow \psi(x, t, u)
\end{aligned}
$$


allowing normalization on $K_{020}, L_{021}(u)$ and permitting the leading coefficients 5.2.17 and 5.2.14 to be expressed in two different ways and with different expressions for arbitrary functions $L_{202}(u), L_{201}(u)$ and $L_{200}(u)$ in each case depending on the point transformations used.

- Case A

A suitable point transformation with $K_{020}=0, L_{021}(u) \neq 0$ transforms the coefficients on $u_{t}^{2}$ and $u_{x x}^{2}$ to

$$
\begin{aligned}
L_{20 A}\left(u_{x}, u\right) & :=\frac{L_{021}(u) u_{x}}{L_{202}(u) u_{x}^{2}+L_{201}(u) u_{x}+L_{200}(u)} \\
& =\frac{u_{x}}{\frac{L_{202}(u)}{L_{021}(u)} u_{x}^{2}+\frac{L_{201}(u)}{L_{021}(u)} u_{x}+\frac{L_{200}(u)}{L_{021}(u)}} \\
& :=\frac{u_{x}}{L_{202 A}(u) u_{x}^{2}+L_{201 A}(u) u_{x}+L_{200 A}(u)} \\
L_{02 A}\left(u_{x}, u\right) & :=\frac{u_{x x}^{2}}{L_{021}(u)^{4} u_{x}^{3}\left[L_{202 A}(u) u_{x}^{2}+L_{201 A}(u) u_{x}+L_{200 A}(u)\right]}
\end{aligned}
$$

- Case B

For $K_{020} \neq 0, L_{021}(u)=0$ we will have

$$
\begin{aligned}
L_{20 B}\left(u_{x}, u\right) & :=\frac{K_{020}}{L_{202}(u) u_{x}^{2}+L_{201}(u) u_{x}+L_{200}(u)} \\
L_{02 B}\left(u_{x}, u\right) & :=\frac{1}{K_{020}^{3}\left[L_{202}(u) u_{x}^{2}+L_{201}(u) u_{x}+L_{200}(u)\right]}
\end{aligned}
$$

$5 \cdot 3$ CASE A: $K_{020}=0, L_{021}(u) \neq 0$.

In this section we continue our analysis of integrability obstructions and correspondent setting of new expressions for arbitrary functions derived from the canonical conservation laws after setting

$$
\begin{aligned}
K_{020} & :=1 \\
L_{021}(u) & :=0 \\
L_{20}\left(u_{x}, u\right) & :=L_{20 A}\left(u_{x}, u\right)
\end{aligned}
$$

as allowed by the point transformation mentioned above, which makes the new equivalent Lagrangian

$$
\begin{aligned}
\mathcal{L}_{A} & :=\frac{1}{2} \frac{u_{x}}{L_{202 A}(u) u_{x}^{2}+L_{201 A}(u) u_{x}+L_{200 A}(u)} u_{t}^{2} \\
& +L_{10 A}\left(u_{x}, u\right) u_{t} \\
& +\frac{u_{x x}^{2}}{L_{021}(u)^{4} u_{x}^{3}\left(L_{202 A}(u) u_{x}^{2}+L_{201 A}(u) u_{x}+L_{200 A}(u)\right)} \\
& +L_{00 A}\left(u_{x}, u\right)
\end{aligned}
$$


with $L_{10 A}\left(u_{x}, u\right)$ as determined in 5.2.20 and adapted for case A

$$
L_{10 A}\left(u_{x}, u\right):=\frac{\frac{1}{2} A(u) u_{x}^{2}+B(u) u_{x}+C(u)}{L_{202 A}(u) u_{x}^{2}+L_{201 A}(u) u_{x}+L_{200 A}(u)}
$$

which will be set after the correspondent obstruction to integrability is found for each particular sub-case. We can open two sub-cases depending on $L_{200 A}(u)$ vanishing or not.

5.3.1 Case A-1: $L_{200 A}(u)=0$.

By setting $L_{200 A}(u):=0$ Lagrangian 5.3.I becomes

$$
\begin{aligned}
\mathcal{L}_{A} & :=\frac{1}{2} \frac{1}{L_{202 A}(u) u_{x}+L_{201 A}(u)} u_{t}^{2} \\
& +L_{10 A}\left(u_{x}, u\right) u_{t} \\
& +\frac{u_{x x}^{2}}{L_{021}(u)^{4} u_{x}^{3}\left(L_{202 A}(u) u_{x}^{2}+L_{201 A}(u) u_{x}\right)} \\
& +L_{00 A}\left(u_{x}, u\right)
\end{aligned}
$$

Permitted point transformations are

$$
\begin{aligned}
& t \rightarrow \chi(t) \\
& x \rightarrow \xi(x, t, u) \\
& u \rightarrow \psi(x, t, u)
\end{aligned}
$$

transforming $L_{2}$ into

$$
\bar{L}_{2}=\frac{\chi^{\prime}}{\psi_{u} \xi_{x}\left[\xi_{x} L_{202 A}(u)-\xi_{u} L_{201 A}(u)\right] \bar{u}_{\bar{x}}+\psi_{u}^{2} \xi_{x} L_{201 A}(u)}
$$

and allowing a new branch on $L_{201 A}$ :

5.3.1.1 Case A-1-1: $L_{200 A}(u)=0, L_{201 A}(u)=0$

We have

$$
\bar{L}_{2}=\frac{\chi^{\prime}}{\psi_{u} \tilde{\zeta}_{x}^{2} L_{202 A}(u) \overline{\mathcal{u}}_{\bar{x}}}
$$

and

$$
\mathcal{L}:=\frac{1}{2} \frac{1}{L_{202 A}(u) u_{x}} u_{t}^{2}+L_{10 A}\left(u_{x}, u\right) u_{t}+\frac{u_{x x}^{2}}{L_{021}(u)^{4} u_{x}^{5} L_{202 A}(u)}+L_{00 A}\left(u_{x}, u\right)
$$

which is further simplified with $L_{202 A}(u):=1$ to

$$
\mathcal{L}:=\frac{1}{2} \frac{1}{u_{x}} u_{t}^{2}+L_{10 A}\left(u_{x}, u\right) u_{t}+\frac{u_{x x}^{2}}{L_{021}(u)^{4} u_{x}^{5}}+L_{00 A}\left(u_{x}, u\right)
$$


The next obstruction comes from coefficient of $u_{x x} u_{t}$ on $d_{1}$ and determines the form of above mentioned $L_{10 A}\left(u_{x}, u\right)$ as

$$
L_{10 A}\left(u_{x}, u\right):=\frac{B(u) u_{x}+C(u)}{u_{x}^{2}}
$$

after integration of equation

$$
6 L_{10}\left(u_{x}, u\right)_{u_{x}}+6 L_{10}\left(u_{x}, u\right)_{u_{x} u_{x}} u_{x}+u_{x}^{2} L_{10}\left(u_{x}, u\right)_{u_{x} u_{x} u_{x}}=0
$$

and setting of $A(u)=0$ in its solution as per Lagrangian equivalence. Next we find the following obstruction from coefficient on $u_{t}$ of $d_{1}$

$$
L_{021}(u) C(u)_{u}+2 C(u) L_{021}(u)_{u}=0
$$

solved by

$$
C(u):=\frac{\gamma}{L_{021}(u)^{2}}, \gamma \in \mathbf{C}
$$

Then we proceed by looking for new obstructions coming from canonical conservation laws $d_{3}$ and $d_{5}$. After setting of the jet variables on $\sigma_{3}$ the coefficient on $u_{x x} u_{t}$ of $d_{3}$ yields the following differential equation on $L_{00 A}\left(u_{x}, u\right)$

$$
\begin{aligned}
-12 \gamma^{2}+12 L_{021}(u)^{4} u_{x}^{5} L_{00 A}\left(u_{x}, u\right)_{u_{x} u_{x}} & + \\
8 L_{021}(u)^{4} u_{x}^{6} L_{00 A}\left(u_{x}, u\right)_{u_{x} u_{x} u_{x}} & + \\
L_{021}(u)^{4} u_{x}^{7} L_{00 A}\left(u_{x}, u\right)_{u_{x} u_{x} u_{x} u_{x}} & =0
\end{aligned}
$$

which is solved by

$$
L_{00 A}\left(u_{x}, u\right):=L_{000}(u)+\frac{\gamma^{2}}{2 L_{021}(u)^{4} u_{x}^{3}}+\frac{L_{0002}(u)}{u_{x}^{2}}+\frac{L_{0001}(u)}{u_{x}}+L_{001}(u) u_{x}
$$

and $L_{001}(u):=0$ per Lagrangian equivalence $\left(L_{001}(u) u_{x}\right.$ being a total derivative). After these settings a new obstruction from coefficient of $d_{3}$ on $u_{x}^{2} u_{t}$,

$$
\gamma^{2} L_{021}(u)_{u}=0
$$

opens two new branches:

- $\gamma=0$,with the following set of subsequent obstructions and restrictions

$$
\begin{aligned}
L_{021}(u) L_{0002}(u)_{u}+3 L_{021}(u)_{u} L_{0002}(u) & =0, & & L_{0002}(u):=\frac{\delta}{L_{021}(u)^{3}} \\
L_{021}(u) B(u)_{u}+2 L_{021}(u)_{u} B(u) & =0, & & B(u):=\frac{\beta}{L_{021}(u)^{2}} \\
\delta_{1}^{2} L_{021}(u)_{u} & =0 & &
\end{aligned}
$$

being the last obstruction a new fork, leading to our two first integrable cases: 
1. For $\delta=0$

$$
\begin{aligned}
\mathcal{L}_{A 1} & =\frac{u_{t}^{2}}{2 u_{x}}+\frac{u_{x x}^{2}}{L_{021}(u)^{4} u_{x}^{5}} \\
& +\frac{\beta}{L_{021}(u)^{2} u_{x}} u_{t}+\frac{L_{0001}(u)}{u_{x}}+L_{000}(u)
\end{aligned}
$$

2. For $L_{021}(u)=\lambda$,

$$
\begin{aligned}
\mathcal{L}_{A 2} & =\frac{u_{t}^{2}}{2 u_{x}}+\frac{u_{x x}^{2}}{\lambda^{4} u_{x}^{5}}+\frac{\delta_{1}}{\lambda^{3} u_{x}^{2}} \\
& +\frac{\beta}{\lambda^{2} u_{x}} u_{t}+\frac{L_{0001}(u)}{u_{x}}+L_{000}(u) \\
\delta_{1} & \neq 0
\end{aligned}
$$

- $\gamma \neq 0, L_{021}(u)=\lambda$ with obstructions and restrictions from canonical conservation laws $d_{3}$ and $d_{5}$

$$
\begin{array}{rlrl}
\lambda\left(-\gamma B(u)_{u}+\lambda^{2} L_{0002}(u)_{u}\right) & =0, & & L_{0002}(u):=\frac{\gamma b(u)}{\lambda}+\delta_{1} \\
\gamma \delta_{1} \lambda^{6}=0, & & \delta_{1}:=0 \\
3 B(u) B(u)_{u}-2 L_{0000}(u) & =0, & & L_{0000}(u):=\frac{3}{4} B(u)^{2}+\delta_{2}
\end{array}
$$

leading to the third integrable case

$$
\begin{aligned}
\mathcal{L}_{A 3} & =\frac{u_{t}^{2}}{2 u_{x}}+\frac{\gamma+\lambda^{2} B(u) u_{x}}{\lambda^{2} u_{x}^{2}} u_{t} \\
& +\frac{u_{x x}^{2}}{\lambda^{4} u_{x}^{5}}+\frac{\gamma^{2}}{2 \lambda^{4} u_{x}^{3}}+\frac{\gamma B(u)}{\lambda^{2} u_{x}^{2}}+\frac{4 \delta_{2}+3 B(u)^{2}}{4 u_{x}} \\
& +\frac{\delta_{3} \lambda^{2} B(u)}{\gamma}+\frac{\lambda^{2} B(u)^{3}}{4 \gamma}-\frac{B(u)_{u u}}{\gamma \lambda^{2}}+\delta_{3}
\end{aligned}
$$

5.3.1.2 Case A-1-2: $L_{200 A}(u)=0, L_{201 A}(u) \neq 0$

When $L_{201 A}(u) \neq 0$ we can set $L_{200 A}(u)=0$ and

$$
\begin{aligned}
t & \rightarrow t \\
x & \rightarrow x+\int L_{202 A}(u) d u \\
u & \rightarrow u
\end{aligned}
$$

and $L_{201 A}(u)=1$ in order to simplify coefficients on $u_{t}^{2}$ and $u_{x x}$, resulting in

$$
\mathcal{L}=\frac{u_{t}^{2}}{2}+L_{10 A}\left(u_{x}, u\right) u_{t}+\frac{u_{x x}^{2}}{u_{x}^{4}}+L_{00 A}\left(u_{x}, u\right)
$$


Second canonical conservation law gives now on its $u_{x x}^{2} u_{t}$ coefficient

$$
3 \frac{\partial^{2} L_{10 A}}{\partial u_{x}^{2}}+u_{x} \frac{\partial^{3} L_{10 A}}{\partial u_{x}^{3}}=0
$$

thus we set

$$
L_{10 A}\left(u_{x}, u\right):=\frac{C(u)}{u_{x}}+A(u) u_{x}
$$

and find as coefficient of $u_{x x}^{2}$ and $u_{t}$ in $d_{1}$

$$
\begin{aligned}
L_{021}(u) & :=\lambda \in \mathbf{C} \\
C(u) & :=\in \mathbf{C}
\end{aligned}
$$

and $u_{x x}^{2} u_{t}$ in $d_{5}$

$$
\begin{array}{r}
9 \gamma^{2}+3 A(u)^{2} u_{x}^{4}-3 u_{x}^{4} \frac{\partial^{2} L_{00 A}}{\partial u_{x}^{2}}+ \\
-5 u_{x}^{5} \frac{\partial^{3} L_{00}}{\partial u_{x}^{3}}-u_{x}^{6} \frac{\partial^{4} L_{00 A}}{\partial u_{x}^{4}}=0
\end{array}
$$

which is solved by

$$
\begin{aligned}
L_{00 A}\left(u_{x}, u\right) & :=L_{000}(u)+\frac{\gamma^{2}}{2 u_{x}^{2}}+\frac{L_{0001}(u)}{u_{x}}+L_{001}(u) u_{x} \\
& +L_{002}(u)\left(u_{x}-u_{x} \log u_{x}\right)+\frac{A(u)^{2} u_{x}^{2}}{8}
\end{aligned}
$$

where we can neglect term $L_{001}(u) u_{x}$ being a total derivative.

Now coefficient on $u_{t}$ of $d_{3}$ is

$$
\gamma \lambda^{3} A(u)_{u}
$$

which opens a new fork with two cases (as $L_{021}(u):=\lambda \neq 0$ for all sub-cases in case A):

- $\gamma=0$, with $\lambda^{3} L_{0001}(u)_{u}=0, L_{0001}(u):=\lambda \in \mathbf{C}$, and a new fork by

$$
\delta \lambda^{4} A(u)_{u}=0
$$

with 2 new sub-cases

1. $\delta=0$ makes

$$
\begin{aligned}
\mathcal{L}_{A 4} & =\frac{u_{t}^{2}}{2}+\frac{u_{x x}^{2}}{\lambda^{4} u_{x}^{4}}+\frac{A(u)^{2}}{8} u_{x}^{2} \\
& +\frac{A(u)}{2} u_{t} u_{x}+L_{002}(u) u_{x}\left(1-\log u_{x}\right) \\
& +L_{000}(u)
\end{aligned}
$$


2. $\delta \neq 0, A(u):=\in \mathbf{C}$, from coefficient of $u_{x} \log u_{x}$ in $d_{5}$ we have that

$$
\begin{aligned}
& L_{002}(u):=\delta_{2} \in \mathbf{C} \\
& L_{000}(u):=\delta_{0} \in \mathbf{C}
\end{aligned}
$$

and

$$
\begin{aligned}
\mathcal{L}_{A 5} & =\frac{u_{t}^{2}}{2}+\frac{u_{x x}^{2}}{\lambda^{4} u_{x}^{4}}+\frac{\alpha^{2}}{8} u_{x}^{2} \\
& +\frac{\alpha}{2} u_{x} u_{t}-\delta_{2} u_{x} \log u_{x}+\frac{\delta}{u_{x}}+\delta_{0}
\end{aligned}
$$

- $\gamma \neq 0$, with $A(u)=\alpha \in \mathbf{C}$, the following obstructions are integrated:

$$
\begin{aligned}
\lambda^{3} L_{0001}(u)_{u}=0, & L_{0001}(u):=\delta_{3} \\
\gamma \lambda^{3} L_{002}(u)_{u}=0, & L_{002}(u):=\delta_{2} \\
\gamma \lambda^{3} L_{000}(u)_{u}=0, & L_{000}(u):=\delta_{0} \\
\gamma \lambda^{4} \delta_{2}=0, & \delta_{2}:=0
\end{aligned}
$$

resulting in

$$
\begin{aligned}
\mathcal{L}_{A 6} & =\frac{u_{t}^{2}}{2}+\frac{u_{x x}^{2}}{\lambda^{4} u_{x}^{4}}+\frac{\gamma^{2}}{2 u_{x}^{2}} \\
& +\left(\frac{\gamma}{u_{x}}+\frac{\alpha}{2} u_{x}\right) u_{t}+\frac{\delta_{3}}{u_{x}}+\frac{\alpha^{2}}{8} u_{x}^{2}+\delta_{0}
\end{aligned}
$$

5.3.2 Case A-2: $L_{200 A}(u) \neq 0$.

$L_{201 A}(u):=0$ is permitted as above to study Lagrangian densities of the form

$$
\begin{aligned}
\mathcal{L}_{A} & :=\frac{1}{2} \frac{u_{x}}{L_{202 A}(u) u_{x}^{2}+L_{200 A}(u)} u_{t}^{2} \\
& +L_{10 A}\left(u_{x}, u\right) u_{t} \\
& +\frac{u_{x x}^{2}}{L_{021}(u)^{4} u_{x}^{3}\left(L_{202 A}(u) u_{x}^{2}+L_{200 A}(u)\right)} \\
& +L_{00 A}\left(u_{x}, u\right)
\end{aligned}
$$


Obstructions from $d_{1}$ on $u_{x x}^{2}$ are

$$
\begin{aligned}
L_{021}(u) L_{200 A}(u)_{u}+3 L_{021}(u)_{u} L_{200 A}(u) & =0, \\
3 L_{021}(u) L_{202 A}(u) L_{200 A}(u)_{u}+L_{021}(u) L_{200 A}(u) L_{202 A}(u)_{u} & + \\
10 L_{021}(u)_{u} L_{202 A}(u) L_{200}(u) & =0
\end{aligned}
$$

yielding

$$
\begin{aligned}
& L_{200 A}(u):=\frac{K_{200 A}}{L_{021}(u)^{3}}, K_{200 A} \neq 0 \\
& L_{202 A}(u):=\frac{K_{202 A}}{L_{021}(u)}, K_{200 A} \in \mathbf{C}
\end{aligned}
$$

which can be transformed by $L_{021}(u):=1$ to

$$
\begin{aligned}
\mathcal{L} & :=\frac{1}{2} \frac{u_{x}}{K_{202 A} u_{x}^{2}+K_{200 A}} u_{t}^{2}+L_{10 A}\left(u_{x}, u\right) u_{t} \\
& +\frac{u_{x x}^{2}}{u_{x}^{3}\left(K_{202 A} u_{x}^{2}+K_{200 A}\right)}+L_{00 A}\left(u_{x}, u\right)
\end{aligned}
$$

from coefficient of $u_{x x} u_{t}$ in $d_{1}$ we can set

$$
L_{10 A}\left(u_{x}, u\right):=\frac{\frac{1}{2} A(u) u_{x}^{2}+B(u) u_{x}+C(u)}{K_{202 A} u_{x}^{2}+K_{200 A}}
$$

and from the rest of coefficients in $d_{1}$

$$
\begin{aligned}
A(u) & :=\frac{2 K_{202 A}}{K_{200 A}} C(u)+\alpha \\
B(u) & :=\beta
\end{aligned}
$$

with $\alpha, \beta \in \mathbb{C}$ and

$$
L_{00}\left(u_{x}, u\right):=L_{000}\left(u_{x}\right)
$$

after integration of

$$
K_{200 A} \frac{\partial L_{00}\left(u_{x}, u\right)}{\partial u_{x}^{2} u}=0
$$

and elimination of total derivatives in its general solution.

We branch now on two sub-cases depending on $K_{202 A}$ vanishing or not.

5.3.2.1 Case A-2-1: $L_{200 A}(u) \neq 0, K_{202 A}=0$

Obstructions on $d_{3}, u_{x x} u_{t}$ and $d_{5}, u_{x x}^{3}$ have respectively fourth and fifth order equations on $L_{000}\left(u_{x}\right)$ and can be conveniently combined to obtain a single obstruction with an ODE of the fourth order

$$
288 \alpha^{2}-384 K_{200 A} L_{000}^{\prime \prime \prime}\left(u_{x}\right)-96 K_{200 A} L_{000}^{\prime \prime \prime \prime}\left(u_{x}\right) u_{x}=0
$$


with solution, after elimination of total derivatives

$$
L_{000}\left(u_{x}\right):=\frac{L_{0000} u_{x}^{2}}{576 K_{200 A}}+\frac{L_{0003}}{96 K_{200 A} u_{x}}+\frac{\alpha^{2} u_{x}{ }^{3}}{8 K_{200 A}}
$$

yielding

$$
\begin{aligned}
\mathcal{L}_{A 7} & =\frac{u_{x}}{2 K_{200 A}} u_{t}^{2}+\frac{\beta u_{x}+\frac{\alpha u_{x}^{2}}{2}}{K_{200 A}} u_{t}+\frac{u_{x x}^{2}}{K_{200 A} u_{x}^{3}} \\
& +\frac{\alpha^{2} u_{x}^{3}}{8 K_{200 A}}+\frac{\alpha \beta u_{x}^{2}}{2 K_{200 A}}+\frac{L_{0003}}{96 K_{200 A} u_{x}}
\end{aligned}
$$

5.3.2.2 Case A-2-2: $L_{200 A}(u) \neq 0, K_{202 A} \neq 0$

Like in the previous case, obstructions on $d_{3}, u_{x x} u_{t}$ and $d_{5}, u_{x x}^{3}$ have respectively fourth and fifth order on $L_{000}\left(u_{x}\right)$ and can be combined to obtain a single obstruction of the fourth order, resulting in

$$
\begin{aligned}
\mathcal{L}_{A 8} & =\frac{u_{x}}{2\left(K_{200 A}+K_{202 A} u_{x}^{2}\right)} u_{t}^{2}+\frac{u_{x x}^{2}}{u_{x}^{3}\left(K_{200 A}+K_{202 A} u_{x}^{2}\right)} \\
& +\frac{\beta u_{x}+\frac{\alpha u_{x}^{2}}{2}}{K_{200 A}+K_{202 A} u_{x}^{2}} u_{t} \\
& +\frac{K_{200 A} \alpha^{2}-4 K_{202 A}\left(L_{0001} K_{202 A}+\beta^{2}\right)}{8 K_{202 A}^{2} u_{x}} \\
& +\frac{-4 K_{200 A} \alpha \beta+u_{x}\left(4 K_{202 A} \beta^{2}-K_{200 A} \alpha^{2}\right)}{8 K_{202 A}\left(K_{200 A}+K_{202 A} u_{x}^{2}\right)}
\end{aligned}
$$

5.4 CASE в: $K_{020} \neq 0, L_{021}(u)=0$.

We have in this second main case

$$
\begin{aligned}
\mathcal{L}_{B} & :=\frac{1}{2} \frac{1}{L_{202}(u) u_{x}^{2}+L_{201}(u) u_{x}+L_{200}(u)} u_{t}^{2} \\
& +L_{10}\left(u_{x}, u\right) u_{t} \\
& +\frac{u_{x x}^{2}}{L_{202}(u) u_{x}^{2}+L_{201}(u) u_{x}+L_{200}(u)} \\
& +L_{00}\left(u_{x}, u\right)
\end{aligned}
$$

obtained from 5.2 .18 by setting $K_{020}:=1$ and $L_{021}(u):=0$.

Now from $d_{1}$ coefficient's on $u_{x x}^{2} u_{t}^{2} u_{x}^{3}$ we have

$$
L_{202}(u) \frac{\partial L_{202}(u)}{\partial u}=0
$$


and thus we set $L_{202}(u):=K_{202}$ as the most generic solution (allowing $L_{202}(u)$ to vanish), to this obstruction and extract new obstructions from $u_{x x}^{2} u_{t}, 5.2 .20$ becomes

$$
L_{10}\left(u_{x}, u\right):=\frac{\frac{1}{2} A(u) u_{x}^{2}+B(u) u_{x}+C(u)}{K_{202}(u) u_{x}^{2}+L_{201}(u) u_{x}+L_{200}(u)},
$$

making

$$
\begin{aligned}
\mathcal{L}_{B} & :=\frac{1}{2} \frac{1}{K_{202}(u) u_{x}^{2}+L_{201}(u) u_{x}+L_{200}(u)} u_{t}^{2} \\
& +\frac{\frac{1}{2} A(u) u_{x}^{2}+B(u) u_{x}+C(u)}{K_{202}(u) u_{x}^{2}+L_{201}(u) u_{x}+L_{200}(u)} u_{t} \\
& +\frac{u_{x x}^{2}}{K_{202}(u) u_{x}^{2}+L_{201}(u) u_{x}+L_{200}(u)} \\
& +L_{00}\left(u_{x}, u\right)
\end{aligned}
$$

being the next obstruction (on $d_{1}, u_{x x}^{2} u_{t}^{2}$ )

$$
2 L_{200}(u) \frac{\partial L_{201}(u)}{\partial u}-L_{201}(u) \frac{\partial L_{200}(u)}{\partial u}=0
$$

that imposes

$$
L_{200}(u):=K_{2000} L_{201}(u)^{2}
$$

which accounts for another possible branch. According to allowed point transformations at this stage of the Lagrangian we fork at this point on the values of $K_{202}$, with sub-cases depending on $L_{201}(u)$.

\subsubsection{Case $B-1: K_{202}:=0$.}

Analyzing obstructions after setting $K_{202}:=0$ we find sub-cases depending on $L_{201}(u)$ :

5.4.1.1 Case B-1-1: $L_{201}(u)=0$.

5.4. I becomes in this sub-case

$$
\mathcal{L}:=\frac{1}{2} \frac{1}{L_{200}(u)} u_{t}^{2}+\frac{\frac{1}{2} A(u) u_{x}^{2}+B(u) u_{x}+C(u)}{L_{200}(u)} u_{t}+\frac{u_{x x}^{2}}{L_{200}(u)}+L_{00}\left(u_{x}, u\right)
$$

which can be transformed by

$$
\begin{aligned}
\xi & =\xi(x) \\
\psi & =\psi(x, u)
\end{aligned}
$$

to

$$
\bar{L}_{2}=\frac{1}{\xi_{x} \psi_{u}^{2} L_{200}(u)}
$$


allowing $L_{200}:=1$ and giving equivalent Lagrangian

$$
\mathcal{L}:=\frac{1}{2} u_{t}^{2}+\left(\frac{1}{2} A(u) u_{x}^{2}+B(u) u_{x}+C(u)\right) u_{t}+u_{x x}^{2}+L_{00}\left(u_{x}, u\right) .
$$

where term $C(u) u_{t}$ can in turn be neglected being a total derivative. Obstruction on $d_{1}, u_{t}$ forces $B(u):=\beta \in \mathbf{C}$ and the one in $d_{3}, u_{x x} u_{t}$ consist of differential equation

$$
3 A(u)-\frac{\partial^{4} L_{00}}{\partial u_{x}^{4}}=0
$$

with solution

$$
L_{00}\left(u_{x}, u\right)=L_{000}(u)+L_{001}(u) u_{x}+\frac{1}{2} L_{002}(u) u_{x}^{2}+\frac{1}{6} L_{003}(u) u_{x}^{3}+\frac{1}{8} A(u)^{2} u_{x}^{4}
$$

where again by Lagrangian equivalence $L_{001}(u):=0$. After these settings, obstructions from $d_{3}$ imply

$$
\begin{aligned}
A(u):=\alpha & \in \mathbf{C}, \\
\alpha L_{002}(u)_{u} & =0, \\
L_{003}(u)_{u} & =0, \\
\alpha L_{000}(u)_{u} & =0,
\end{aligned}
$$

exhausting conditions from $d_{3}$. On $d_{5}$ we find $\alpha\left(\alpha \beta-L_{003}(u)\right)=0$, making Lagrangian

$\mathcal{L}:=\frac{u_{t}^{2}}{2}+\left(\frac{\alpha u_{x}^{2}}{2}+\beta u_{x}\right) u_{t}+u_{x x}^{2}+\frac{\alpha^{2}}{8} u_{x}^{4}+\frac{K_{003}}{6} u_{x}^{3}+\frac{K_{002}}{2} u_{x}^{2}+L_{000}(u)$

with aditional obstructions

$$
\begin{aligned}
\alpha L_{000}^{\prime}(u) & =0, \\
K_{003} & =3 \alpha \beta \\
\left(K_{003}-5 \alpha \beta-2 \alpha s_{0}\right) L_{000}^{\prime}(u) & =0
\end{aligned}
$$

providing three sub-cases

1. $\alpha:=0, K_{003}:=0$ makes

$$
\mathcal{L}_{B 1}:=\frac{u_{t}^{2}}{2}+\beta u_{x} u_{t}+u_{x x}^{2}+\frac{K_{002}}{2} u_{x}^{2}+L_{000}(u)
$$

2. $\alpha:=0, K_{003} \neq 0$

$$
\begin{aligned}
\mathcal{L}_{B 2} & :=\frac{u_{t}^{2}}{2}+\beta u_{x} u_{t}+u_{x x}^{2} \\
& +\frac{K_{003}}{6} u_{x}^{3}+\frac{K_{002}}{2} u_{x}^{2}, \quad K_{003} \neq 0
\end{aligned}
$$


3. $\alpha \neq 0$

$$
\begin{aligned}
\mathcal{L}_{B 3} & :=\frac{u_{t}^{2}}{2}+\left(\frac{1}{2} \alpha u_{x}^{2}+\beta u_{x}\right) u_{t}+u_{x x}^{2} \\
& +\frac{1}{8} \alpha^{2} u_{x}^{4}++\frac{\alpha \beta}{2} u_{x}^{3}+\frac{\delta_{2}}{2} u_{x}^{2}, \quad \alpha \neq 0
\end{aligned}
$$

5.4.1.2 Case B-1-2: $L_{201}(u) \neq 0$.

5.4 .3 forces the value of $L_{200}(u)$ in this case and $L_{2}$ can be transformed to

$$
\bar{L}_{2}:=\frac{1}{\xi_{x} \psi_{u}} \frac{1}{L_{201}(u) \xi_{x} \bar{u}_{\bar{x}}-L_{201}(u) \psi_{x}+K_{2000} L_{201}(u)^{2} \psi_{u}}
$$

by a point transformation with

$$
\begin{aligned}
\xi(x) & =x \\
\psi^{\prime}(u) & =\frac{1}{L_{201}(u)}
\end{aligned}
$$

which can be normalized to

$$
\bar{L}_{2}:=\frac{1}{\bar{u}_{\bar{x}}+K_{2000}}
$$

thus justifing $L_{201}:=1$. Obstruction on $d_{1}, u_{t} u_{x}^{2}$ implies that $A(u)=$ $\alpha \in \mathbf{C}$ and on $u_{t} u_{x}$ that $C(u)=K_{2000} B(u)+\gamma$. The rest of $d_{1}$ gives a PDE with solution equivalent to

$$
L_{00}\left(u_{x}, u\right):=L_{000}\left(u_{x}\right)
$$

Lagrangian at this point can be further simplified by neglecting term $B(u) u_{t}$ as per Lagrangian equivalence

$$
\mathcal{L}=\frac{u_{t}^{2}}{2\left(K_{2000}+u_{x}\right)}+\frac{u_{x x}^{2}}{K_{2000}+u_{x}}+\frac{\gamma+\frac{1}{2} \alpha u_{x}^{2}}{K_{2000}+u_{x}}+L_{000}\left(u_{x}\right)
$$

The form of $L_{000}\left(u_{x}\right)$ is determined by the the equation from the coefficient of $u_{x x} u_{t}$ in $d_{3}$

$$
\begin{aligned}
L_{000}\left(u_{x}\right):= & \frac{\alpha^{2} K_{2000} 4}{8\left(K_{2000}+u_{x}\right)}-\frac{3 \alpha^{2} K_{2000}^{3}+L_{0000}}{8} \log \left(K_{2000}+u_{x}\right) \\
& +\frac{\alpha \gamma K_{2000}^{2}}{2\left(K_{2000}+u_{x}\right)}+\frac{\gamma^{2}}{2\left(K_{2000}+u_{x}\right)}-\frac{L_{0001} u_{x}^{2}}{8}+\frac{\alpha^{2} u_{x}^{3}}{8}
\end{aligned}
$$

the rest of $d_{5}$ implies

$$
\alpha\left(3 \alpha^{2} K_{2000}{ }^{3}+L_{0000}\right)=0
$$

So we have two cases depending on $\alpha$ 
- $\alpha=0$. No more obstructions exist and resulting Lagrangian is

$$
\begin{aligned}
\mathcal{L}_{B 4} & :=\frac{u_{t}^{2}}{2\left(K_{2000}+u_{x}\right)}+\frac{u_{x x}^{2}}{K_{2000}+u_{x}} \\
& +\frac{\gamma^{2}}{2\left(K_{2000}+u_{x}\right)}+\frac{\gamma}{K_{2000}+u_{x}} u_{t} \\
& +\frac{L_{0001} u_{x}^{2}}{8}+\frac{L_{0000}}{8} \log \left(K_{2000}+u_{x}\right)
\end{aligned}
$$

- $\alpha \neq 0$. From $d_{5}, u_{x x} u_{t}$ we have $L_{0000}=-3 K_{2000}^{3} \alpha^{2}$ and

$$
\begin{aligned}
\mathcal{L}_{B 5} & :=\frac{u_{t}^{2}}{2\left(K_{2000}+u_{x}\right)}+\frac{u_{x x}^{2}}{K_{2000}+u_{x}}+\frac{\gamma+\frac{1}{2} \alpha u_{x}^{2}}{K_{2000}+u_{x}} u_{t} \\
& +\frac{\gamma^{2}}{2\left(K_{2000}+u_{x}\right)}+\frac{L_{0001} u_{x}^{2}}{8}+\frac{1}{8} \alpha^{2} u_{x}^{3}
\end{aligned}
$$

\subsubsection{Case $B-2: K_{202} \neq 0$.}

According to 5.4.2 and 5.4.3 we have two branches depending on $L_{201}(u)$.

\subsubsection{Case B-2-1: $L_{201}(u)=0$.}

Point transformation for case B forces two branches depending on $L_{200}(u)$.

1. $L_{200}(u):=0$ permits $K_{202}:=1$ and

$$
\mathcal{L}=\frac{u_{t}^{2}}{2 u_{x}^{2}}+\frac{u_{x x}^{2}}{u_{x}^{2}}+\frac{\frac{1}{2} A(u) u_{x}^{2}+B(u) u_{x}+C(u)}{u_{x}^{2}} u_{t}+L_{00}\left(u_{x}, u\right)
$$

Obstruction on $d_{1}, u_{t} u_{x x}$ implies that $B(u)=\beta \in \mathbb{C}$, and the one on $d_{3}, u_{t} u_{x x}$ contains differential equation

$$
12 C(u)^{2}+12 \beta C(u) u_{x}+6 u_{x}^{5} \frac{\partial^{3} L_{00}\left(u_{x}, u\right)}{\partial u_{x}^{3}}+u_{x}^{6} \frac{\partial^{4} L_{00}\left(u_{x}, u\right)}{\partial u_{x}^{4}}=0
$$

with equivalent solution

$$
L_{00}\left(u_{x}, u\right):=\frac{C(u)^{2}}{2 u_{x}{ }^{2}}+\frac{\beta C(u)}{u_{x}}+\frac{L_{0003}(u)}{u_{x}{ }^{3}}+L_{002}(u) u_{x}^{2}+L_{000}(u)
$$

while the coefficient of $u_{t}$ in $d_{3}$ makes $L_{000}(u)=K_{000}$ and that of $u_{x x} u_{t}^{2}$ in $d_{5} L_{0003}(u)=0$ and finally, $d_{3}$ implies that

$$
2 L_{002}(u) c^{\prime}(u)+c(u) L_{002}^{\prime}(u)+20 L_{002}^{\prime \prime \prime}(u)=0 \text { (5.4.4) }
$$

Now we have sub-cases depending on $C(u)$ 
- For $C(u)=0$ the Lagrangian is equivalent to

$$
\mathcal{L}_{B 6}:=\frac{u_{t}^{2}}{2 u_{x}^{2}}+\frac{u_{x x}^{2}}{u_{x}^{2}}+\frac{\beta}{u_{x}} u_{t}+L_{002}(u) u_{x}^{2}
$$

and there are no more obstructions.

- For $C(u) \neq 0$, obstruction $5 \cdot 4 \cdot 4$ does not vanish, providing additional constrains to $L_{002}(u)$ once integrated

$$
L_{002}(u):=\frac{K_{002}-10 C^{\prime}(u)^{2}+20 C(u) C^{\prime \prime}(u)}{C(u)^{2}}
$$

and finally

$$
\begin{aligned}
\mathcal{L}_{B 7} & :=\frac{u_{t}^{2}}{2 u_{x}^{2}}+\frac{u_{x x}^{2}}{u_{x}^{2}}+\frac{C(u)+\beta u_{x}}{u_{x}^{2}} u_{t}+\frac{\beta C(u)}{u_{x}}+\frac{C(u)^{2}}{2 u_{x}^{2}} \\
& +\frac{\frac{K_{002}}{10}-C^{\prime}(u)^{2}+2 C(u) C^{\prime \prime}(u)}{C(u)^{2}} u_{x}^{2}
\end{aligned}
$$

2. $L_{200}(u) \neq 0$ permits $K_{202}:=1$ and $L_{200}(u):=-1$

Integrability conditions provide in this case $B(u):=\beta, A(u):=$ $\alpha-2 C(u)$, and

$$
\alpha \frac{\partial L_{00}\left(u_{x}, u\right)}{\partial u}:=0
$$

opening two new possibilities

- $\alpha:=0$, with new ODE obstructions in $d_{3}$ and $d_{5}$ that combine into a third order equation with solution

$$
L_{00}\left(u_{x}, u\right):=\frac{\beta^{2}}{2\left(u_{x}-1\right)}+L_{002}(u) u_{x}^{2}+L_{001}(u) u_{x}+L_{000}(u)
$$

which once set into Lagrangian produces new restrictions $L_{000}(u):=K_{000}-\frac{1}{3} L_{002}(u)$ and

$$
4 L_{002}(u) L_{002}^{\prime}(u)=L_{002}^{\prime \prime \prime}(u)
$$

whose solution is a Weierstrassian elliptic function that completes this integrable case to the form

$$
\begin{aligned}
\mathcal{L}_{B 8} & :=\frac{u_{t}^{2}}{2\left(u_{x}^{2}-1\right)}+\frac{u_{x x}^{2}}{u_{x}^{2}-1}+\frac{\beta u_{x}}{u_{x}^{2}-1} u_{t} \\
& +\frac{\beta^{2}}{2\left(u_{x}^{2}-1\right)}+L_{000}(u) u_{x}^{2}-\frac{1}{3} L_{000}(u)
\end{aligned}
$$


- $\alpha \neq 0$. From coefficient of $u_{t}$ in $d_{3}$ it follows that $\alpha \frac{\partial^{3} L_{00}\left(u_{x}, u\right)}{\partial u_{x} u_{x} u}=$ 0 , equivalently solved again by setting $L_{00}\left(u_{x}, u\right):=L_{000}\left(u_{x}\right)+$ $L_{001}(u)$ which leads to a new differential equation after combining obstructions in coefficient of $u_{x x} u_{t}$ in $d_{3}$ and of $u_{x x}^{2}$ in $d_{5}$

$$
\begin{aligned}
-24 \alpha \beta-6 \alpha^{2} u_{x}-24 \beta^{2} u_{x}+96 \alpha \beta u_{x}^{2} & + \\
+\left(36 \alpha^{2}+144 \beta^{2}\right) u_{x}^{3}-24 \alpha \beta u_{x}^{4} & + \\
-30 \alpha^{2} u_{x}^{5}-120 \beta^{2} u_{x}^{5}-48 \alpha \beta u_{x}^{6} & + \\
6\left(u_{x}^{2}-1\right)\left(-7 \alpha \beta-4 \alpha^{2} u_{x}-16 \beta^{2} u_{x}-6 \alpha \beta u_{x}^{2}\right) & + \\
6\left(u_{x}^{2}-1\right)\left(\left(2 \alpha^{2}+8 \beta^{2}\right) u_{x}^{3}+5 \alpha \beta u_{x}^{4}\right) & + \\
-6\left(u_{x}^{2}-1\right)^{5} \frac{\partial^{3} L_{000}\left(u_{x}\right)}{\partial u_{x} u_{x} u_{x}} & =0
\end{aligned}
$$

with equivalent solution

$$
L_{000}\left(u_{x}\right):=\frac{K_{0001}}{2} u_{x}^{2}+\frac{\alpha^{2}}{8\left(u_{x}^{2}-1\right)}+\frac{\beta^{2}}{2\left(u_{x}^{2}-1\right)}+\frac{\alpha \beta}{2\left(u_{x}^{2}-1\right)} u_{x}
$$

resulting in the integrable Lagrangian case

$$
\begin{aligned}
\mathcal{L}_{B 9} & :=\frac{u_{t}^{2}}{2\left(u_{x}^{2}-1\right)}+\frac{\beta u_{x}+\alpha u_{x}^{2}}{u_{x}^{2}-1}+\frac{u_{x x}^{2}}{u_{x}^{2}-1} \\
& +\frac{\alpha^{2}}{8\left(u_{x}^{2}-1\right)}+\frac{\beta^{2}}{2\left(u_{x}^{2}-1\right)}+\frac{\alpha \beta}{2\left(u_{x}^{2}-1\right)} u_{x} \\
& +\frac{K_{0001}}{2} u_{x}^{2}
\end{aligned}
$$

5.4.2.2 Case B-2-2: $L_{201}(u) \neq 0$.

Following 5.4.2 and 5.4.3 in this case we can normalize $L_{201}(u):=1$. From condition $d_{1}, u_{t} u_{x}^{4}$, we set $B(u)=\frac{1}{2 K_{202}} A(u)+\beta$, and $C(u)=$ $\frac{K_{2000}}{2 K_{202}} A(u)+\gamma$ (from $\left.d_{1}, u_{t} u_{x}^{2}\right)$. The Lagrangian is now equivalent to

$$
\begin{aligned}
\mathcal{L}:= & \frac{1}{2} \frac{1}{K_{202} u_{x}^{2}+u_{x}+K_{2000}} u_{t}^{2}+\frac{\gamma+\beta u_{x}}{K_{202} u_{x}^{2}+u_{x}+K_{2000}} u_{t} \\
& +\frac{u_{x x}^{2}}{K_{202} u_{x}^{2}+u_{x}+K_{2000}}+L_{00 A}\left(u_{x}, u\right)
\end{aligned}
$$

Integrability condition on $d_{1}$ imposes $L_{00}\left(u_{x}, u\right):=L_{001}\left(u_{x}\right)+K_{002}$. Further obstructions on $d_{3}, u_{x x} u_{t}$ and $d_{5}, u_{x x}^{3}$ yield equations of the fourth and fifth order which can be combined to a single third order equation on $L_{000}\left(u_{x}\right)$ whose solution is equivalent to

$$
L_{001}\left(u_{x}\right):=\frac{-K_{2000} \beta^{2}+K_{202} \gamma^{2}-\beta^{2} u_{x}+2 K_{202} \beta \gamma u_{x}}{2 K_{202}\left(K_{2000}+u_{x}+K_{202} u_{x}^{2}\right)}-\frac{K_{001}}{2} u_{x}^{2}
$$


being the remaining obstructions on $d_{3}$ and $d_{5}$ integrated after this setting with no more obstructions and yielding

$$
\begin{aligned}
\mathcal{L}_{B 10} & :=\frac{u_{t}^{2}}{2\left(K_{2000}+u_{x}+K_{202} u_{x}^{2}\right)}+\frac{u_{x x}^{2}}{K_{2000}+u_{x}+K_{202} u_{x}^{2}} \\
& +\frac{\gamma+\beta u_{x}}{K_{2000}+u_{x}+K_{202} u_{x}^{2}} u_{t}-\frac{K_{001}}{2} u_{x}^{2} \\
& +\frac{-K_{2000} \beta^{2}+K_{202} \gamma^{2}-\beta^{2} u_{x}+2 K_{202} \beta \gamma u_{x}}{2 K_{202}\left(K_{2000}+u_{x}+K_{202} u_{x}^{2}\right)}
\end{aligned}
$$

5.5 EQUIVALENT LAGRANGIANS UNDER POINT TRANSFORMATIONS

We have seen in the sections above that a contact transformation simplifies the expression of the generic Lagrangian 5.1.1. Furthermore, that contact transformation helps finding equivalent forms of Lagrangian which, depending on the branches derived from the integrability obstructions, result in a series of integrable cases. These cases have been derived by using only the integrability conditions or canonical densities listed in appendix. In this section we have a two-fold aim

- first, to find simpler forms of the expression of the integrable cases,

- secondly, to find equivalences between integrable cases that can be related to the same equivalent integrable case representative.

Both of this tasks can be accomplished by the use of point transformations, which preserve integrability.

\subsubsection{Equivalent form of cases representatives}

The following table summarize the geometrical or point transformations for $\mathcal{L}_{A i}, \mathcal{L}_{B i}$ found in sections 5.3, 5.4. For each case, we have

$$
\alpha, \beta, \gamma, \delta, \lambda, \epsilon \in \mathbf{C}
$$

and $a(u), b(u), \ldots$ representing arbitrary functions. Equivalent case representatives are listed in tables 5.1, 5.2, 5.3, 5.4. 


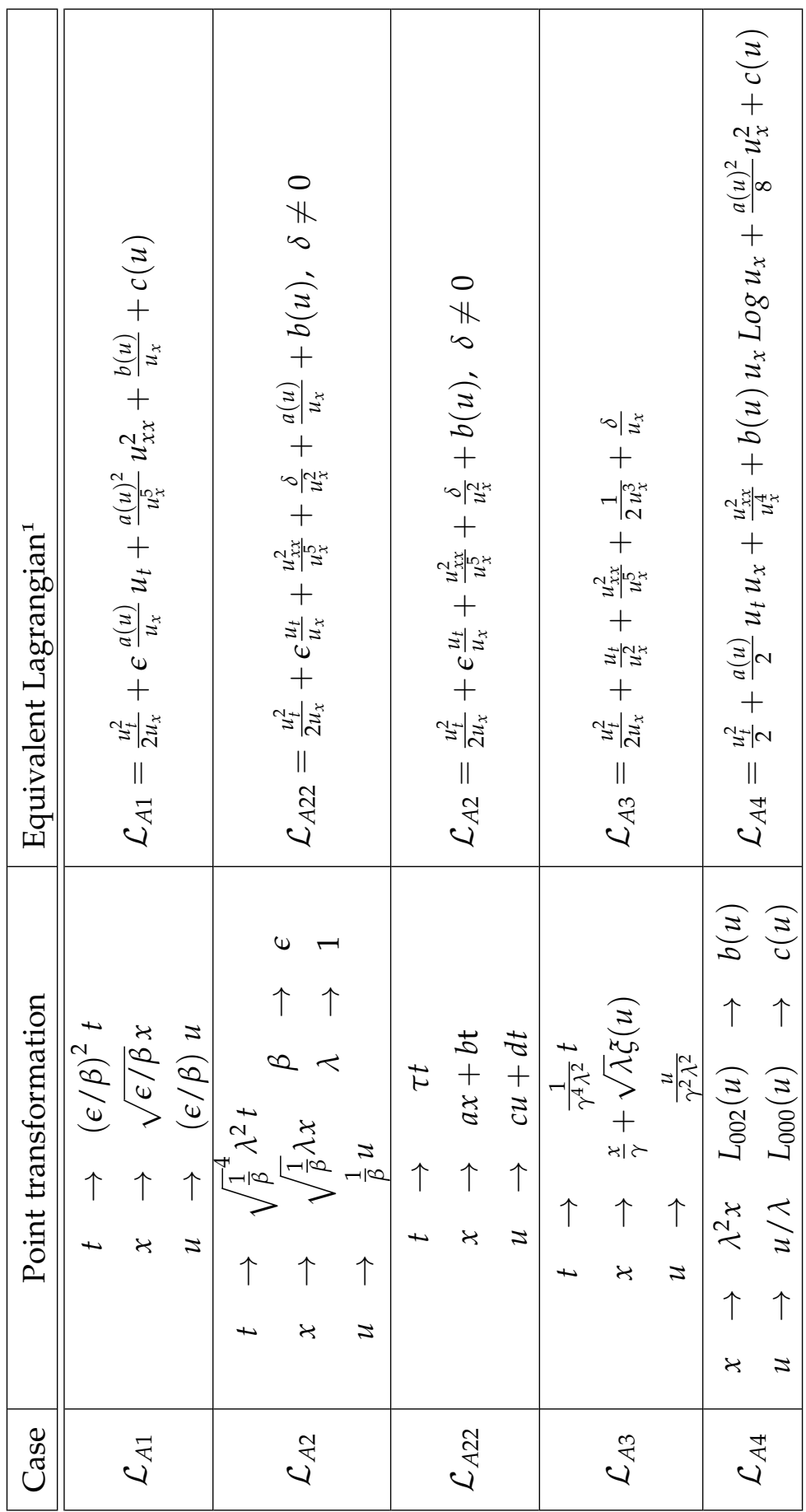

Table 5.1: Lagrangian densities after point transformations, 1 of 4

1 In the sequel we will denote with $\epsilon$ a normalization parameter with values

- $\{0,1\}$ for classifications over $\mathbf{C}$

- $\{1,-1\}$ for classifications over $\mathbf{R}$ 


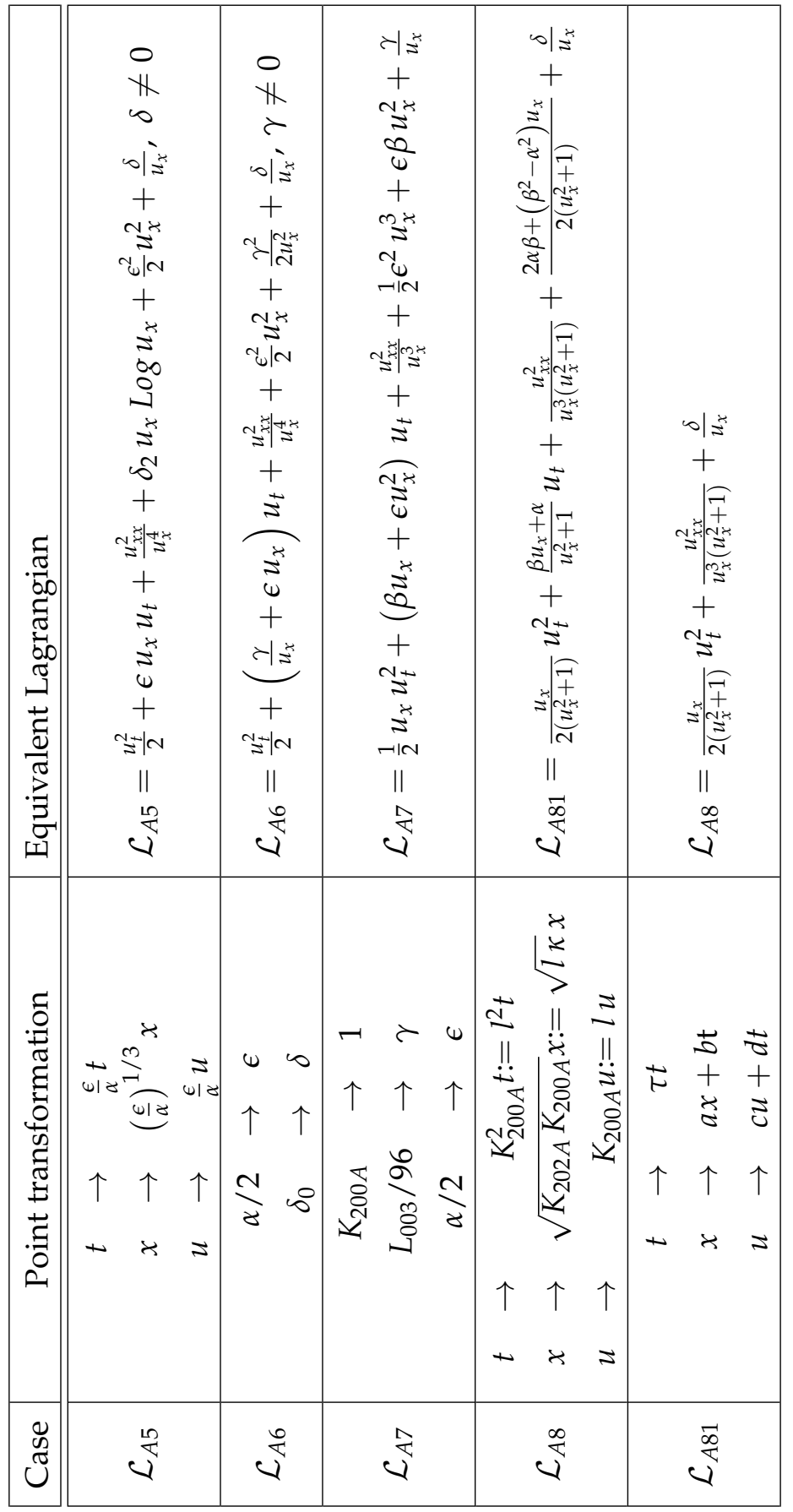

Table 5.2: Lagrangian densities after point transformations, 2 of 4 


\begin{tabular}{|c|c|c|c|c|c|}
\hline 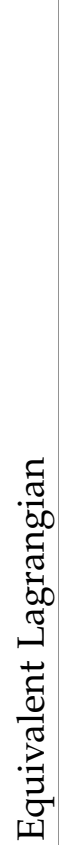 & 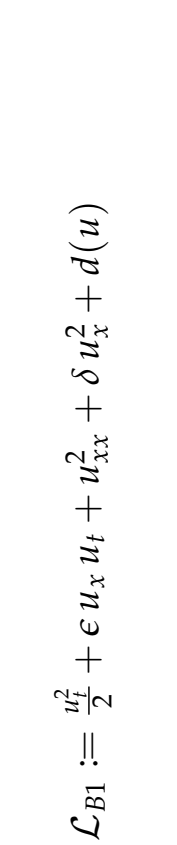 & 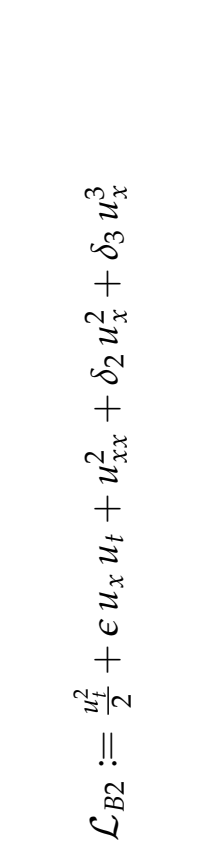 & 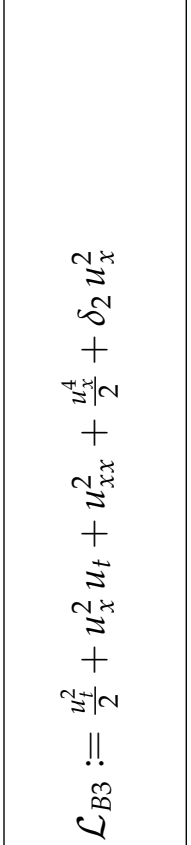 & 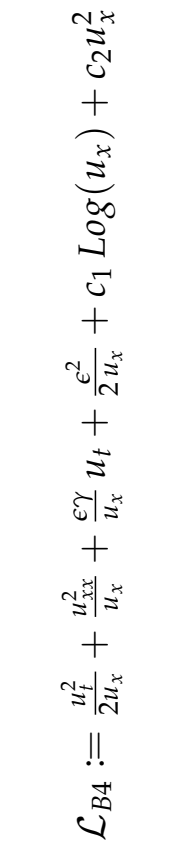 & 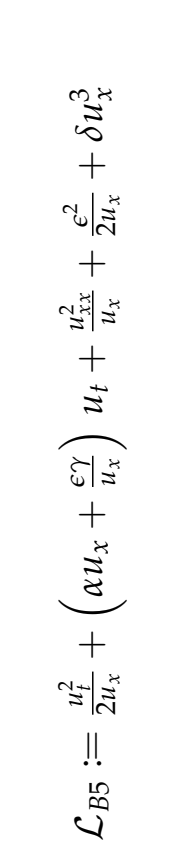 \\
\hline 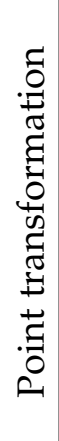 & 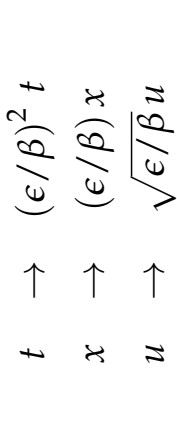 & 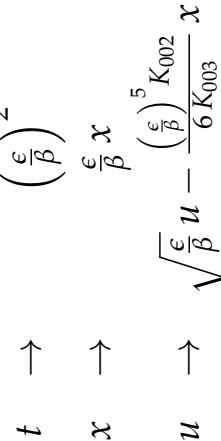 & 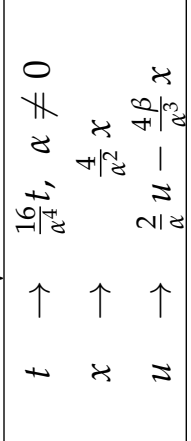 & 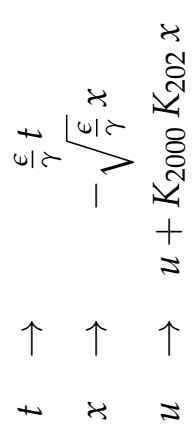 & 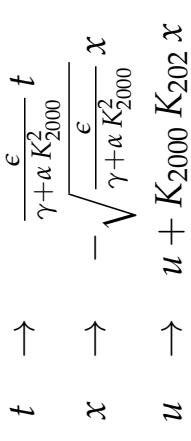 \\
\hline 岕 & $\vec{v}$ & $v^{N}$ & $\stackrel{m}{4}$ & बे & $\hat{v}^{\frac{n}{2}}$ \\
\hline
\end{tabular}

Table 5.3: Lagrangian densities after point transformations, 3 of 4 


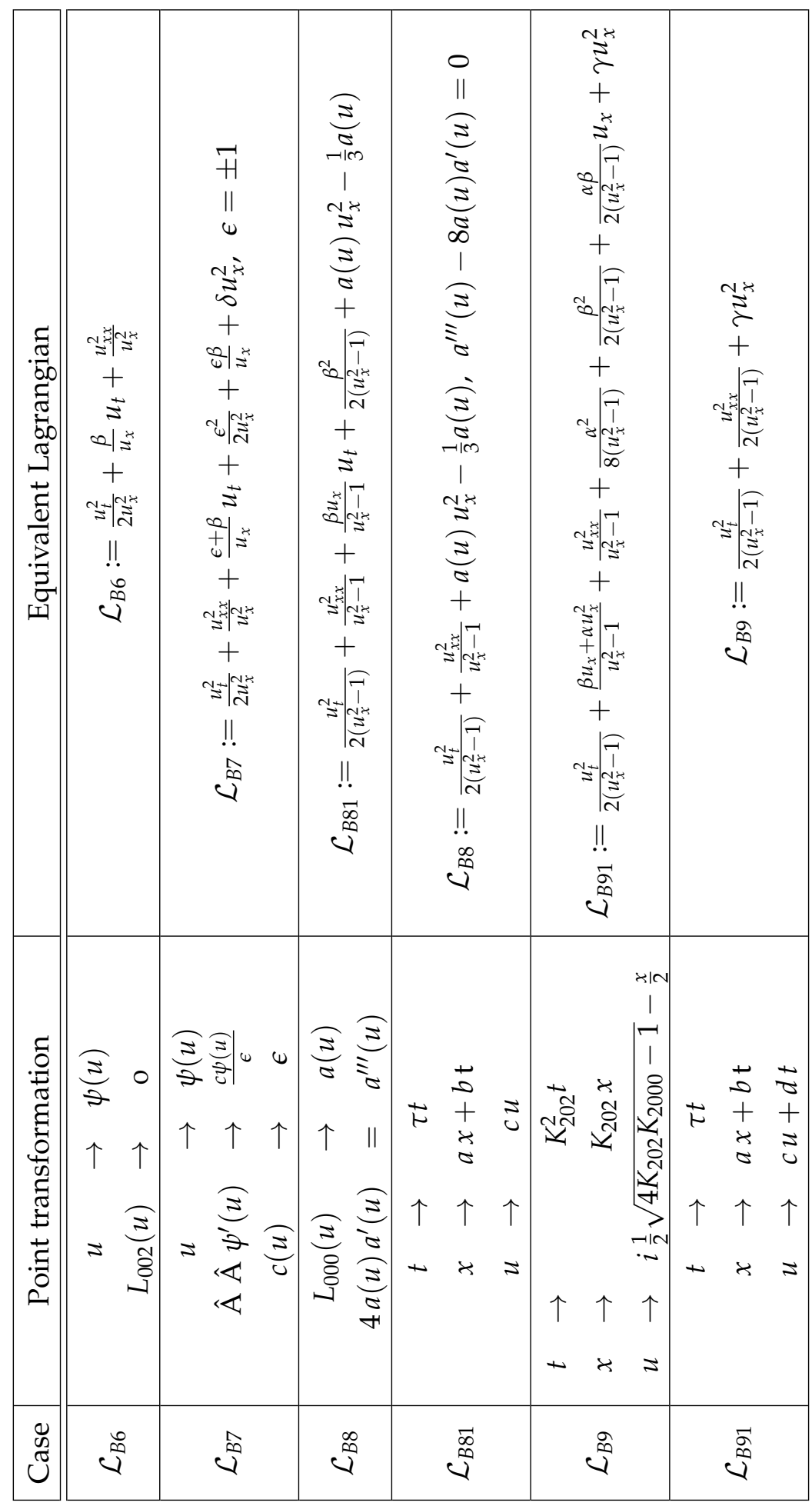

Table 5.4: Lagrangian densities after point transformations, 4 of 4 


\subsubsection{Equivalence between cases representatives}

There exist additional point transformations of the hodograph type revealing equivalences between cases. In table 5.5 we summarize such equivalences.

\subsection{LISTING OF NON-EQUIVALENT INTEGRABLE CASES}

Table 5.6 lists all the integrable cases found which are nonequivalent as obtained along the classification process detailed in this chapter.

A final relabeling attending to standard notation convention for constants and arbitrary functions leads to the final classes representatives listed in table 5.7. 


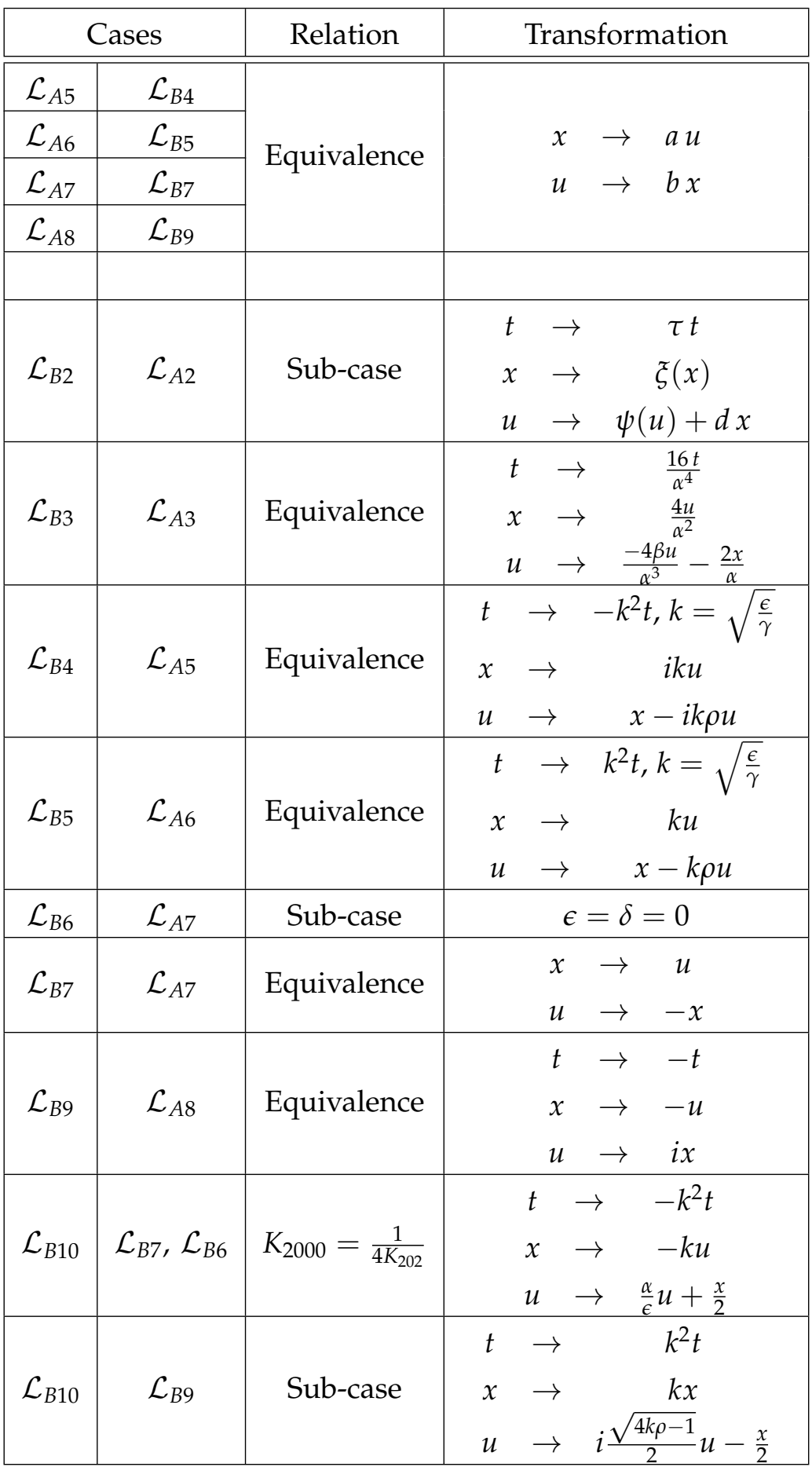

Table 5.5: Equivalence between case representatives 


\begin{tabular}{|c|}
\hline Final Integrable Cases \\
\hline \hline $\mathcal{L}_{B 1}:=\mathcal{L} 1=\frac{u_{t}^{2}}{2}+\epsilon u_{x} u_{t}+u_{x x}^{2}+\delta u_{x}^{2}+d(u)$ \\
\hline $\mathcal{L}_{B 3}:=\mathcal{L} 2=\frac{u_{t}^{2}}{2}+u_{x}^{2} u_{t}+u_{x x}^{2}+\frac{u_{x}^{4}}{2}+\delta_{2} u_{x}^{2}$ \\
\hline $\mathcal{L}_{A 4}:=\mathcal{L} 3=\frac{u_{t}^{2}}{2}+\frac{a(u)}{2} u_{t} u_{x}+\frac{u_{x x}^{2}}{u_{x}^{4}}+b(u) u_{x} \log u_{x}+\frac{a(u)^{2}}{8} u_{x}^{2}+c(u)$ \\
\hline $\mathcal{L}_{A 5}:=\mathcal{L} 4=\frac{u_{t}^{2}}{2}+\epsilon u_{x} u_{t}+\frac{u_{x x}^{2}}{u_{x}^{4}}+\delta_{2} u_{x} \log u_{x}+\frac{\epsilon^{2}}{2} u_{x}^{2}+\frac{\delta}{u_{x}}, \delta \neq 0$ \\
\hline $\mathcal{L}_{A 6}:=\mathcal{L} 5=\frac{u_{t}^{2}}{2}+\left(\frac{\gamma}{u_{x}}+\epsilon u_{x}\right) u_{t}+\frac{u_{x x}^{2}}{u_{x}^{4}}+\frac{\epsilon^{2}}{2} u_{x}^{2}+\frac{\gamma^{2}}{2 u_{x}^{2}}+\frac{\delta}{u_{x}}, \gamma \neq 0$ \\
\hline $\mathcal{L}_{A 7}:=\mathcal{L} 6=\frac{1}{2} u_{x} u_{t}^{2}+\left(\beta u_{x}+\epsilon u_{x}^{2}\right) u_{t}+\frac{u_{x x}^{2}}{u_{x}^{3}}+\frac{1}{2} \epsilon^{2} u_{x}^{3}+\epsilon \beta u_{x}^{2}+\frac{\gamma}{u_{x}}$ \\
\hline $\mathcal{L}_{A 1}:=\mathcal{L} 7=\frac{u_{t}^{2}}{2 u_{x}}+\frac{a(u)}{u_{x}} u_{t}+\frac{u_{x x}^{2}}{a(u)^{4} u_{x}^{5}}+\frac{b(u)}{u_{x}}+c(u)$ \\
\hline $\mathcal{L}_{A 2}:=\mathcal{L} 8=\frac{u_{t}^{2}}{2 u_{x}}+\epsilon \frac{u_{t}}{u_{x}}+\frac{u_{x x}^{2}}{u_{x}^{5}}+\frac{\delta}{u_{x}^{2}}+b(u), \delta \neq 0$ \\
\hline $\mathcal{L}_{B 8}:=\mathcal{L} 9=\frac{u_{t}^{2}}{2\left(u_{x}^{2}-1\right)}+\frac{u_{x x}^{2}}{2\left(u_{x}^{2}-1\right)}+\delta u_{x}^{2}$ \\
\hline $\mathcal{L}_{B 10}:=\mathcal{L} 10 \frac{u_{t}^{2}}{2\left(u_{x}^{2}-1\right)}+\frac{u_{x x}^{2}}{2\left(u_{x}^{2}-1\right)}+a(u) u_{x}^{2}-\frac{1}{3} a(u), \quad a^{\prime \prime \prime}(u)-8 a(u) a^{\prime}(u)=0$ \\
\hline
\end{tabular}

Table 5.6: Final integrable cases

\begin{tabular}{|c|c|}
\hline & Second order integrable Lagrangian systems class representatives \\
\hline \hline $\mathcal{L} 1$ & $\frac{u_{t}^{2}}{2}+\epsilon u_{x} u_{t}+u_{x x}^{2}+\alpha u_{x}^{2}+a(u)$ \\
\hline $\mathcal{L} 2$ & $\mathcal{L}_{2}=\frac{u_{t}^{2}}{2}+u_{x}^{2} u_{t}+u_{x x}^{2}+\frac{u_{x}^{4}}{2}+\alpha u_{x}^{2}$ \\
\hline $\mathcal{L} 3$ & $\frac{u_{t}^{2}}{2}+a(u) u_{t} u_{x}+\frac{u_{x x}^{2}}{u_{x}^{4}}+b(u) u_{x} \log u_{x}+\frac{a(u)^{2}}{8} u_{x}^{2}+c(u)$ \\
\hline $\mathcal{L} 4$ & $\frac{u_{t}^{2}}{2}+\epsilon u_{x} u_{t}+\frac{u_{x x}^{2}}{u_{x}^{4}}+\alpha u_{x} \log u_{x}+\frac{\epsilon^{2}}{2} u_{x}^{2}+\frac{\beta}{u_{x}}, \beta \neq 0$ \\
\hline $\mathcal{L} 5$ & $\frac{u_{t}^{2}}{2}+\left(\frac{\alpha}{u_{x}}+\epsilon u_{x}\right) u_{t}+\frac{u_{x x}^{2}}{u_{x}^{4}}+\frac{\epsilon^{2}}{2} u_{x}^{2}+\frac{\alpha^{2}}{2 u_{x}^{2}}+\frac{\beta}{u_{x}}, \alpha \neq 0$ \\
\hline $\mathcal{L} 6$ & $\frac{1}{2} u_{x} u_{t}^{2}+\left(\alpha u_{x}+\epsilon u_{x}^{2}\right) u_{t}+\frac{u_{x x}^{2}}{u_{x}^{3}}+\frac{1}{2} \epsilon^{2} u_{x}^{3}+\epsilon \alpha u_{x}^{2}+\frac{\beta}{u_{x}}$ \\
\hline $\mathcal{L} 7$ & $\frac{u_{t}^{2}}{2 u_{x}}+\frac{a(u)}{u_{x}} u_{t}+\frac{u_{x x}^{2}}{a(u)^{4} u_{x}^{5}}+\frac{b(u)}{u_{x}}+c(u)$ \\
\hline $\mathcal{L} 8$ & $\frac{u_{t}^{2}}{2 u_{x}}+\epsilon \frac{u_{t}}{u_{x}}+\frac{u_{x x}^{2}}{u_{x}^{5}}+\frac{\alpha}{u_{x}^{2}}+\frac{a(u)}{u_{x}}+b(u), \alpha \neq 0$ \\
\hline $\mathcal{L} 9$ & $\frac{u_{t}^{2}}{2\left(u_{x}^{2}-1\right)}+\frac{u_{x x}^{2}}{2\left(u_{x}^{2}-1\right)}+\gamma u_{x}^{2}$ \\
\hline $\mathcal{L} 10$ & $\frac{u_{t}^{2}}{2\left(u_{x}^{2}-1\right)}+\frac{u_{x x}^{2}}{2\left(u_{x}^{2}-1\right)}+a(u) u_{x}^{2}-\frac{1}{3} a(u), \quad a^{\prime \prime \prime}(u)-8 a(u) a^{\prime}(u)=0$ \\
\hline
\end{tabular}

Table 5.7: Second order integrable Lagrangian systems class representatives (up to $\rho_{6}$ ) 


\subsection{ADDITIONAL CONSIDERATIONS}

The following considerations regarding the classification process and results apply.

- The classification results listed in 5.7 are as obtained "up to $\rho_{6}{ }^{\prime \prime}$. There were seven canonical densities utilized $\left\{\rho_{0}, \cdots, \rho_{6}\right\}$ as listed in table 2.1. This means that the functional form of the integrable cases can be further specified, in some cases, if more canonical densities were given as input to the ILSC procedure. Although this is theoretically computable, the size of the expanded expressions for higher densities (which can be checked by inspecting $\rho_{7}$ in the appendix) makes the problem's computational constrains very strong.

- Integrability of all integrable cases was checked by reversing the CCL integration procedure for each one of them. These processes successfully result in a number of explicit conservation laws (as many as conserved densities) which can theoretically grow unbounded, therefore proving each case's integrability. These computations are described in section 4.4

- In some of the integrable cases found (i.e: $\mathcal{L}_{2}$ of the NLS type in example 21) the utilization of additional canonical densities has no use because all the obstructions found were integrated and there are no more obstructions.

- In some particular cases the classification process can be extended to $\rho_{7}, \rho_{8}, \rho_{9}, \rho_{10}$ resulting in additional integrability obstructions for each of the following cases:

- $\mathcal{L} 1$ : it must be $a^{\prime \prime \prime}(u)=0$ so this Lagrangian is purely quadratic with linear Euler-Lagrange equation.

- $\mathcal{L} 2$ : no more obstructions found, additional point transformations allow to set $\alpha=0$ resulting in an NLS-type Lagrangian.

- $\mathcal{L} 3$ : it must be $b(u)=\sqrt{2} a^{\prime}(u)^{2}$ with no more obstructions found.

- $\mathcal{L} 4$ : it is $\alpha=0$ this case merges with with $\mathcal{L}_{5}$ allowing $\beta=0$.

- $\mathcal{L} 8$ : additional obstruction $\alpha\left[-2 a^{\prime}(u)+3 \alpha b(u)+2 a^{\prime \prime \prime}(u)\right]=$ 0 opens two sub-cases

$* \alpha=0$ is a sub-case of $\mathcal{L}_{7}$,

* $\alpha \neq 0$ implies $b(u)=\frac{1}{3 \alpha}\left[a(u)^{2}-2 b^{\prime \prime}(u)\right]=0$. Boussinesq Equation. 
- Table 5.7 lists the class representatives without the considerations in this section. The following table 5.8 lists an alternative list of functional expressions for ILSC taking in to account the considerations in this section and choosing representatives that appear more frequently in the literature. These expressions are calculated as in section 5.5 by finding allowed transformations (chapter 3).

Euler-Lagrange equations for each case are listed below

- $\mathcal{L} 1$ LINEAR CASE

$$
u_{t t}=\alpha u_{x x x x}-2 \epsilon u_{x t}-\beta u_{x x}+\gamma
$$

- $\mathcal{L} 2$ NLS-type

$$
u_{t t}=u_{x x x x}-3 u_{x x} u_{x}
$$

- $\mathcal{L} 3$ BOUSSINESQ

$$
u_{t t}=u_{x x x x}+4 u_{x t} u_{x}+2 u_{x x} u_{t}-6 u_{x}^{2} u_{x x}
$$

- $\mathcal{L} 4$

$$
\begin{aligned}
u_{t t} & =\frac{1}{u_{x}^{6}}\left(-a(u)^{2} u_{x}^{6} u_{x x}+10 u_{x x}^{3}-2 a(u) u_{x}^{6} u_{x t}-8 u_{x x x} u_{x x} u_{x}+u_{x}^{2} u_{x x x x}\right) \\
& +\frac{1}{u_{x}^{6}}\left(-a(u) a^{\prime}(u) u_{x}^{8}-a^{\prime}(u) u_{x}^{7} u_{t}-a^{\prime}(u) u_{x}^{5} u_{x x}+b^{\prime}(u) u_{x}^{6}-a^{\prime \prime}(u) u_{x}^{7}\right)
\end{aligned}
$$

- $\mathcal{L} 5$

$$
\begin{aligned}
u_{t t} & =\frac{1}{u_{x}^{6}}\left(-3 \alpha^{2} u_{x}^{2} u_{x x}-2 \beta u_{x}^{3} u_{x x}-\epsilon^{2} u_{x}^{6} u_{x x}-2 \alpha u_{x}^{3} u_{x x} u_{t}\right) \\
& +\frac{1}{u_{x}^{6}}\left(10 u_{x x}^{8}+2 \alpha u_{x}^{4} u_{x t}-2 \epsilon u_{x}^{6} u_{x t}-8 u_{x x x} u_{x x} u_{x}+u_{x}^{2} u_{x x x x}\right)
\end{aligned}
$$

- $\mathcal{L} 6$

$$
\begin{aligned}
u_{t t} & =\frac{1}{u_{x}^{6}}\left(-2 \beta u_{x}^{2} u_{x x}-2 \alpha \epsilon u_{x}^{5} u_{x x}-3 \epsilon^{2} u_{x}^{6} u_{x x}-2 \epsilon u_{x}^{5} u_{x x} u_{t}+6 u_{x x}^{3}\right) \\
& +\frac{1}{u_{x}^{6}}\left(-2 \alpha u_{x}^{5} u_{x t}-4 \epsilon u_{x}^{6} u_{x t}-2 u_{x}^{5} u_{x t} u_{t}-6 u_{x x x} u_{x x} u_{x}+u_{x}^{2} u_{x x x x}\right)
\end{aligned}
$$

- $\mathcal{L} 7$

$$
\begin{aligned}
u_{t t} & =\frac{-1}{a(u)^{6} u_{x}^{6}}\left(2 a(u)^{6} b(u) u_{x}^{4} u_{x x}+2 a(u)^{7} u_{x}^{4} u_{x x} u_{t}+a(u)^{6} u_{x}^{4} u_{t}^{2} u_{x x}-30 a(u)^{2} u_{x x}^{3}\right) \\
& +\frac{1}{a(u)^{6} u_{x}^{6}}\left(-2 a(u)^{7} u_{x}^{5} u_{x t}-2 a(u)^{6} u_{x}^{5} u_{x t} u_{t}+20 a(u)^{2} u_{x x x} u_{x x} u_{x}-2 a(u)^{2} u_{x}^{2} u_{x x x x}\right) \\
& +\frac{1}{a(u)^{6} u_{x}^{6}}\left(-a(u)^{6} a^{\prime}(u) u_{x}^{6} u_{t}-48 a(u) a^{\prime}(u) u_{x}^{2} u_{x x}^{2}+16 a(u) a^{\prime}(u) u_{x}^{3} u_{x x x}\right) \\
& +\frac{1}{a(u)^{6} u_{x}^{6}}\left(-40 a^{\prime}(u)^{2} u_{x}^{4} u_{x x}-2 a(u)^{6} b^{\prime}(u) u_{x}^{6}+8 a(u) a^{\prime \prime}(u) u_{x}^{4} u_{x x}\right)
\end{aligned}
$$


- $\mathcal{L} 8$

$$
\begin{aligned}
u_{t t} & =\frac{-1}{\left(u_{x}+1\right)^{2}\left(u_{x}-1\right)^{2}}\left(-2 a(u)+6 a(u) u_{x}^{2}-6 a(u) u_{x}^{4}\right) \\
& +\left(2 a(u) u_{x}^{6}+u_{t}^{2}+3 u_{x}^{2} u_{t}^{2}\right) u_{x x} \\
& +\frac{-1}{\left(u_{x}+1\right)^{2}\left(u_{x}-1\right)^{2}}\left(1+3 u_{x}^{2}\right) u_{x x}^{3}+\frac{4 u_{x t} u_{x} u_{t}}{\left(u_{x}+1\right)\left(u_{x}-1\right)} \\
& +u_{x x x x}+\frac{1}{3}\left(1+3 u_{x}^{2}\right) a^{\prime}(u)
\end{aligned}
$$

For most of the integrable cases found in the it has been possible to find a recursion operator in closed form, thus providing sufficient conditions of their integrability. The construction method for these is described in chapter 6. 


\section{Second order integrable Lagrangian systems class representatives}

\begin{tabular}{|c|c|}
\hline $\mathcal{L} 1$ & $\begin{array}{c}\text { LINEAR } \\
\frac{u_{t}^{2}}{2}+\epsilon u_{x} u_{t}+\alpha \frac{u_{x x}^{2}}{2}+\beta \frac{u_{x}^{2}}{2}+\gamma u\end{array}$ \\
\hline $\mathcal{L} 2$ & $\begin{array}{c}\text { NLS-type } \\
\frac{1}{2}\left(u_{t}-u_{x}^{2}\right)^{2}+\frac{u_{x x}^{2}}{2}\end{array}$ \\
\hline $\mathcal{L} 3$ & $\begin{array}{c}\text { BOUSSINESQ } \\
\frac{1}{2}\left(u_{t}+u_{x x}\right)^{2}+\frac{u_{x}^{3}}{2}\end{array}$ \\
\hline $\mathcal{L} 4$ & $\frac{u_{t}^{2}}{2}+a(u) u_{t} u_{x}+\frac{u_{x x}^{2}}{2 u_{x}^{4}}+a^{\prime}(u) u_{x} \log u_{x}+\frac{a(u)^{2}}{2} u_{x}^{2}+b(u)$ \\
\hline $\mathcal{L} 5$ & $\begin{array}{c}\frac{u_{t}^{2}}{2}+\left(\frac{\alpha}{u_{x}}+\epsilon u_{x}\right) u_{t}+\frac{u_{x x}^{2}}{2 u_{x}^{4}}+\frac{\epsilon^{2}}{2} u_{x}^{2}+\frac{\alpha^{2}}{2 u_{x}^{2}}+\frac{\beta}{u_{x}} \\
|\alpha|+|\beta| \neq 0\end{array}$ \\
\hline $\mathcal{L} 6$ & $\begin{array}{c}\frac{1}{2} u_{x} u_{t}^{2}+\left(\alpha u_{x}+\epsilon u_{x}^{2}\right) u_{t}+\frac{u_{x x}^{2}}{2 u_{x}^{3}}+\frac{1}{2} \epsilon^{2} u_{x}^{3}+\epsilon \alpha u_{x}^{2}+\frac{\beta}{u_{x}} \\
\alpha \neq 0\end{array}$ \\
\hline $\mathcal{L} 7$ & $\frac{u_{t}^{2}}{2 u_{x}}+\frac{a(u)}{u_{x}} u_{t}+\frac{u_{x x}^{2}}{a(u)^{4} u_{x}^{5}}+\frac{b(u)}{u_{x}}$ \\
\hline $\mathcal{L} 8$ & $\begin{array}{c}\frac{u_{t}^{2}}{2\left(u_{x}^{2}-1\right)}+\frac{u_{x x}^{2}}{2\left(u_{x}^{2}-1\right)}+a(u) u_{x}^{2}-\frac{1}{3} a(u) \\
a^{\prime \prime \prime}(u)-8 a(u) a^{\prime}(u)=0\end{array}$ \\
\hline
\end{tabular}

Table 5.8: Second order integrable Lagrangian systems class representatives (up to $\rho_{10}$ ) 

Finding recursion operators is a central problem of the theory of integrable systems which links the two generic types of integrability mentioned in sections 1.5 and 1.6. Constructability of solutions and spectral methods like the inverse scattering transform agree when equations can be written in operator form. Like finding Lax pairs, the construction of recursion operators is only completely determined for some well known equations. Reader can check Sanders and Wang [42] for the structure of recursion operators of evolution equations. These have generally a local and a nonlocal part which is determined by symmetries and cosymmetries, enabling their computation in a systematic way ${ }^{1}$.

In this chapter we explain how recursion operators can be derived from the symmetry condition when applied to non evolutionary equations of the families studied in this thesis and whose integrability is understood under the extended symmetry approach of chapter 2 . Like in the evolutionary case (section 1.8), the algebraic structure of the space of solutions of the linearized symmetry condition holds interesting properties that encode the equation's integrability. Exploiting those properties allowed us to classify higher order integrable Lagrangian systems in chapter 5. Furthermore, the properties of the space of formal recursion operators for such equations allows us to derive the general structure of the space of symmetries starting from the determining equations of formal recursion operators, as described in the next section. Finally, closed form operators are found for the integrable cases resulting of the classification, thus giving sufficient conditions of their integrability.

6.1 DETERMINING EQUATIONS FOR FORMAL RECURSION OPERATORS OF NON-EVOLUTIONARY EQUATIONS.

Following the problem introduced in section 2.4 and with the notations in section 1.14, for symmetries

$$
u_{\tau}=G[u]=G\left(u, u_{x}, \ldots, u_{m} ; u_{t}, u_{x t}, \ldots u_{n t}\right)
$$

of order $(4,1)$ non evolutionary equations

$$
\begin{aligned}
H & =u_{t t}-F[u] \\
& =u_{t t}-F\left(u, u_{x}, \ldots, u_{4} ; u_{t}, u_{x t}\right)
\end{aligned}
$$

1 We will employ an ansatz for this in section 6.2.

Closed form recursion operators differ from formal recursion operators in being

"differential": including only positive powers of derivatives, and a finite number of terms, as oposed to pseudo-differential infinite series defining formal recursion operators. 
the symmetry condition is

$$
D_{t}^{2} \doteq F^{*} G
$$

we aim to find the symmetry definition condition in operators form.

For the Frechet linearization of the two equations above

$$
\begin{aligned}
& \phi_{\tau}=G^{*} \phi \\
& \phi_{t t}=F^{*} \phi
\end{aligned}
$$

the compatibility condition is $\phi_{t t \tau}=\phi_{\tau t}$, corresponding to $F_{\tau}=$ $u_{t t \tau}, G_{t}=u_{\tau t}$, which can be expanded as

$$
\left(F^{*} \phi\right)_{\tau}-\left(G^{*} \phi\right)_{t t}=0
$$

giving

$$
\begin{aligned}
\phi_{t t \tau}-\phi_{\tau t} & =\left(F^{*}\right)_{\tau} \phi+F^{*} \phi_{\tau}-\left(\left(G^{*}\right)_{t} \phi+G^{*} \phi_{t}\right)_{t} \\
& =F_{\tau}^{*} \phi+F^{*} G^{*} \phi-\left(G_{t}^{*} \phi+G^{*} \phi_{t}\right)_{t} \\
& =F_{\tau}^{*} \phi+F^{*} G^{*} \phi \\
& -G_{t t}^{*} \phi-G_{t}^{*} \phi_{t}-G_{t}^{*} \phi_{t}-G_{t}^{*} \phi_{t t} \\
& =F_{\tau}^{*} \phi+F^{*} G^{*} \phi-G_{t t}^{*} \phi-2 G_{t}^{*} \phi_{t}-G_{t}^{*} \phi_{t t}
\end{aligned}
$$

from where

$$
\begin{aligned}
F_{\tau}^{*} \phi & =G_{t t}^{*} \phi+2 G_{t}^{*} \phi_{t}+G_{t}^{*} \phi_{t t}-F^{*} G^{*} \phi \\
& =G_{t t}^{*} \phi+2 G_{t}^{*} \phi_{t}+G_{t}^{*} F^{*} \phi-F^{*} G^{*} \phi
\end{aligned}
$$

which can be expressed in operator form by using vector notation

$$
\Phi=\left(\begin{array}{c}
\phi \\
\phi_{t}
\end{array}\right), \quad \Phi_{t}=\left(\begin{array}{c}
\phi_{t} \\
\phi_{t t}
\end{array}\right)=\left(\begin{array}{c}
\phi_{t} \\
F^{*} \phi
\end{array}\right)
$$

and with

$$
\begin{aligned}
\mathcal{G} & =G^{*} \\
& =G_{u}+\ldots+G_{u_{m}} D_{x}^{m}+G_{u_{t}} D_{x} D_{t}+\ldots+G_{u_{n t}} D_{x}^{n} D_{t} \\
& =L+M D_{t} \\
\mathcal{F} & =F^{*} \\
& =F_{u}+F_{u_{x}} D_{x}+\ldots+F_{u_{4}} D_{x}^{4}+F_{u_{t}} D_{x} D_{t}+F_{u_{x t}} D_{x} D_{t} \\
& =A+B D_{t}
\end{aligned}
$$

resulting in ${ }^{2}$

$$
\Phi_{t}=\left(\begin{array}{ll}
0 & 1 \\
A & B
\end{array}\right) \Phi=\mathbf{F} \Phi
$$

2 We will use bold symbols denoting generic matrix differential operators 
which allows an expression of the symmetry

$$
\begin{aligned}
\Phi_{\tau}=\left(\begin{array}{c}
\phi_{\tau} \\
\phi_{\tau t}
\end{array}\right) & =\left(\begin{array}{c}
G^{*} \phi \\
G_{t}^{*} \phi
\end{array}\right)=\left(\begin{array}{c}
\mathcal{G} \phi \\
(\mathcal{G} \phi)_{t}
\end{array}\right) \\
& =\left(\begin{array}{c}
\left(L+M D_{t}\right) \phi \\
\left(\left(L+M D_{t}\right) \phi\right)_{t}
\end{array}\right) \\
& =\left(\begin{array}{c}
L \phi+M \phi_{t} \\
\left(L \phi+M \phi_{t}\right)_{t}
\end{array}\right) \\
& =\left(\begin{array}{c}
\left(M_{t}+M B\right) \phi+\left(M_{t}+L+M A\right) \phi_{t}
\end{array}\right) \\
& =\left(\begin{array}{c}
L \\
L_{t}+M B
\end{array}\right)\left(\begin{array}{c}
\phi \\
\phi_{t}
\end{array}\right) \\
& =\left(\begin{array}{cc}
L & M \\
\widehat{L} & \widehat{M}
\end{array}\right)\left(\begin{array}{c}
\phi \\
\phi_{t}
\end{array}\right) \\
& =\mathbf{G} \Phi
\end{aligned}
$$

As in the evolutionary case, this matrix equation can be linearized and for an arbitrarily high order symmetry $G$

$$
\mathbf{F}_{\tau}=[\mathbf{F}, \mathbf{G}]-\mathbf{G}_{t}
$$

from where we obtain the recursion operator components as

$$
\begin{aligned}
& \mathbf{R}_{t}=\left(\begin{array}{cc}
L_{t} & M_{t} \\
\widehat{L}_{t} & \widehat{M}_{t}
\end{array}\right)=[\mathbf{F}, \mathbf{G}] \\
& =\left(\begin{array}{ll}
0 & 1 \\
A & B
\end{array}\right)\left(\begin{array}{ll}
L & M \\
\widehat{L} & \widehat{M}
\end{array}\right)-\left(\begin{array}{ll}
L & M \\
\widehat{L} & \widehat{M}
\end{array}\right)\left(\begin{array}{ll}
0 & 1 \\
A & B
\end{array}\right)= \\
& =\left(\begin{array}{cc}
\widehat{L} & \widehat{M} \\
A L+B \widehat{L} & A M+B \widehat{M}
\end{array}\right)-\left(\begin{array}{cc}
M A & L+M B \\
\widehat{M} A & \widehat{L}+\widehat{M} B
\end{array}\right) \\
& =\left(\begin{array}{cc}
\widehat{L}-M A & \widehat{M}-L-M B \\
A L+B \widehat{L}-\widehat{M} A & A M+B \widehat{M}-\widehat{L}-\widehat{M} B
\end{array}\right) \\
& L_{t}=\widehat{L}-M A \\
& M_{t}=\widehat{M}-L-M B \\
& \widehat{L}_{t}=A L+B \widehat{L}-\widehat{M} A \\
& \widehat{M}_{t}=A M+B \widehat{M}-\widehat{L}-\widehat{M} B
\end{aligned}
$$

which combined result in

$$
\begin{aligned}
\widehat{L}_{t} & =A L+B \widehat{L}-\widehat{M} A=\left(L_{t}+M A\right)_{t}=L_{t t}+M_{t} A+M A_{t} \\
\widehat{M}_{t} & =A M+B \widehat{M}-\widehat{L}-\widehat{M} B=\left(M_{t}+L+M B\right)_{t}=M_{t t}+L_{t}+M_{t} B+M B_{t}
\end{aligned}
$$


To get to these equations, we have written the symmetry conditions in matrix notation.

Equivalently (and more efficiently for computations) scalar notation can be used (see 2.3).

$$
\begin{array}{r}
L_{t t}+M_{t} A+M A_{t}-A L-B\left(L_{t}+M A\right)+\left(M_{t}+L+M B\right) A=0 \\
M_{t t}+L_{t}+M_{t} B+M B_{t}-A M-B\left(M_{t}+L+M B\right)+L_{t}+M A+\left(M_{t}+L+M B\right) B=0
\end{array}
$$

and can be expanded taking into account non-commutativity of differential operators product as

$$
\begin{aligned}
L_{t t}+M_{t} A+M A_{t}-A L-B L_{t}-B M A+M_{t} A+L A+M B A & =0 \\
M_{t t}+L_{t}+M_{t} B+M B_{t}-A M-B M_{t}-B L-B M B+L_{t}+M A+M_{t} B+L B+M B B & =0
\end{aligned}
$$

from where the symmetry conditions yield the recursion operator components defining equations

$$
\begin{array}{r}
L_{t t}-B L_{t}+[L, A]+\left(2 M_{t}+[M, B]\right) A+M A_{t}=0 \\
M_{t t}+2 L_{t}+[M, A]+[L, B]+\left(2 M_{t}+[M, B]\right) B+M B_{t}-B M_{t}=0
\end{array}
$$

In the determining equations obtained above we have to keep in mind that, on the contrary to the procedure utilized in the ILSC.

- $A, B$ are given expressions depending on the equation $H=0$ or $u_{t t}=F[u]$ for each of the integrable cases listed in table 5.8.

- $L, M$ and have been defined as

$$
\begin{aligned}
L & :=G_{u}+\ldots+G_{u_{m}} D_{x}^{m} \\
M & :=G_{u_{t}} D_{x}+\ldots+G_{u_{n t}} D_{x}^{n}
\end{aligned}
$$

and are unknowns to be calculated after integration of the conditions 6.1.4 which consists of the system of PDEs obtained equating every coefficient of $D^{j}$ in the 1.h.s's to the corresponding coefficient in the r.h.s's.

- They can be used to find symmetries of a fixed differential order $(r, s)$ of 6.1.3. For such symmetries with $r \gg n, s \gg m$, the number of conditions to be met in the left hand sides grows with $r$ and $s$ and following the symmetry approach method, they can grow unbounded. For this reason they are taken as pseudodifferential operators, formal series or formal recursion operators. 


\subsection{RECURSION OPERATORS OF INTEGRABLE LAGRANGIAN SYS- TEMS}

In this section all the classes given in table 5.8 are proven to be integrable except for $\mathcal{L}_{4}$ and $\mathcal{L}_{7}$, that presented computational problems that are beyond the capability of our computing facilities. For these two classes, nevertheless, we give examples that probably exhaust their integrable cases. From now on, when we say that a Lagrangian or a system admits a symmetry or recursion operator, we are of course referring to the corresponding Euler-Lagrange equations listed in section 5.7 .

The proof of integrability is achieved by giving an explicit, nonformal recursion operator in closed form and corresponding seed symmetries. The recursion operator can be found by inspection in a few cases, but usually this is quite difficult to do. The general approach that worked here is to use the ansatz in Demskoi and Sokolov [9] and Sokolov [44] for a recursion operator of the form

$$
\mathcal{R}=\mathcal{D}+\sum_{k} s_{k} D^{-1} \cdot \mathcal{C}_{k}
$$

being

- $\mathcal{D}$ a differential operator, as the first or local part of the operator

- the sum (non local part, with inverse derivatives) a linear combination where

- the coefficients $s_{k}$ are symmetries

$-\mathcal{C}_{k}$ are linearisations of conserved densities $\rho_{k}$.

\subsubsection{Linear Lagrangian $\mathcal{L} 1$}

This is the linear system, therefore

- it admits as recursion operator any linear combination with constant coefficients of the operators $D_{x}$ and $D_{t}$,

- the seed symmetry is $u$, and every derivative of the field is a symmetry.

\subsubsection{Lagrangian $\mathcal{L} 2$}

This system is related to the NLS equation listed in section 2.4 and simple inspection shows that $\mathcal{M}_{-1}$ (as defined in 2.3) is a recursion operator:

$$
\mathcal{R}_{2}=\mathcal{M}_{-1}=-u_{x}+2 D^{-1} \cdot u_{x x}+D^{-1} D_{t}
$$

This operator applied to the symmetry 1 produces $u_{x}$, and applied again to the latter produces $u_{t}$. Iterated, $\mathcal{R}$ generates a basis of the 
whole symmetry hierarchy. The first higher symmetries in this case are

$$
\begin{aligned}
& s_{1}^{a}:=u_{x x x}+3 u_{x} u_{t}-2 u_{x}^{3}, \\
& s_{1}^{b}:=u_{x x t}+2 u_{x} u_{x x x}-\frac{1}{2} u_{x x}^{2}+\frac{3}{2} u_{t}^{2}+3 u_{x}^{2} u_{t}-\frac{7}{2} u_{x}^{4} .
\end{aligned}
$$

System $\mathcal{L}_{2}$ admits a symmetry algebra with a definite structure. We will refer to this symmetry structure as a standard symmetry structure for non-evolutionary equations

$$
u_{t t}=f\left(u, u_{x}, \ldots, u_{n} ; u_{t}, u_{x t}, \ldots, u_{m t}\right)
$$

The standard structure consists of an infinite sequence of symmetries of two types. We will use the following notation to denote symmetries with different types of higher order terms (the ellipsis indicates lower order terms):

- One type is composed of symmetries with highest order term $c_{i} u_{i}$ where $i$ is an odd positive integer,

symmetries of type a: $s_{i}^{\mathrm{a}}=c_{i} u_{2 i+1}+\cdots, \quad i=0,1, \ldots$

- and the other type with higher order term $d_{j} u_{j t}$, where $j$ is an even positive integer.

$$
\text { symmetries of type b: } s_{j}^{\mathrm{b}}=d_{j} u_{2 j t}+\cdots, \quad j=0,1, \ldots
$$

The recursion operator acts over symmetries as

$$
\mathcal{R}_{2}\left(s_{i}^{a}\right)=s_{i}^{b} \text { and } \mathcal{R}_{2}\left(s_{j}^{b}\right)=s_{j+1}^{a}, i, j=0,1, \ldots
$$

Below we will show some systems with symmetry algebras being a variation to this general structure.

\subsubsection{Lagrangian $\mathcal{L} 3$}

This system is related to the potential Boussinesq equation and it admits the recursion operator $\mathcal{M}_{1}$ (as defined in 2.3):

$$
\mathcal{R}_{3}=\mathcal{M}_{1}=-\frac{9}{8} u_{t}+\frac{3}{8} D^{-1} \cdot u_{x t}+\left[D-\frac{3}{8} u_{x} D^{-1}-\frac{3}{8} D^{-1} \cdot u_{x}\right] D_{t} .
$$

Applied to $-4 / 3$ it yields $u_{t}$, applied to $u_{x}$ it gives $s_{1}^{b}=u_{x x t}-3 u_{x} u_{t} / 2$, applied to this symmetry yields a symmetry $s_{3}^{a}=u_{7}+\cdots$, applied to $u_{t}$, a symmetry $s_{2}^{a}=u_{5}+\cdots$, applied to the latter gives $s_{3}^{b}=$ $u_{6 t}+\cdots$ and so on. In general, $\mathcal{R}_{3}\left(s_{i}^{\mathrm{a}}\right)=s_{i+1}^{\mathrm{b}}$ and $\mathcal{R}_{3}\left(s_{j}^{\mathrm{b}}\right)=s_{j+2}^{\mathrm{a}}$, $i, j=0,1, \ldots$. Iterated, $\mathcal{R}_{3}$ generates a basis of the whole symmetry hierarchy that differs from the standard structure explained for $\mathcal{L}_{2}$ because it lacks all symmetries of the form $s_{1+3 i}^{\mathrm{a}}$ and $s_{2+3 j}^{\mathrm{b}}$ with $i, j=$ $0,1, \ldots$. This "defect" is probably due to the fact that the potential 
Boussinesq system is related to the usually called Boussinesq equation through a potentiation, a nonlocal transformation. The Boussinesq equation has more symmetries than the potential Boussinesq equation, and some symmetries of the former could become nonlocal for the latter. But we are dealing here only with equations admitting local symmetries and local recursion operators.

\subsubsection{Lagrangian $\mathcal{L} 3$ (matrix form)}

As an example following the notations introduced in this chapter for the derivation of the determining equation of recursion operators in matrix form, we derive a matrix recursion operator for one of the standard cases found

$$
\mathcal{L}_{3}=\frac{1}{2}\left(u_{t}+u_{x x}\right)^{2}+\frac{u_{x}^{3}}{2} \quad(\text { BOUSSINESQ })
$$

for which the corresponding Euler-Lagrange equation (listed in section 5.7)

$$
u_{t t}=u_{x x x x}+4 u_{x} u_{x t}+2 u_{x x} u_{t}-6 u_{x}^{2} u_{x x}
$$

we have

$$
\begin{aligned}
A & =D^{4}+\left(2 u_{t}-6 u_{x}^{2}\right) D^{2}+\left(4 u_{x t}-12 u_{x} u_{x x}\right) D \\
B & =4 u_{x} D+2 u_{x x}
\end{aligned}
$$

resulting in

$$
\mathbf{R}_{3}=\left(\begin{array}{cc}
-2 u_{x} D+2 D^{-1} u_{x x} & D^{-1} \\
D^{3}+2\left(u_{t}-3 u_{x}^{2}\right) D+u_{x t} & 3 u_{x}
\end{array}\right)
$$

\subsubsection{Lagrangian $\mathcal{L} 4$}

This case comprises a big family of systems depending on two arbitrary functions $a(u), b(u)$. Surprisingly, this family satisfies many integrability conditions, and with our present computing resources (and algorithms) we cannot restrict the class further. The generic case, with $a^{\prime}(u) \neq 0$, does not admit higher symmetries of differential order up to five, so it seems nonintegrable. Most probably, the obstruction to integrability $c_{i}$ that restricts the family is of very high level $i \gg 10$.

In order to illustrate this case we suppose that $a(u)=\alpha$ is constant and show how in this case the system is indeed integrable. With $a(u)=$ constant $=\alpha$, system $\mathcal{L}_{4}$ posseses the conserved densities

$$
\begin{aligned}
\rho_{1} & =u_{t} u_{x}+\alpha u_{x}^{2} \\
\rho_{2} & =\frac{u_{x x}^{2}}{2 u_{x}^{4}}-\frac{u_{t}^{2}}{2}+\frac{\alpha^{2} u_{x}^{2}}{2}+d(u)
\end{aligned}
$$


with associated operators

$$
\begin{gathered}
\mathcal{C}_{1}=\left(2 \alpha u_{x}+u_{t}\right) D+u_{x} D_{t} \\
\mathcal{C}_{2}=\frac{u_{x x}}{u_{x}^{4}} D^{2}+\left(\alpha^{2} u_{x}-2 \frac{u_{x x}^{2}}{u_{x}^{5}}\right) D+d^{\prime}(u)-u_{t} D_{t}
\end{gathered}
$$

and a recursion operator

$$
\begin{aligned}
\mathcal{R}_{41} & =\mathcal{L}_{2}=\frac{1}{u_{x}^{2}} D^{2}-\frac{5}{2} \frac{u_{x x}}{u_{x}^{3}} D-\frac{u_{x x x}}{u_{x}^{3}}+\frac{9}{4} \frac{u_{x x}^{2}}{u_{x}^{4}}+ \\
& +\frac{3}{4} u_{t}^{2}+\frac{3}{2} \alpha u_{x} u_{t}+\frac{3}{4} \alpha^{2} u_{x}^{2}-\frac{1}{2} d(u) \\
& -\left(\alpha u_{x}+\frac{1}{2} u_{t}\right) D^{-1} \mathcal{C}_{1}+\frac{1}{2} u_{x} D^{-1} \mathcal{C}_{2}
\end{aligned}
$$

For a generic $d(u)$ this equation has only higher symmetries of type $s_{j}^{b}$, $j \geq 1$ generated by $\mathcal{R}_{41}$ with a seed symmetry $u_{t}$, i.e. $\mathcal{R}_{41}\left(s_{j}^{b}\right)=s_{j+1}^{b}$, $j \geq 0$. There are interesting subcases admitting more symmetries, depending on the form of $d(u)$.

- The subcase with $d^{\prime \prime}(q)=3 d^{2}(q) / 2$. This system additionally admits all generalised symmetries of the form $s_{i}^{a}$, and more conservation laws. The recursion operator $\mathcal{R}_{41}=\mathcal{L}_{2}$ is able to generate a standard symmetry structure starting with seed symmetry $s_{0}^{b}=u_{t}$, and a second seed symmetry

$$
s_{1}^{a}=\frac{u_{x x x}}{u_{x}^{3}}-\frac{9 u_{x x}^{2}}{4 u_{x}^{4}}-\frac{3 u_{t}^{2}}{4}-\frac{3}{2} \alpha u_{x} u_{t}-\frac{3}{4} \alpha^{2} u_{x}^{2}+\frac{4}{3} d(u)
$$

with $\mathcal{R}_{41}\left(s_{i}^{a}\right)=s_{i+1}^{a}, i \geq 1$. There exists an additional independent recursion operator $\mathcal{R}_{42}=\mathcal{M}_{1}$ that can be written in finite form as

$$
\begin{aligned}
\mathcal{R}_{42} & =\mathcal{M}_{1}=-\frac{1}{2} \frac{3 u_{t}+\alpha u_{x}}{u_{x}^{2}} D^{2}-\left(\frac{3}{2} \frac{u_{x t}}{u_{x}^{2}}-\frac{5}{4} \frac{3 u_{t}+\alpha u_{x}}{u_{x}^{3}} u_{x x}\right) D-s_{1}^{b} \\
& -\frac{1}{8}\left(2 u_{t}+5 \alpha u_{x}\right) d(u)+\left[\frac{1}{u_{x}} D-\frac{u_{x x}}{u_{x}^{2}}\right] D_{t}-\frac{1}{2} s_{1}^{a} D^{-1} \mathcal{C}_{1}+\frac{1}{2} u_{x} D^{-1} \mathcal{C}_{3}
\end{aligned}
$$

where

$$
\begin{aligned}
\mathcal{C}_{3} & =\left(\frac{u_{x t}}{u_{x}^{3}}-\frac{3 u_{t}-\alpha u_{x}}{2 u_{x}^{4}} u_{x x}\right) D^{2} \\
& +\left(-\frac{3 u_{x x} u_{x t}}{u_{x}^{4}}+3 \frac{4 u_{t}-\alpha u_{x}}{4 u_{x}^{5}} u_{x x}^{2}+\frac{3 \alpha u_{t}^{2}}{4}+\frac{3 \alpha^{2}}{2} u_{x} u_{t}+\frac{3 \alpha^{3}}{4} u_{x}^{2}\right) D \\
& -\frac{3}{4} u_{t} d(u)_{u} \\
& +\left[\frac{u_{x x}}{u_{x}^{3}} D-\frac{3 u_{x x}^{2}}{4 u_{x}^{4}}+\frac{3 u_{t}^{2}}{4}+\frac{3}{2} \alpha u_{t} u_{x}+\frac{3}{4} \alpha^{2} u_{x}^{2}-\frac{3 d(u)}{4}\right] D_{t}
\end{aligned}
$$

is the linearisation of the additional conserved density

$$
\rho_{3}=\frac{u_{x x} u_{x t}}{u_{x}^{3}}-\frac{3 u_{t}-\alpha u_{x}}{4 u_{x}^{4}} u_{x x}^{2}+\frac{u_{t}^{3}}{4}+\frac{3}{4} \alpha u_{t}^{2} u_{x}+\frac{3}{4} \alpha^{2} u_{t} u_{x}^{2}-\frac{3}{4} d(u) u_{t}+\frac{1}{4} \alpha^{3} u_{x}^{3} .
$$


The recursion operator $\mathcal{R}_{42}$ acts on symmetries as

$$
\begin{gathered}
\mathcal{R}_{42}\left(s_{i}^{a}\right)=s_{i+1}^{b} \\
\mathcal{R}_{42}\left(s_{j}^{b}\right)=s_{j+2}^{a}, i \geq 1, j \geq 0
\end{gathered}
$$

and Lemma 17 implies that $\mathcal{M}_{1}^{2}=\mathcal{L}_{2}^{3}$.

- The subcase with $d^{\prime}(q)=0$. This is a subcase of $\mathcal{L}_{5}$, and it admits a recursion operator $\mathcal{M}_{-1}$

$\mathcal{R}=\mathcal{M}_{-1}=-\frac{\alpha}{2} u_{x}-u_{t}+D^{-1} \cdot\left(\alpha u_{x}+\frac{1}{2} u_{t}\right) D+\left[\frac{1}{2} u_{x} D^{-1}+D^{-1} \cdot \frac{1}{2} u_{x}\right] D_{t}$.

that is a square root of $\mathcal{L}_{2}$ and generates a standard symmetry structure from $u_{t}$.

\subsubsection{Lagrangian $\mathcal{L} 5$}

This system admits the conserved densities $-\epsilon u_{x}^{2}-u_{x} u_{t}, \frac{\gamma^{2}}{u_{x}}+\gamma u_{t}$ with associated operators

$$
\mathcal{C}_{1}=\left(-2 \epsilon u_{x}-u_{t}\right) D-u_{x} D_{t}, \quad \mathcal{C}_{2}=-\frac{\gamma^{2}}{u_{x}^{2}} D+\gamma D_{t}
$$

A recursion operator is $\mathcal{M}_{-1}$

$$
\mathcal{R}_{5}=\mathcal{M}_{-1}=-\frac{\epsilon}{2} u_{x}-u_{t}-\frac{1}{2} D^{-1} \mathcal{C}_{1}+\frac{1}{2 \gamma} u_{x} D^{-1} \mathcal{C}_{2}
$$

Applied to $u_{t}$ this recursion operator generates the standard family of symmetries.

\subsubsection{Lagrangian $\mathcal{L} 6$}

There is a recursion operator $\mathcal{M}_{-1}$ :

$$
\mathcal{R}_{6}=\mathcal{M}_{-1}=\epsilon u_{x}-2 \epsilon D^{-1} \cdot u_{x x}-D^{-1} \cdot u_{x t}+D^{-1} \cdot u_{x} D_{t} .
$$

Applied to $u_{t}$ this recursion operator generates a standard family of symmetries.

\subsubsection{Lagrangian $\mathcal{L} 7$}

The equation with generic functions $a(u), b(u), d_{2}(u)$ and $d_{3}(u)$ admits a, notably, differential recursion operator

$$
\mathcal{R}_{71}=\mathcal{M}_{0}=-D \cdot \frac{u_{t}}{u_{x}}+D_{t}
$$


Applied to point symmetry $u_{x}$ the recursion operator yields 0 , i.e. $\mathcal{M}_{0}\left(u_{x}\right)=$ 0 . Applied to point symmetry $u_{t}$ it yields a higher symmetry

$$
\begin{aligned}
s_{2}^{4} & :=\frac{u_{x x x x}}{a^{4} u_{x}^{4}}-\frac{10 u_{x x} u_{x x x}}{a^{4} u_{x}^{5}}-\frac{8 a^{\prime} u_{x x x}}{a^{5} u_{x}^{3}}+\frac{2 b u_{x t}}{u_{x}}+\frac{15 u_{x x}^{3}}{a^{4} u_{x}^{6}}+\frac{24 a^{\prime} u_{x x}^{2}}{a^{5} u_{x}^{4}} \\
& -\frac{2 b u_{t} u_{x x}}{u_{x}^{2}}+b^{\prime} u_{t}-\frac{2\left(2 a a^{\prime \prime}+a^{6} d_{2}-10\left(a^{\prime}\right)^{2}\right) u_{x x}}{a^{6} u_{x}^{2}}+2 d_{2}^{\prime}+d_{3}^{\prime} u_{x} .
\end{aligned}
$$

This symmetry does not belong to one of the categorised symmetries, allowing the definition of a third class of symmetries

$$
\text { symmetries of type c: } s_{k}^{c}=e_{k} u_{2 k}+\cdots, \quad k=0,1, \ldots
$$

The recursion operator $\mathcal{M}_{0}$ generates an infinite hierarchy of higher symmetries of the type $s_{2 j}^{b}$, $s_{2 k}^{c}$ with $j \geq 1$ and $k \geq 2$.

This family of systems has subcases admitting more symmetries and recursion operators. We do not give here a full classification of subcases, but we show as an example the following system with a maximum number of independent symmetries:

$$
\mathcal{L}=\frac{u_{t}^{2}}{2 u_{x}}+\beta \frac{u}{u_{x}} u_{t}+\frac{u^{4}}{2 u_{x}^{5}} u_{x x}^{2}+\delta \frac{u^{2}}{u_{x}} .
$$

Some of its symmetries are $(\Delta:=\sqrt{8 \delta+9})$

$$
\begin{gathered}
u, \quad u_{x}, u_{t}, \frac{u_{x}}{u}, u^{-(1+\Delta) / 2} u_{x}, u^{-(1-\Delta) / 2} u_{x} \\
\frac{u^{2}}{u_{x}^{2}} u_{x x}, \frac{u}{u_{x}} u_{x t}-\frac{u u_{t}}{u_{x}^{2}} u_{x x} \\
\frac{u^{3} u_{x x} u_{x t}}{2 u_{x}^{4}}-\frac{u^{3} u_{t} u_{x x}^{2}}{2 u_{x}^{5}}-\frac{3 u^{2} u_{t} u_{x x}}{4 u_{x}^{3}}-\frac{\beta u_{t}^{2}}{2 u_{x}}+\frac{3 u u_{t}}{2 u_{x}}+\frac{\beta \Delta^{2} u^{2}}{8 u_{x}},
\end{gathered}
$$

All higher symmetries can be generated with $\mathcal{M}_{0}$ as in (6.2.1) and $\mathcal{L}_{1}$ i.e.

$$
\mathcal{R}_{71}=\mathcal{M}_{0}=-D \cdot \frac{u_{t}}{u_{x}}+D_{t}, \quad \mathcal{R}_{72}=\mathcal{L}_{1}=D \cdot \frac{u}{u_{x}} .
$$

\subsubsection{Lagrangian $\mathcal{L} 8$}

This system is equivalent to the Landau-Lifshitz model introduced in section 2.4 as a well know integrable Lagrangian system. It admits conserved densities

$$
\rho_{2}=\frac{u_{x} u_{t}}{u_{x}^{2}-1}, \quad \rho_{3}=\frac{u_{x x}^{2}-u_{t}^{2}}{2\left(u_{x}^{2}-1\right)}+d(u) u_{x}^{2}-\frac{1}{3} d(u)
$$

and $\mathcal{L}_{2}$ is a recursion operator

$$
\mathcal{R}_{81}=\mathcal{L}_{2}=D^{2}-\frac{u_{x} u_{x x}}{u_{x}^{2}-1} D+\frac{2}{3} d(u)+u_{t} D^{-1} \cdot \mathcal{C}_{2}-u_{x} D^{-1} \cdot \mathcal{C}_{3}
$$


where

$$
\mathcal{C}_{2}=-\frac{u_{x}^{2}+1}{\left(u_{x}^{2}-1\right)^{2}} u_{t} D+\frac{u_{x}}{u_{x}^{2}-1} D_{t}
$$

and

$$
\begin{aligned}
\mathcal{C}_{3}=\frac{u_{x x}}{u_{x}^{2}-1} D^{2}+\left[\frac{u_{t}^{2}-u_{x x}^{2}}{\left(u_{x}^{2}-1\right)^{2}} u_{x}+\right. & \left.2 d(u) u_{x}\right] D \\
& +\frac{1}{3}\left(3 u_{x}^{2}-1\right) d^{\prime}(u)-\frac{u_{t}}{u_{x}^{2}-1} D_{t} .
\end{aligned}
$$

The recursion operator $\mathcal{L}_{2}$ generates a standard symmetry hierarchy starting from seed symmetries $u_{x}$ and $u_{t}$.

The subcase with $d(u)=\delta=$ constant admits another first-order conserved density

$$
\rho_{1}=\frac{u_{t}}{1-u_{x}^{2}}
$$

that allows $\mathcal{M}_{-1}$ to be written in finite form as

$$
\mathcal{R}_{2}=\mathcal{M}_{-1}=D^{-1} \mathcal{C}_{1}-u_{x} D^{-1} \mathcal{C}_{2}
$$

with

$$
\mathcal{C}_{1}=\frac{2 u_{x} u_{t}}{\left(1-u_{x}^{2}\right)^{2}} D+\frac{1}{1-u_{x}^{2}} D_{t}
$$

In this subcase, the symmetry hierarchy can be generated by repeatedly applying $\mathcal{M}_{-1}$ to $u_{x}$. Lemma 17 implies that $\mathcal{R}_{1}=\mathcal{R}_{2}^{2}$. 

In this chapter we briefly review to which extent the initial objectives have been met, the way the research has been done, and in which ways there remain some open questions or the results obtained open new mattters of research. The objective of this thesis is to study the integrability (understood under the symmetry approach) of a generic family of equations arising as Euler-Lagrange equations of second order Lagrangian systems. As a first concrete novel result we then aimed to obtain a classification theorem for second order integrable Lagrangian systems.

The efforts to achieve this result started by studying the symmetry approach to integrability as detailed in chapter 1 , its theoretical foundations and computational techniques, how they are applied to the classification of integrable evolution equations and how the algebraic structure of the space of solutions to the linearized symmetry condition provides the basis for determining the integrability of a given equation. As the equations in our study are not evolution equations we found that the state of the art of the extensions of the symmetry approach should be elaborated further to obtain the required formulation for our purposes.

This led to the study of the structure of the space of FROs for the the $(4,1)$ case which in turn resulted in the determination of the precise structure for any pair of differential orders $(n, m)$, including the already known case $(3,1)$.

We can say, eventually, that the research required to resolve our initial problem resulted in a theoretical framework that can be applied to a much more wide class of equations.

\subsection{CONCLUSIONS}

At the same time, some interesting points arose during the research in this thesis.

- On the computational side, the work developed in this thesis required the development of computational techniques, based on the implementation of integrators for differential functions, that allow the study of non-homogeneous, non-polynomial and nonlinear equations.

- We have seen the convenience of the formulation in terms of recursion operators and in scalar notation to be computation- 
ally much easier and equivalent to working with symmetries or matrix recursion operators.

- All of the geometric properties of symmetries (from point to generalized as described in chapter I) forming algebraic groups and derived from the action of infinitesimal generators (which as fields represent the simplest form of differential operators) have their reflections in the recursion operator formalism employed to encode the integrability properties of equations. The main concept underlying this prolongation is the invariance along the solutions of certain objects encoded by the Lie derivative. These objects happen to be pseudo-differential operators acting as formal symmetries or formal recursion operators.

- As the most important achievement: the extension found to the symmetry approach, allowing the classification of very general non-evolutionary PDEs. Furthermore, this is valid or can be generalized to

- any differential order in the spatial variables,

- non-evolutionary equation with higher order in time derivatives.

\subsection{OPEN PROBLEMS}

We just highlight the most important, from those mentioned during this thesis, especially the computational aspects briefly described in chapter 4 .

- Integrable cases $\mathrm{L}_{4}$ and $\mathrm{L}_{7}$ have been only checked for some special values of the arbitrary functions in the Lagrangian. The exact form of the these we have the impression that could be derived from very high integrability conditions.

- The problem above is due to the high computational demand of the calculations for high rank densities.

- It would also be interesting to study the novelty of the integrable cases found, researching if there exist differential substitutions relating them to previously known integrable equations, or not.

- Extension of the theory to include some nonlocalities that, as it is known, allow the treatment of additional integrable cases. 
Part III

APPENDIX 



\section{CANONICAL DENSITIES}

We list here the first conservation laws $D_{t} \rho_{i}=D_{x} \sigma_{i}, i=0, . ., 7$ that constitute the integrability conditions ${ }^{1}$ for an equation of the form

$$
u_{t t}=F\left(u, u_{x}, u_{x x}, u_{x x x}, u_{x x x x} ; u_{t}, u_{x t}\right)
$$

where $a_{i}=\frac{\partial F}{\partial u_{i}}$ and $b_{i}=\frac{\partial F}{\partial u_{t i}}$.

$$
\begin{aligned}
& \rho_{0}=a_{4}^{-1 / 4}, \quad \rho_{1}=\frac{a_{3}}{a_{4}}, \quad \rho_{2}=\frac{b_{1}}{\sqrt{a_{4}}}-2 \frac{\sigma_{0}}{a_{4}^{1 / 4}} \\
& \rho_{3}=-\frac{3 b_{1} D_{x} a_{4}}{2 a_{4}^{5 / 4}}-\frac{2 \sigma_{1}}{a_{4}^{1 / 4}}-\frac{4 b_{0}}{a_{4}^{1 / 4}}+\frac{a_{3} b_{1}}{a_{4}^{5 / 4}} \\
& \rho_{4}=\frac{5\left(D_{x} a_{4}\right)^{2}}{a_{4}^{7 / 4}}-\frac{12 a_{3} D_{x} a_{4}}{a_{4}^{7 / 4}}-\frac{16 \sigma_{0}^{2}}{\sqrt[4]{a_{4}}}-\frac{16 \sigma_{2}}{\sqrt[4]{a_{4}}}-\frac{4 b_{1}^{2}}{a_{4}^{3 / 4}}-\frac{32 a_{2}}{a_{4}^{3 / 4}}+\frac{12 a_{3}^{2}}{a_{4}^{7 / 4}} \\
& \rho_{5}=\frac{5 S(0)\left(D_{x} a_{4}\right)^{2}}{a_{4}^{7 / 4}}-\frac{12 S(0) a_{3}\left(D_{x} a_{4}\right)}{a_{4}^{7 / 4}}-\frac{4 S(0) b_{1}^{2}}{a_{4}^{3 / 4}}-\frac{16 S(0)^{3}}{\sqrt[4]{a_{4}}}-\frac{16 S(2) S(0)}{\sqrt[4]{a_{4}}}-\frac{32 S(0) a_{2}}{a_{4}^{3 / 4}}+\frac{12 S(0) a_{3}^{2}}{a_{4}^{7 / 4}}-\frac{S(4)}{\sqrt[4]{a_{4}}} \\
& \rho_{6}=8 D_{t}\left(\frac{S(0)}{\sqrt[4]{a_{4}}}\right)+\frac{16 b_{1}\left(D_{x} S(0)\right)}{\sqrt[4]{a_{4}}}-\frac{3 S(0) b_{1}\left(D_{x} a_{4}\right)}{a_{4}^{5 / 4}}+\frac{3 b_{1}^{2}\left(D_{x} a_{4}\right)}{a_{4}^{3 / 2}}-\frac{3 a_{3}^{2}\left(D_{x} a_{4}\right)}{a_{4}^{5 / 2}}-\frac{2 a_{3}\left(D_{x} a_{4}\right)^{2}}{a_{4}^{5 / 2}}+\frac{4 a_{3}\left(D_{x x} a_{4}\right)}{a_{4}^{3 / 2}} \\
& +\frac{8 a_{2}\left(D_{x} a_{4}\right)}{a_{4}^{3 / 2}}+\frac{2 S(0) a_{3} b_{1}}{a_{4}^{5 / 4}}-\frac{8 S(0) b_{0}}{\sqrt[4]{a_{4}}}-\frac{4 S(0) S(1)}{\sqrt[4]{a_{4}}}-\frac{2 S(3)}{\sqrt[4]{a_{4}}}-\frac{2 a_{3} b_{1}^{2}}{a_{4}^{3 / 2}}+\frac{8 b_{0} b_{1}}{\sqrt{a_{4}}}+\frac{2 a_{3}^{3}}{a_{4}^{5 / 2}}-\frac{8 a_{2} a_{3}}{a_{4}^{3 / 2}}+\frac{16 a_{1}}{\sqrt{a_{4}}} \\
& \rho_{7}=-\frac{56 b_{1} a_{3}^{3}}{a_{4}^{11 / 4}}+\frac{128 S(0) a_{3}^{3}}{a_{4}^{5 / 2}}+\frac{96 b_{0} a_{3}^{2}}{a_{4}^{7 / 4}}+\frac{96\left(D_{x} S(0)\right) a_{3}^{2}}{a_{4}^{3 / 2}}+\frac{252 b_{1}\left(D_{x} a_{4}\right) a_{3}^{2}}{a_{4}^{11 / 4}}-\frac{192 S(0)\left(D_{x} a_{4}\right) a_{3}^{2}}{a_{4}^{5 / 2}}+\frac{48 S(1) a_{3}^{2}}{a_{4}^{7 / 4}} \\
& +\frac{40 b_{1}^{3} a_{3}}{a_{4}^{7 / 4}}-\frac{128 S(0) b_{1}^{2} a_{3}}{a_{4}^{3 / 2}}-\frac{182 b_{1}\left(D_{x} a_{4}\right)^{2} a_{3}}{a_{4}^{11 / 4}}-\frac{128 S(0)\left(D_{x} a_{4}\right)^{2} a_{3}}{a_{4}^{5 / 2}}+\frac{96 S(0)^{2} b_{1} a_{3}}{a_{4}^{5 / 4}}+\frac{32 S(2) b_{1} a_{3}}{a_{4}^{5 / 4}} \\
& +\frac{192 a_{2} b_{1} a_{3}}{a_{4}^{7 / 4}}-\frac{192 b_{1}\left(D_{x} a_{3}\right) a_{3}}{a_{4}^{7 / 4}}-\frac{384 b_{0}\left(D_{x} a_{4}\right) a_{3}}{a_{4}^{7 / 4}}-\frac{192\left(D_{x} S(0)\right)\left(D_{x} a_{4}\right) a_{3}}{a_{4}^{3 / 2}}-\frac{48 S(1)\left(D_{x} a_{4}\right) a_{3}}{a_{4}^{7 / 4}} \\
& -\frac{640\left(D_{x x} S(0)\right) a_{3}}{\sqrt{a_{4}}}+\frac{16 b_{1}\left(D_{x x} a_{4}\right) a_{3}}{a_{4}^{7 / 4}}+\frac{256 S(0)\left(D_{x x} a_{4}\right) a_{3}}{a_{4}^{3 / 2}}-\frac{512 S(0) a_{2} a_{3}}{a_{4}^{3 / 2}}-\frac{105 b_{1}\left(D_{x} a_{4}\right)^{3}}{a_{4}^{11 / 4}}-\frac{160 b_{0} b_{1}^{2}}{a_{4}^{3 / 4}} \\
& -\frac{16 S(1) b_{1}^{2}}{a_{4}^{3 / 4}}+\frac{280 b_{0}\left(D_{x} a_{4}\right)^{2}}{a_{4}^{7 / 4}}+\frac{20 S(1)\left(D_{x} a_{4}\right)^{2}}{a_{4}^{7 / 4}}-\frac{384 S(0)^{2} b_{0}}{\sqrt[4]{a_{4}}}-\frac{128 S(2) b_{0}}{\sqrt[4]{a_{4}}}-\frac{256 a_{2} b_{0}}{a_{4}^{3 / 4}}+\frac{512 S(0) b_{0} b_{1}}{\sqrt{a_{4}}} \\
& -\frac{256 a_{1} b_{1}}{a_{4}^{3 / 4}}-\frac{288 b_{1}^{2}\left(D_{x} S(0)\right)}{\sqrt{a_{4}}}+\frac{1024 S(0) b_{1}\left(D_{x} S(0)\right)}{\sqrt[4]{a_{4}}}-\frac{512 a_{2}\left(D_{x} S(0)\right)}{\sqrt{a_{4}}}+128 S(0)\left(D_{x} S(2)\right) \\
& +\frac{128 b_{1}\left(D_{x} a_{2}\right)}{a_{4}^{3 / 4}}+\frac{384 b_{0}\left(D_{x} a_{3}\right)}{a_{4}^{3 / 4}}-\frac{60 b_{1}^{3}\left(D_{x} a_{4}\right)}{a_{4}^{7 / 4}}+\frac{192 S(0) b_{1}^{2}\left(D_{x} a_{4}\right)}{a_{4}^{3 / 2}}-\frac{144 S(0)^{2} b_{1}\left(D_{x} a_{4}\right)}{a_{4}^{5 / 4}} \\
& -\frac{48 S(2) b_{1}\left(D_{x} a_{4}\right)}{a_{4}^{5 / 4}}-\frac{288 a_{2} b_{1}\left(D_{x} a_{4}\right)}{a_{4}^{7 / 4}}+\frac{112 b_{1}\left(D_{x} a_{3}\right)\left(D_{x} a_{4}\right)}{a_{4}^{7 / 4}}+\frac{512 S(0) a_{2}\left(D_{x} a_{4}\right)}{a_{4}^{3 / 2}} \\
& -96 D_{t}\left(\frac{a_{3}^{2}}{a_{4}^{7 / 4}}\right)+512 D_{t}\left(\frac{S(0)^{2}}{\sqrt[4]{a_{4}}}\right)+128 D_{t}\left(\frac{S(2)}{\sqrt[4]{a_{4}}}\right)+32 D_{t}\left(\frac{b_{1}^{2}}{a_{4}^{3 / 4}}\right) \\
& +160 D_{t}\left(\frac{a_{3}\left(D_{x} a_{4}\right)}{a_{4}^{7 / 4}}\right)-80 D_{t}\left(\frac{\left(D_{x} a_{4}\right)^{2}}{a_{4}^{7 / 4}}\right)+\frac{64 b_{1}\left(D_{x x} a_{3}\right)}{a_{4}^{3 / 4}}-\frac{320 b_{0}\left(D_{x x} a_{4}\right)}{a_{4}^{3 / 4}}+\frac{240 b_{1}\left(D_{x} a_{4}\right)\left(D_{x x} a_{4}\right)}{a_{4}^{7 / 4}} \\
& -\frac{160 b_{1}\left(D_{x x x} a_{4}\right)}{a_{4}^{3 / 4}}-\frac{192 S(0)^{2} S(1)}{\sqrt[4]{a_{4}}}-\frac{64 S(1) S(2)}{\sqrt[4]{a_{4}}}-\frac{64 S(0) S(3)}{\sqrt[4]{a_{4}}}-\frac{32 S(6)}{\sqrt[4]{a_{4}}}+\frac{1024 S(0) a_{1}}{\sqrt{a_{4}}}-\frac{128 S(1) a_{2}}{a_{4}^{3 / 4}}
\end{aligned}
$$

1 These conditions have been employed as the dependences encoding the integrability of the given equation as an input for the process of classification of second order integrable Lagrangian systems developed in this thesis. 

[1] V.E. Adler, A.B. Shabat, and R.I. Yamilov. "Symmetry approach to the integrability problem." In: Theoretical and Mathematical Physics 125, 3 (2000) (cit. on pp. 21, 22).

[2] Robert L. Anderson and Nail H. Ibragimov. Lie-Bäcklund Transformations in Applications. 1979. DoI: 10.1137/1.9781611970913 (cit. on p. xxii).

[3] A. V. Bäcklund. "Uber flachentransformationen." In: Math. Ann. IX (1876), pp. 297-320 (cit. on pp. xxi, xxii).

[4] G. I. Barenblatt. Scaling Phenomena in Fluid Mechanics. Cambridge University Press, 1995. URL: https : //Www . amazon. com/ScalingPhenomena - Mechanics - Isaakovich - Barenblatt/dp/0521469201? SubscriptionId=AKIAIOBINVZYXZQZ2U3A\&tag=chimboriO5 - 20\& linkCode $=x m 2 \&$ camp $=2025 \&$ creative $=165953 \&$ creativeASIN $=$ 0521469201 (cit. on p. xxii).

[5] A. E. Berriman. "The Babylonian Quadratic Equation." In: The Mathematical Gazette 40.333 (1956), p. 185. DOI: 10.2307/3608807 (cit. on p. xix).

[6] Luigi Bianchi. "Ricerche sulle superficie elicoidali e sulle superficie a curvatura costante." ita. In: Annali della Scuola Normale Superiore di Pisa - Classe di Scienze 2 (1879), pp. 285-341. URL: http://eudml .org/doc/82743 (cit. on p. xxi).

[7] Garrett Birkhoff. Hydrodynamics (Princeton Legacy Library). Princeton University Press, 1950. URL: https : / / www . amazon . com / Hydrodynamics - Princeton - Library - Garrett - Birkhoff / dp / 0691625913?SubscriptionId=AKIAIOBINVZYXZQZ2U3A\&tag=chimbori05$20 \&$ linkCode $=x m 2 \& \mathrm{camp}=2025 \& \mathrm{creative}=165953 \& \mathrm{creat}$ iveASIN $=$ 0691625913 (cit. on p. xxii).

[8] F. Calogero. Why Are Certain Nonlinear PDEs Both Widely Applicable and Integrable?, in the book V.E. Zakharov (ed.) What is Integrability? Springer Series in Nonlinear Dynamics. Springer, 1991 (cit. on p. 14).

[9] D. K. Demskoi and V. V. Sokolov. "On recursion operators for elliptic models." In: Nonlinearity 21.6 (2008), pp. 1253-1264. DOI: 10.1088/0951-7715/21/6/006 (cit. on p. 107).

[10] Gerhard Wanner Ernst Hairer. Analysis by Its History. Springer New York, Sept. 5, 2000. 392 pp. URL: https : / / www . ebook. de / de / product / 3242921 / ernst _ hairer _ gerhard _ wanner _ analysis_by_its_history.html (cit. on p. xix). 
[11] E. Fermi, P. Pasta, S. Ulam, and M. Tsingou. STUDIES OF THE NONLINEAR PROBLEMS. Tech. rep. 1955. DOI: 10.2172/4376203 (cit. on p. xxi).

[12] AS Fokas. "Symmetries and integrability." In: Studies in Applied Mathematics 77.3 (1987), pp. 253-299. DOI: 10.1002/sapm1987773253 (cit. on p. 10).

[13] G. Frobenius. "Ueber das Pfaffsche Problem." In: Journal für die reine und angewandte Mathematik (Crelle's Journal) 1877.82 (1877). DOI: $10.1515 /$ crll.1877.82.230 (cit. on p. xx).

[14] Clifford S. Gardner, John M. Greene, Martin D. Kruskal, and Robert M. Miura. "Method for Solving the Korteweg-deVries Equation." In: Physical Review Letters 19.19 (1967), pp. 1095-1097. DoI: 10.1103/phys revlett.19.1095 (cit. on p. xx).

[15] R. Hernández Heredero. Clasificación de ecuaciones de evolución integrables, Tesis Doctoral. Facultad de Ciencias Físicas, Universidad Complutense de Madrid, 1994 (cit. on pp. 15, 30, 72).

[16] R. Hernández Heredero, A.B. Shabat, and V.V. Sokolov. "A new Class of Linearizable Equations." In: Journal of Physics A (2003). URL: http : / / iopscience . iop . org / 0305 - 4470 / 36 / 47 / L02/ (cit. on pp. xxiv, 15, 38, 39, 44, 52, 72).

[17] R. Hernández Heredero, V.V. Sokolov, and S.I. Svinolupov. "Toward the classification of third order integrable evolution equations." In: Journal of Physics A (1994) (cit. on p. 15).

[18] N.Kh. Ibragimov and A.B. Shabat. "Evolutionary Equations with nontrivial Lie-Backlund Group." In: Ufa State University (1980) (cit. on p. 31).

[19] Nail H. Ibragimov and B. Kruglikov. Differential Equations : Geometry, Symmetries and Integrability : 159 The Abel Symposium 2008. Abel Symposia 5, DOI 10. 1007/978 - 3 - 642 - o0873 3 7. Springer - Verlag Berlin Heidelberg, 2009 (cit. on p. 5).

[20] Peter H. van der Kamp and Jan A. Sanders. "Almost integrable evolution equations." In: Selecta Mathematica 8 (2002), pp. 705719. DOI: 10.1007/bf02637315 (cit. on p. 10).

[21] D. J. Korteweg and G. de Vries. "XLI. On the change of form of long waves advancing in a rectangular canal, and on a new type of long stationary waves." In: The London, Edinburgh, and Dublin Philosophical Magazine and Journal of Science 39.240 (1895), pp. 422-443. DOI: 10. 1080/14786449508620739 (cit. on p. xx).

[22] Joseph-Louis Lagrange. Réflexions sur la résolution algébrique des équations. 1771. DOI: 10. 1017 / cbo9780511701795 . 008 (cit. on p. xix). 
[23] Peter D. Lax. "Integrals of nonlinear equations of evolution and solitary waves." In: Communications on Pure and Applied Mathematics 21.5 (1968), pp. 467-490. DOI: 10.1002 / сpa. 3160210503 (cit. on p. xxi).

[24] J. Liouville. "Note sur l'intégration des équations différentielles de la dynamique." In: Journal des matématiques pures et appliqués 19 (1855), pp. 251-283. DOI: 10.1007/bf02402878 (cit. on p. xx).

[25] A. V. Mikhailov, A. B. Shabat, and R. I. Yamilov. "The symmetry approach to classification of nonlinear equations. Complete lists of integrable systems." In: 42:4 (1987) (cit. on pp. xxiv, 37, 39).

[26] A.V. Mikhailov, A.B. Shabat, and V.V. Sokolov. The Symmetry Approach to Classification of Integrable Equations, in the book V. E. Zakharov (ed. ) What is Integrability? Springer Series in Nonlinear Dynamics, 1991 (cit. on pp. 4, 15, 25, 59).

[27] Alexander V. Mikhailov, Vladimir S. Novikov, and Jing Ping Wang. "On Classification of Integrable Nonevolutionary Equations." In: Studies in Applied Mathematics 118.4 (2007), pp. 419457. DOI: $10.1111 /$ j .1467-9590.2007.00376.x (cit. on p. 38).

[28] Robert M. Miura. "Korteweg-de Vries Equation and Generalizations. I. A Remarkable Explicit Nonlinear Transformation." In: 9 (1968), pp. 1202-1204. DOI: 10.1063/1. 1664700 (cit. on p. xxi).

[29] Robert M. Miura. "The Korteweg-de Vries Equation: A Survey of Results." In: SIAM Review 19.4 (1976), pp. vi-vi. DOI: 10.1137/1019101 (cit. on p. xxii).

[30] Robert M. Miura, Clifford S. Gardner, and Martin D. Kruskal. "Korteweg-de Vries Equation and Generalizations. II. Existence of Conservation Laws and Constants of Motion." In: Journal of Mathematical Physics 9.8 (1968), pp. 1204-1209. DoI: 10.1063/1. 1664701 (cit. on p. xxi).

[31] Juan J. Morales-Ruiz. "Picard-Vessiot theory and integrability." In: Journal of Geometry and Physics 87 (2015), pp. 314-343. DOI: 10.1016/j . geomphys .2014. 07.006 (cit. on pp. 8, 14, 15).

[32] Emmy Noether. Invariante Variationsprobleme. (see Transport Theory and Stat. Phys. 1 A971), 186-207 for an English translation). 1983. DOI: 10.1007/978-3-642-39990-9_13 (cit. on p. 10).

[33] Vladimir S. Novikov and Jing Ping Wang. "Symmetry Structure of Integrable Nonevolutionary Equations." In: Studies in Applied Mathematics 119.4 (2007), pp. 393-428. DoI: $10.1111 /$ j . 1467 9590.2007.00390.x (cit. on p. 38).

[34] P. J. Olver. Lie Group Applications to Differential Equations. Springer N. Y., 1993 (cit. on pp. 4-6, 8, 13, 15, 25).

[35] P. J. Olver. Equivalence, Invariants, and Simmetry. Cambridge Unniversity Press, 1995 (cit. on pp. xxii, 4, 8). 
[36] Peter J. Olver. "Evolution equations possessing infinitely many symmetries." In: Journal of Mathematical Physics 18.6 (1977), pp. 12121215. DOI: 10.1063/1.523393 (cit. on p. xxiii).

[37] Peter J. Olver. "Symmetry groups and group invariant solutions of partial differential equations." In: J. Differential Geom. 14 (1979), pp. 497-542. DOI: $10.4310 / j \mathrm{dg} / 1214435234$ (cit. on p. 7).

[38] L. V. Ovsiannikov. Invariant Solutions. 1982. DoI: 10.1016/b978๑- 12 - 531680-4.50010- 1 (cit. on p. xxii).

[39] Agustín Caparrós Quintero and Rafael Hernández Heredero. "Formal recursion operators of integrable nonevolutionary equations and Lagrangian systems." In: Journal of Physics A: Mathematical and Theoretical 51.38 (2018), p. 385201. DOI: $10.1088 /$ 1751-8121/aad63e (cit. on pp. xi, 40, 41, 50, 52, 53, 72).

[40] J. F. Ritt. Differential Algebra. AMS Coll. Publ. 33, New York, 1950 (cit. on p. 16).

[41] J. Scott Russell. "Report on waves." In: Fourteenth meeting of the British Association for the Advancement of Science 1 (1844), pp. 433435. DOI: 10.1017/s0370164600039754 (cit. on p. xxi).

[42] Jan A. Sanders and Jing Ping Wang. "Integrable systems and their recursion operators." In: Nonlinear Analysis: Theory, Methods E Applications 47.8 (2001), pp. 5213-5240. DOI: 10.1016 / s0362-546x (01)00630- 7 (cit. on p. 103).

[43] A.B. Shabat, V.E. Adler, V.G. Marikhin, A.A. Mikhailov, and V.V. Sokolov. Encyclopedia of integrable systems. Ed. by LD Landau Institute for Theoretical Physics. 2010 (cit. on p. 52).

[44] V. V. Sokolov. "Hamiltonian property of the Krichever Novikov equation." In: Sov.Math.Dokl. 30 (1984) 44761 (2006), pp. 165167. DoI: 10 . 1070 / rm2006v061n01abeh004302 (cit. on pp. 37, 107).

[45] V. V. Sokolov and A. B. Shabat. "Classification of integrable evolution equations." In: Mathematical physics reviews, Vol. 4. Vol. 4. Soviet Sci. Rev. Sect. C Math. Phys. Rev. Harwood Academic Publ., Chur, 1984, pp. 221-28o (cit. on pp. xx, 23).

[46] V.V. Sokolov. "On the symmetries of evolution equations." In: 43:5, 165-204 (1988) (cit. on p. 58).

[47] S. I. Svinolupov. "Second-order evolution equations with symmetry." In: Akademiya Nauk SSSR i Moskovskoe Matematicheskoe Obshchestvo. Uspekhi Matematicheskikh Nauk 40.5(245) (1985), pp. 263264 (cit. on pp. xxiii, 15, 30).

[48] S. I. Svinolupov and V. V. Sokolov. "On evolution equations with nontrivial conservation laws." In: 16, 317-319 (1982) (cit. on p. 30). 
[49] G. Taylor. "The Formation of a Blast Wave by a Very Intense Explosion. II. The Atomic Explosion of 1945." In: 201 (1950), pp. 175-186. DOI: 10.1098/rspa.1950.0050 (cit. on p. xxii).

[50] N. J. Zabusky and M. D. Kruskal. "Interaction of "Solitons" in a Collisionless Plasma and the Recurrence of Initial States." In: Physical Review Letters 15.6 (1965), pp. 240-243. Dor: 10.1103/ phys revlett. 15.240 (cit. on p. xxi).

[51] V. E. Zakharov and A. B. Shabat. "A scheme for integrating the nonlinear equations of mathematical physics by the method of the inverse scattering problem." In: Func. Anal. Appl. (1974), pp. 226-235. DOI: 10.1142/9789814317344_0045 (cit. on p. xxi). 



\section{INDEX}

allowed transformations, 55

C-integrability, 14

canonical conserved densities, 24

canonical densities, 24

characteristic, II

characteristic of a symmetry, 9

characteristic system, 5

commutator, 5

compatibility condition, 34

conservation laws, 22

conserved density, 23

contact conditions, 56

contact fields, 58

contact symmetries, 10

contact transformations, 14, 25, 56

continuous groups of transformations, 5

determining equations, 9

differential algebra, 16

differential consequence, 10

differential functions, II

differential operators, 11

differential substitutions, 55

Euler operator, 22

Euler-Lagrange equations, 22

evolution equations, 13

evolutionary fields, II

evolutionary symmetries, 14

evolutionary vector fields, II

exact solutions, 3

extended symmetry approach,

$$
41
$$

flux, 23

formal inverse of the total

derivative, 18

formal symmetry of rank k, 20
Fréchet derivative, 16

generalized symmetries, 10 generalized vector field, 10 geometric or point symmetries, 6

group, 4

high order or higher symmetries, 10

infinite formal series, 18 integrability conditions, 6 integrable, 15 integrable equations classes, 25

integrable systems, 6 invariants, 4

jet bundle, 6

Lagrangian density, 22

Lie algebra, 5

Lie derivative, 6

Lie's infinitesimal criterion of invariance, 5

local invariant, 5

Mathematica, 61

matrix formal recursion operator, 40

multiplication operator, 18

non-evolutionary systems, 38

normalization of FROs, 22

obstructions to integrability,

$$
25,27
$$

prolongation, 6 prolonged action, 7

prolonged vector fields, 7

pseudo-differential operators, 18

recursion operators, 10 
residue, 22

S-integrability, 14

separant, 59

symmetry, 3

symmetry approach, 37

symmetry conditions, 4 symmetry groups, 4

total derivative, 8

trivial conservation laws, 23

trivial symmetries, 12

variational derivative, 23

variational problem, 22 